\title{
Transient integral boundary layer method to simulate entrance flow conditions in quasi-one-dimensional arterial blood flow
}

\author{
Dissertation \\ zur Erlangung des Doktorgrades \\ der Mathematisch-Naturwissenschaftlichen Fakultäten \\ der Georg-August-Universität zu Göttingen
}

\author{
Vorgelegt von \\ Stefan Bernhard \\ aus Darmstadt \\ Göttingen 2006
}


D7

Referent: Prof. Dr. Andreas Tilgner Korreferent: Prof. Dr. Eberhard Bodenschatz Tag der mündlichen Prüfung: 


\section{Abstract}

Motivation: The pressure drop - flow relations in myocardial bridges and the assessment of vascular heart disease via fractional flow reserve (FFR) have motivated much research during the last decades. The aim of this study is to simulate several clinical conditions present in myocardial bridges to determine the flow reserve and consequently the clinical relevance of the disease. From a fluid mechanical point of view the pathophysiological situation in myocardial bridges involves entrance flow conditions in a time dependent flow geometry, caused by contracting cardiac muscles overlying an intramural segment of the coronary artery. These flows mostly involve flow separation and secondary motions, which are difficult to calculate and to analyse.

Methods: Since a three dimensional simulation of the haemodynamic conditions in myocardial bridges in a network of coronary arteries is time-consuming, we present a boundary layer model for the calculation of the pressure drop and flow separation. The approach is based on the assumption that the flow can be sufficiently well described by the interaction of an inviscid core and a viscous boundary layer. Under the assumption that the idealised flow through a constriction is given by near-equilibrium velocity profiles of the Falkner-Skan-Cooke (FSC) family, the evolution of the boundary layer is obtained by the simultaneous solution of the Falkner-Skan equation and the transient von-Kármán integral momentum equation.

Results: The model was used to investigate the relative importance of several physical parameters present in myocardial bridges. Results have been obtained for steady and unsteady flow through vessels with $0-85 \%$ diameter stenosis. We compare two clinical relevant cases of a myocardial bridge in the middle segment of the left anterior descending coronary artery (LAD). The pressure derived FFR of fixed and dynamic lesions has shown that the flow is less affected in the dynamic case, because the distal pressure partially recovers during reopening of the vessel in diastole. We have further calculated the wall shear stress (WSS) distributions in addition to the location and length of the flow reversal zones in dependence on the severity of the disease.

Conclusions: The described boundary layer method can be used to simulate frictional forces and wall shear stresses in the entrance region of vessels. Earlier models are supplemented by the viscous effects in a quasi three-dimensional vessel geometry with a prescribed wall motion. The results indicate that the translesional pressure drop and the mean FFR compares favourably to clinical findings in the literature. We have further shown that the mean FFR under the assumption of Hagen-Poiseuille flow is overestimated in developing flow conditions. 


\section{Contents}

Abstract ............................... iii

Table of Contents ........................ iv

Glossary . . . . . . . . . . . . . . . . .

Acknowledgments . . . . . . . . . . . . . . . . xi

Dedication . . . . . . . . . . . . . . . . . . xiii

1 Introduction 1

1.1 Historical review . . . . . . . . . . . . . . . . . . . . 1

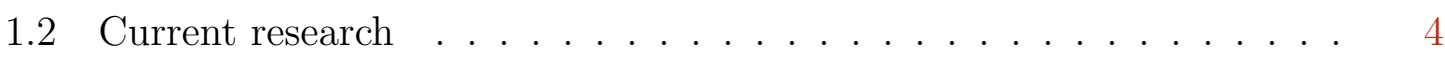

1.3 Objectives of this study . . . . . . . . . . . . . . 5

1.4 Thesis overview . . . . . . . . . . . . . . . . . 6

2 Model considerations $\quad 9$

2.1 The circulatory system . . . . . . . . . . . . . . . . . 9 9

2.1.1 The composition and properties of blood . . . . . . . . . 12

2.1.2 The structure and function of the heart . . . . . . . . . . 13

2.1.3 The systemic vasculature . . . . . . . . . . . . . . . . 15

2.2 Vascular pathology . . . . . . . . . . . . . . . . . . 16

2.2.1 Myocardial bridge . . . . . . . . . . . . . . . 17

2.3 Simplifications . . . . . . . . . . . . . . . . . . . 19

2.4 General fluid mechanical considerations . . . . . . . . . . . . . 20

2.4.1 Equations of motion . . . . . . . . . . . . . . . . 20

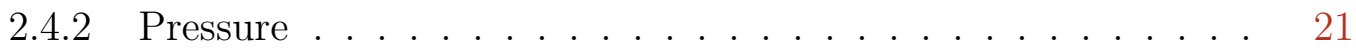

2.4.3 Flow rate . . . . . . . . . . . . . . . . . 21

2.4.4 Dimensionless parameters ............... 22

3 Averaged flow model $\quad 25$

3.1 Averaged flow equations . . . . . . . . . . . . . . . 26

3.2 Fluid structure interaction . . . . . . . . . . . . . . . . . . . . . 30

3.2.1 Geometrical model . . . . . . . . . . . . . . . . . 30

3.2.2 The pressure-area relationship for non-circular cross-sections . 32

3.2.3 Elastic modulus and wall thickness as a function of tube radius 36

3.3 Influence of viscosity . . . . . . . . . . . . . . . . . 40 
3.3.1 Laminar fully developed flow . . . . . . . . . . . . . . . . . 40

3.3.2 Oscillating pipe flow . . . . . . . . . . . . . . . . . . . 41

3.3 .3 Developing flow . . . . . . . . . . . . . . . . . . . . . . . 42

Entrance region . . . . . . . . . . . . . . . . . . . 43

Boundary layer equations . . . . . . . . . . . . . . . 44

Integral boundary layer equations . . . . . . . . . . . . . 46

Falkner-Skan equation . . . . . . . . . . . . . 47

Viscous friction and momentum correction . . . . . . . . . 49

Boundary layer separation . . . . . . . . . . . 50

3.4 Validity . . . . . . . . . . . . . . . . . . . . 52

4 Theoretical Aspects 53

4.1 Hyperbolicity and non-linearity . . . . . . . . . . . . . 53

4.2 Characteristic system . . . . . . . . . . . . . . . . 54

4.3 Interface and boundary conditions . . . . . . . . . . . . 56

4.3.1 Approximation of boundary characteristics . . . . . . . . 56

4.3.2 Inflow boundary conditions . . . . . . . . . . . 57

4.3 .3 Interface conditions . . . . . . . . . . . . . . . . . 58

4.3.4 Outflow boundary conditions . . . . . . . . . . . . 59

5 Numerical Aspects $\quad 61$

5.1 Choice of numerical method . . . . . . . . . . . . . 61

5.1.1 Discretisation of the averaged flow equations . . . . . . . 62

5.1.2 Discretisation of the von Kármán momentum equation . . . . 64

5.2 Numerical treatment of boundary conditions . . . . . . . . . . 66

5.2 .1 Inflow condition . . . . . . . . . . . . . . 67

5.2 .2 Bifurcations . . . . . . . . . . . . . . . 67

5.2 .3 Outflow condition . . . . . . . . . . . 67

6 Simulation Results $\quad 69$

6.1 Modelling: test geometry . . . . . . . . . . . . . . . . . . 70

6.1.1 Pressure drop and flow limitation . . . . . . . . . . 73

6.1.2 Separation and reattachment ............. . 74

6.1.3 Wall shear stress and friction coefficient . . . . . . . . . . 75

6.1.4 Unsteady solutions . . . . . . . . . . . . . . . 76

6.2 Modelling: physiological basis . . . . . . . . . . . . . 78

6.2.1 Mean pressure drop . . . . . . . . . . . . . . . 79

6.2.2 Pressure-flow relation . . . . . . . . . . . . . . . . . 79

6.2.3 Fractional flow reserve . . . . . . . . . . . . . . . 83

6.2.4 Influence of wall velocity . . . . . . . . . . . . . . 84

6.2.5 Wall shear stress oscillation ............... 85

6.3 Human arterial tree . . . . . . . . . . . . . . . . . . . 87 
6.4 Left coronary arteries . . . . . . . . . . . . . . . . . . . . . 89

6.4.1 Pressure notch . . . . . . . . . . . . . . . . . 89

6.4 .2 Vessel collapse and reopening . . . . . . . . . . . . . . . . . . 91

6.4.3 Flow velocity pattern . . . . . . . . . . . . . . . . . 91

6.4.4 Deformation time shift . . . . . . . . . . . . . 93

6.4.5 Segment length . . . . . . . . . . . . . . . . 93

6.4.6 Peripheral resistance . . . . . . . . . . . . . . . 93

7 Discussion, Conclusion and Outlook 95

7.1 Summary of the results . . . . . . . . . . . . . . . . . . 95

7.2 Discussion . . . . . . . . . . . . . . . . . . . . . 98

7.3 Conclusion . . . . . . . . . . . . . . . . . . . . . . . . 99

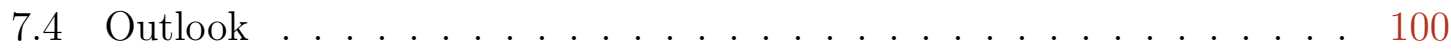

$\begin{array}{ll}\text { Bibliography } & 101\end{array}$

$\begin{array}{ll}\text { A Cardiovascular system parameters } & 113\end{array}$ 


\section{Glossary}

afterload

ametrohaemia

aneurysm

angina pectoris

angiography

anterior

ascending

atherosclerosis

atrium

bactericidal

cardio

catheter

coronary circulation

descending

diastole

disease

distal

epicardial

extramural

fractional flow reserve

haemodynamics

hyperaemia

intramural

invasive

in vitro

in vivo

ischaemia

lesion

lumen

microvasculature

myocardial bridge

myocardial contractility

myocardium

non-invasive

pathophysiology

perfusion pressure that the heart has to generate in order to eject blood

reduced blood circulation

local arterial widening

chest pain due to ischemia of the heart muscle

$\mathrm{X}$-Ray representation of the arteries

situated at the front

upward

arterial calcification or arterial plaque

upper chamber of the heart

substance that kills bacteria

medical term used to reference the heart

surgical instrument for invasive application

blood vessels that supply the myocardium

downward

relaxation of the heart muscle

illness

situated away from the median line of the body

on the outside of the cardiac muscle

outside the walls

fraction of normal flow to diseased flow

study of blood flow in the vasculature

increased blood circulation

inside the walls

medical application inside the body

in artificial conditions

in living organisms

restriction in blood supply

non-specific term referring to abnormal tissue

cavity or channel within a tubular structure

peripheral circulation

cyclic deformation of epicardial coronary arteries

intrinsic ability of a cardiac muscle fibre to contract

heart or cardiac muscle

medical application outside the body

study of conditions that cause diseases

delivery of arterial blood to a capillary bed 


$\begin{array}{ll}\text { phagocyte } & \text { eating cell } \\ \text { posterior } & \text { situated at the rear } \\ \text { proximal } & \text { situated towards the median line of the body } \\ \text { retrograde flow } & \text { back flow } \\ \text { revascularisation } & \text { to recover the blood circulation } \\ \text { stenosis } & \text { local arterial constriction } \\ \text { stent } & \text { arterial prosthesis, expandable wire form } \\ \text { systole } & \text { contraction of the heart muscle } \\ \text { translesional } & \text { across a lesion } \\ \text { transmural } & \text { across the walls } \\ \text { vasculature } & \text { network of arteries, capillaries and veins } \\ \text { ventricle } & \text { lower chamber of the heart } \\ \text { vessel } & \text { a tubular structure in anatomy, e.g. artery, vein } \\ \text { windkessel } & \text { elastic chamber into which the blood is pumped }\end{array}$




\section{Acknowledgments}

THIS dissertation was written in the department of Physics at the University of Göttingen. I thank all members of this department contributing to the completion of this work. I particularly note the sponsorship of the Georg-Christoph Lichtenberg foundation.

I would like to greatly thank my supervisor, Prof. Dr. Andreas Tilgner for every effort he made during this work, for his guidance, knowledge and patience. I also like to thank Prof. Dr. E. Bodenschatz for taking the part as second reviewer. Further, I like to thank Prof. Dr. med. F. A. Schöndube from the University Clinic of Göttingen, Prof. Dr. med. R. Erbel and PD Dr. med. S. Möhlenkamp from the West-German Heart Centre in Essen for their valuable contribution and comments to the present work.

I would like to thank the committee members Prof. Dr. Parlitz, Prof. Dr. Lauterborn, Prof. Dr. Glatzel, and PD. Dr. Rein for their suggestions and comments during the oral defence.

FURTHER, I like to thank my work group for the pleasant working atmosphere and Arno Ickler and Jürgen Ahrens for proof-reading the manuscript.

I am grateful to my parents, Wilfred and Brigitte, for their continued support and encouragement. Special thanks to my family, my wife Regina and my son Simon for their constant support, inspiration, and love. 
Dedicated to my Family

Die wahrhaft grosse Tradition in den Dingen liegt nicht darin, das nachzumachen, was die anderen gemacht haben, sondern vielmehr darin, den Geist wieder zu finden, der diese Dinge hervorgebracht hat und zu einer anderen Zeit ganz andere Dinge daraus erschaffen würde. - Paul Valery 


\section{Chapter 1}

\section{Introduction}

Tn this Chapter we give a short review about the role of the cardiovascular system,

1 historically and in current research. Further we point out the major objectives of this dissertation.

\subsection{Historical review}

THE quest for the understanding the processes occurring in the human arterial system reaches back to the $15^{\text {th }}$ century, when Leonardo da Vinci (1452 - 1519), a man infinitely curious and inventive, advanced the study of anatomy and developed insightful sketches of the anatomy of the heart and its blood vessels (see figure 1.1).

ONE hundred years later William Harvey (1578-1657) a medical doctor proposed pulsatile blood flow to be based on the periodic blood ejection of the heart combined with a continuous flow. Stephan Hales (1677-1761) was the first who measured the blood pressure in the arterial system and formulated the concept of peripheral resistance. In 1733 he discovered that the systemic arterial tree acts like an elastic chamber into which the blood is pumped. The pathology of phasic lumen obstruction of the coronary arteries by cardiac muscle fibres (myocardial bridging), was first mentioned in 1737 by H. C. Reyman in his dissertation "Disertatio de vasis cordis propriis" [1].

A simplified one-dimensional description of the human arterial system under normal physiologic conditions was introduced in 1775 by Leonhard Euler (1707-1783) a Swiss mathematician and physicist. He developed a system of non-linear partial differential equations, expressing the conservation of mass and momentum for inviscid flow. However, the wave nature of the arterial flow was first mentioned 1808 by Thomas Young in his publication about the constitutive equations for the behaviour of elastic walls under changes in transmural pressure. In 1878 Moens empirically determined the wave velocity in a thin-walled elastic tube containing an incompressible 

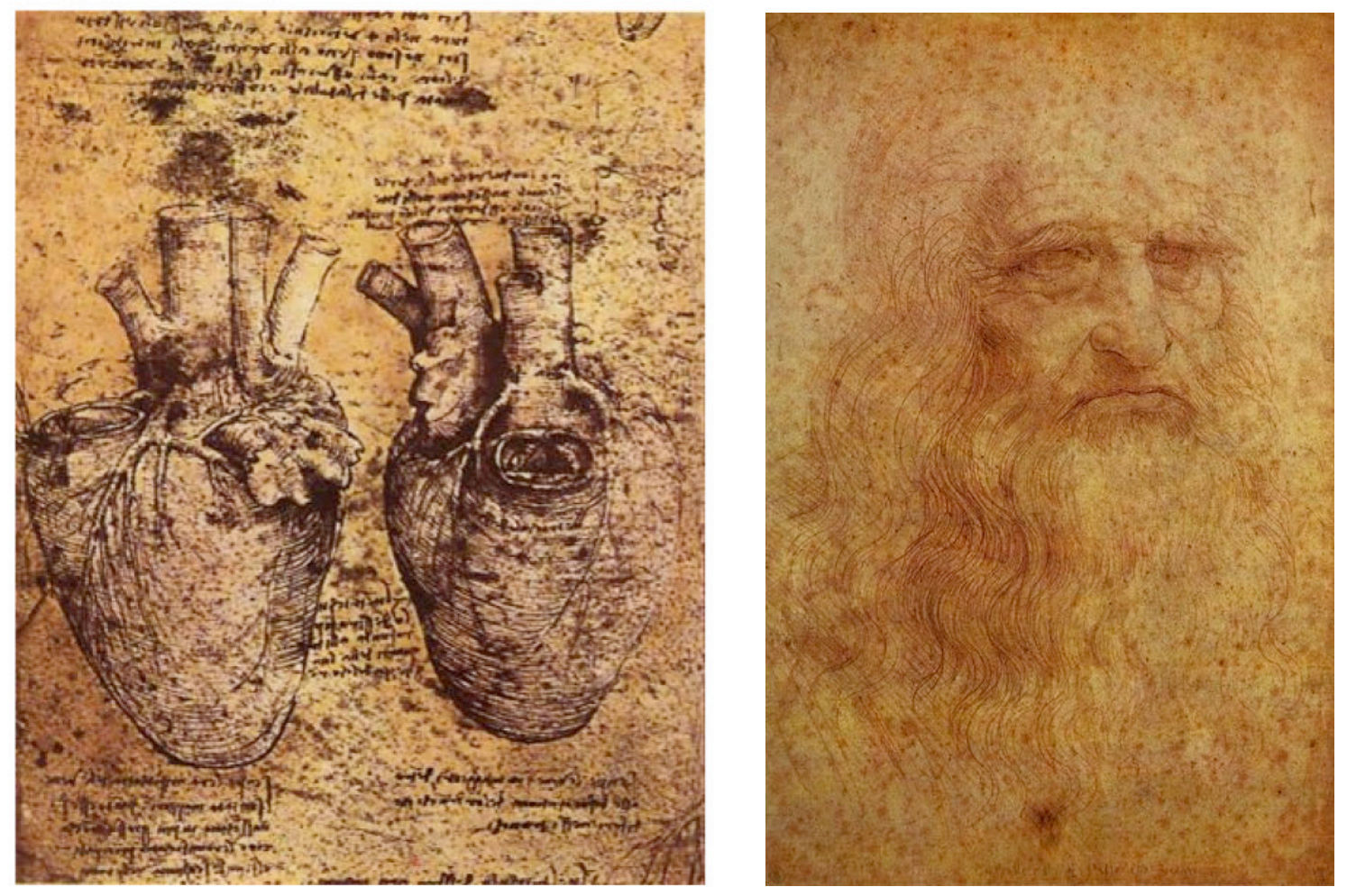

Figure 1.1: The understanding of the human heart 550 years ago. The sketches (left) of Leonardo da Vinci (right) show the heart and the distribution of the blood vessels.

non-viscous fluid. In the same year, Korteweg essentially determined the same function from a theoretical study of wave transmission. Today the expression is known as the Moens-Kortweg equation for the pulse wave velocity. In 1899 the idea of Hales was further modified by Otto Frank, who divided the pressure pulse into its diastolic and systolic components. Today his work is known as "windkessel" theory. It treats the aorta like an elastic tube with fluid storage capability, into which fluid is pumped at one end in an intermittent fashion, and flows out from the other end at an approximately constant rate. The circulatory system is thus conceived of as a central elastic reservoir into which the heart pumps blood, and from which the blood drains to the tissues.

THE mathematical analysis of the cardiovascular system was further advanced by the German mathematician Bernhard Riemann (1826-1866). He provided the method of characteristics, analytical tools to solve the system of hyperbolic equations proposed by Euler. At that time the major drawback of the one-dimensional description of blood flow was that viscous forces and the formation of flow profiles were not considered. The required progress was made by the French physician and physiologist Jean Poiseuille (1797-1869) who experimentally derived an expression for viscous flow 
in a circular tube. Together with Gotthilf Hagen (1797-1884) he formulated HagenPoiseuille's law (1838). The transition from laminar to turbulent flow was studied by the Irish fluid dynamics engineer Osborne Reynolds (1842-1912). In 1883 he proposed that a measure for the dynamic similarity of flows is given by the ratio of inertial forces to viscous forces, which he expressed in the dimensionless Reynolds number.

Although the adequate characterisation of viscous fluid motion in form of the Navier-Stokes equations (Claude-Louis Navier (1785-1836) and Gabriel Stokes (18191903)) was already known, the great mathematical difficulty of these equations hindered the treatment of viscous flow. The notion of a viscous boundary-layer was first developed by the German physicist Ludwig Prandtl (1875-1953) in the groundbreaking paper [2], "Über Flüssigkeitsbewegung bei sehr kleiner Reibung" in 1904. Using theoretical considerations together with experimental findings, he showed that the flow past a body can be divided into two regions: a very thin layer close to the body (boundary layer) where the viscosity is important, and the remaining region outside this layer where the viscosity can be neglected. A possible solution of these partial differential equations (boundary layer equations), for laminar flow over a flat plate, was given by $H$. Blasius in 1908. He applied a similarity transformation which turned the boundary layer equations into an ordinary differential equation for the stream function. Likewise a solution for laminar flow along a wedge was given by V. M. Falkner and S. W. Skan in 1931. They assumed that the inviscid outer flow follows a power law. In 1921 Theodore von Kármán (1881-1963) a student of Prandtl formulated an integral relation for the approximate solution of the boundary layer equations. The method was further modified by Veldman, who introduced a concept of strong viscous-inviscid interaction to model the pressure loss and the extent of the separation region after sudden changes in flow geometry.

AN important theoretical work concerning the two-dimensional velocity profiles in vessels was done by Womersley in a series of papers $[3,4,5,6]$, where he presented an analytic solution to the linearised, two-dimensional equations of oscillating pipe flow in a rigid, circular tube. He obtained a wave solution under the assumption of linear superposition of harmonic waves. The effects of viscosity were extensively studied by James Lighthill (1924-1998) a British mathematician who estimated the thickness of the boundary layer appearing in vessels by a linear theory [7] and found good agreement with the work of Womersley.

IN spite of many simplifications made by the one-dimensional description of wave propagation, the models are preeminently applicable to evaluate the dynamic behaviour of the cardiovascular system, last but not least for reasons that averaged flow variables are measurable. An overview reporting the concepts and results of the above methods are given in $[8,9,10,11,12,13,14,15,16]$. More recent studies concerning the one-dimensional theory are found in $[17,18,19,20,21,22,23,24,25,26]$. 


\subsection{Current research}

MANY investigators have made seminal contributions to the research of the cardiovascular system. The incomplete understanding of the pathophysiology and clinical relevance of myocardial bridges has been the subject of debate for the last quarter century. An overview of physiological relevant mechanisms of myocardial bridging, the current diagnostic tools and treatment strategies are found in $[27,28,29,30,31$, $32,33,34]$. Despite extensive studies on this subject there is no consensus on its clinical significance to myocardial ischaemia or angina pectoris.

FROM a medical point of view coronary angiography is limited in its ability to determine the physiologic significance of coronary stenosis [35, 36]. Therefore intracoronary physiologic measurement of myocardial fractional flow reserve (FFR) was introduced and has proven to be a reliable method for determining the functional severity of coronary stenosis. The FFR characterises the flow by the ratio of diseased and normal flow conditions. The assessment is independent of changes in systemic blood pressure, heart rate, or myocardial contractility and is highly reproducible [37]. Previous studies have shown that the cut-off value of 0.75 reliably detects ischaemia-producing lesions for patients with moderate epicardial coronary stenosis [38]. However, the flow-derived FFR is purely theoretic, so that the concept of pressure-derived FFR was introduced [39, 40, 41, 42] and clinically validated [43]. It was found to be very useful in identifying patients with multi-vessel disease [44], who might benefit from catheter-based treatment instead of surgical revascularisation.

To ascertain the severity of the disease it is often desirable to have simple models to predict the pressure drop and flow characteristics. The cardiovascular fluid dynamics presented in two and three-dimensional simulations mostly assumes stationary flow conditions $[45,46]$, because the setting of suitable boundary conditions for wave like phenomena is crucial. Frequently lumped parameter models are used to model specific outflow conditions. One of the major drawbacks of using detailed numerical solvers is the difficulty to incorporate moving walls and that only small regions of vasculature can be examined at a given time. However, the branch of one-dimensional flow-averaged models [23, 20, 47] is mostly concerned with normal physiologic conditions, because the assumption of fully developed flow profiles is insufficient for the calculation of pressure drop in stenoses. Several theoretical aspects of the general one-dimensional equations can be found in [48, 49, 50].

ANOTHER area in cardiovascular system modelling is the adequate description of the vessel wall as condition for pulse wave propagation. Several models have shown the relationship between diameter, compliance, stiffness or elastic modulus and the corresponding pressures and forces to which a blood vessel is subjected [51, 52, 53, 54]. Besides the influence that the vessel properties exhibit on the formation of the pulse contour, a strong dependency on the outflow condition of vascular networks is observed [55]. The outflow conditions are generally imposed by a "windkessel" model 
$[56,57,58]$ or by a structured tree outflow condition $[18,59]$. In this context the estimation of total arterial compliance and terminal resistance plays an important role $[60,61,62,63,64]$.

THE interesting dynamic phenomena of collapsible tubes, which may occur in certain physiologic flow conditions are discussed in [65, 66, 67, 68]. The non-linear coupling between the fluid pressure and tube wall can produce strong oscillations and conditions in which high-grade stenotic arteries may collapse [69]. We note that the compression of the artery in a myocardial bridge benefits the conditions for vessel collapse, so that flow limitation due to vessel closure becomes more likely with increasing deformation.

A variety of models concerned with arterial stenoses [70, 46, 71, 72] and series of stenoses [39, 73, 74] are found in the literature. Theoretical studies based on the boundary layer theory $[75,76]$ have been done to predict the location of maximum wall shear stress $[77,78,79]$ and the extent of flow separation located distal to fixed stenoses $[80,45,81]$. The process of flow separation and reattachment in indented channels was further discussed in [82, 80, 83, 84], whereas [85] pays attention to the flow in the entrance region and the flow profiles in branching tubes are discussed in[86]. Frictional losses in fixed stenoses are theoretically discussed in [70, 71], experimental studies are given in $[87,74]$. These models assume strictly circular cross-sections and mostly require tabulated coefficients. The review of the available literature reveals that only a few approximate methods exist which are able to predict the pressure drop (or friction factor) in non-circular ducts [88, 89, 90, 91, 92, 93].

THERE are a few models, which discuss flow in a time dependent two-dimensional flow geometry [94, 95, 96, 97]. These models assume rigid walls and are mainly focused on vortex formation and the wall shear stress distribution. However, [82] discussed the extent of the separation zone in a one-dimensional empirical parameter model using the concept of dividing streamline. They found good agreement with experiments in two-dimensional (partly) flexible indented channels [98]. A crosssection averaged flow model for time dependent vessel geometries as they appear in myocardial bridges was first given by [99]. This numerical model was advanced by a approximate boundary layer description to reproduce several important features of myocardial bridges, including adequate relations for the wall shear stress, frictional forces and conditions for flow separation in unsteady flow conditions [100].

\subsection{Objectives of this study}

BLOOD flow under normal physiologic and diseased conditions is an important field of study. The majority of deaths in the developed countries result from cardiovascular diseases, most cases are associated with some form of abnormal flow conditions in arteries. The complex anatomy of coronary vessels and developing flow conditions 
hindered the investigation of coronary haemodynamics. On this account theoretical models to predict the pressure-flow relations in myocardial bridges are not available. The objective of this dissertation is to investigate the nature of blood flow and to model the haemodynamic conditions in the coronary arteries to clarify recent doubts about the functional behaviour of myocardial bridges.

PRIOR studies of unsteady flow conditions indicate that the formation of velocity profiles in tubes is dependent on whether the flow is dominated by viscous or transient inertial forces. Clear distinction leads to simplifying assumptions, that the flow in one-dimensional flow averaged models [22, 20] is either approximated by steady flow conditions (Hagen-Poiseuille flow) or by oscillating pipe flow (Stokes layer). As a result of the former approach the velocity profile is parabolic and independent of time and axial position, i.e. the wall shear stress is prescribed, while in the latter approach the boundary layer thickness is dependent on the ratio between the frequency of oscillation and fluid viscosity. Even though both are valid for a specific type of flow, wrong estimates for the wall shear stress and viscous friction are likely found in intermediate flow situations, where the thickness of the viscous boundary layer and the Stokes layer are of the same order. In those situations neither viscous nor inertial forces can be justifiably neglected in the formation of the velocity profile.

RECENTLY [101] proposed a boundary layer model for oscillating arterial blood flow in large blood vessels, where the shape of the velocity profile depends on the axial pressure gradient. However, the assumptions made restrict the validity to transient dominated flows. In this study, however, we address developing flow conditions, which are frequently encountered in one-dimensional blood flow simulations. We propose a solution to the transient boundary layer equation, where the velocity profile function depends on axial position and time, so that we obtain adequate estimates for the wall shear stress and viscous friction. In contrast to other methods those values are dependent on both space and time. During this study we apply this method to simulate developing flow of an incompressible, viscous fluid in a network of elastic tubes in response to the aortic pressure. The tube characteristics and fluid properties are known, the developing flow conditions and the pressure response are desired quantities. The model is further used to simulate flow separation in a quasi threedimensional, time dependent stenoses geometry. With the aid of common tools for the assessment of flow limitation, we intend to investigate physiological relevant cases of blood flow through a myocardial bridge located in the middle segment of the left anterior descending coronary artery (LAD).

\subsection{Thesis overview}

THE dissertation is organised as follows: In this Chapter we have given a short review about the role of the cardiovascular system, historically and in current research. We have stated the major questions we intend to answer in this dissertation. 
IN Chapter 2 we take a look at the basic aspects of the cardiovascular system and the pathophysiology of myocardial bridges. The general laws of fluid mechanics involved in the Navier-Stokes equation are briefly discussed and useful simulation parameters and orders of magnitudes are given.

In Chapter 3, we derive the basic equations of haemodynamics, focusing on recent development in mathematical modelling of developing flow in the cardiovascular system. Starting from the Navier-Stokes equation we successively derive the onedimensional form of equations for averaged flow and the boundary layer equations with appropriate similarity solutions for developing flow under an external pressure gradient. We are further concerned with the description of the vessel deformation, the state equation for the vessel wall and fluid structure interaction (FSI).

In Chapter 4 we discuss the theoretical aspects and properties of the derived equation system and propose required boundary conditions for the solution of blood flow in a network of branching tubes.

CHAPTER 5 deals with the numerical treatment of the governing equations. The highly non-linear nature of the integral momentum equation and the non-linearity of boundary and interface conditions requires an efficient and stable method of solution. The numerical implementation utilising a MacCormack finite difference scheme [102, 103] with alternating direction is detailed. The method is quite simple, efficient, second order accurate in space and time and capable to capture the influence of space varying vessel properties such as tapering and local changes in geometry and the non-linearity of the equations.

IN Chapter 6 we present the numerical investigation of several physiological relevant cases of vessel deformation in the cardiovascular system. We first verify the validity of the modelling equations by presenting a set of results in a single tube. In this context we compare the results of stationary flow in a fixed environment with solutions in literature. Thereby the viscous friction coefficient serves as comparable quantity for the approximate solution from the boundary layer theory. Further, we have verified that the accumulated net flow caused by the compression of the artery is identical to the change in vessel volume caused by the compression. A standard application of the one-dimensional theory is the simulation of pressure and flow waves in the human arterial system. We present a qualitative numerical study of the changes of pressure and flow waves in the human vasculature in the presence of a myocardial bridge. Using classical tools like the pressure derived fractional flow reserve and the estimation of wall shear stress we deduce the influence of several physical parameters on the flow in the coronary arteries. In this context we examine a variety of haemodynamic conditions generally provoked by medical doctors with the aim to reveal the severity of the disease. Finally those results are compared with in vivo studies.

In the final Chapter 7 we discuss and conclude the results in reference to their convenience in medicine and we finish with suggestions for further work.

THE Appendix 7.4 contains several vascular parameters including the vessel size, 
elastic properties and their affiliations to bifurcations and outflow boundary conditions.

THE doctoral research has led to two publications, both articles on a methodology to simulate flow in a time dependent vessel geometry [99, 100] including frictional forces, flow separation and reattachment. The results presented in both publications can be found in chapter 6 , while the methodology in chapter 3 and 4 is given in the latter and former publication respectively. 


\section{Chapter 2}

\section{Model considerations}

$\mathrm{H}$ AEMODYNAMICS in the human circulatory system comprises four major issues: the physics of pressure and flow in vascular networks, the process of vascular autoregulation, its role in the living systems and the use of this knowledge for diagnostic and clinical activities. This chapter is concerned with fundamentals of the cardiovascular system and the physical principles of the mechanics of the circulation in large blood vessels. The governing constitutive equations are studied and the simulation parameters and orders of magnitudes are discussed.

\subsection{The circulatory system}

THE human circulatory system is divided into two main components: the cardiovascular system, and the lymphatic system. The cardiovascular system consists of the heart, which is a muscular pumping device that successively contracts to propel blood through a closed system of arteries, series of arterioles, capillary vessels and veins that interfuse the body. Arterioles are small arteries that deliver blood from larger arteries to the capillaries. Capillaries branch to form an extensive network throughout the tissue (capillary beds). The veins carry the blood back to the heart. The vessel diameter ranges from a few centimetres down to a few micrometers. The major components of the cardiovascular system are:

- blood: suspension of liquid plasma and cells

- heart: a muscular pump to move the blood

- vascular system: blood vessels (arteries, arterioles, capillaries, venules, veins) which carry blood to/from all tissues. 

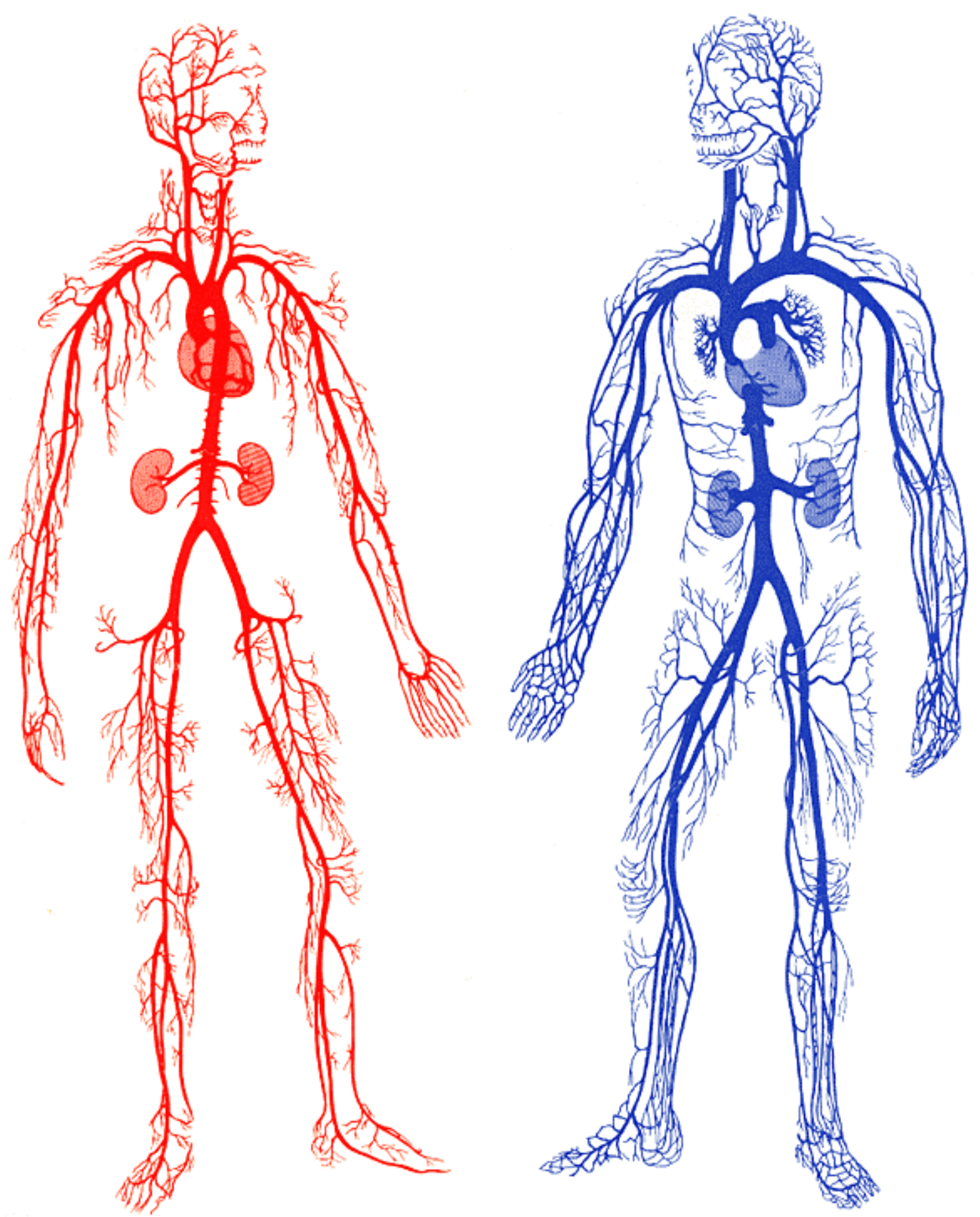

Figure 2.1: Schematic representation of the pulmonary and systemic circulatory systems. Vasculature carrying oxygenated blood (arterial system) is coloured red, while oxygen-poor blood (venous system) is coloured blue. 
THE primary function of the cardiovascular system is the transport of nutrient and gas throughout the body. The systemic circulation carries oxygenated blood from the left ventricle through the aorta to all parts of the body and returns oxygen-poor blood to the right atrium (auricle). Arteries distribute blood to a variety of organs and supply muscles with nutrition and oxygen, while the veins carry the blood back to the heart. A schematic representation of the pulmonary and systemic circulation is given in figure 2.1. A secondary function is the stabilisation of the body temperature and the $\mathrm{pH}$-value. The lymphatic circulation, collects fluid and cells and returns them to the cardiovascular system.

THE pulmonary circulation takes oxygen-poor blood from the right ventricle to the alveoli within the lungs and returns oxygenated blood to the left atrium. The alveoli are the final branchings of the respiratory tree and act as the primary gas exchange units of the lung, where oxygen diffuses in and carbon dioxide diffuses out. At the other end of the circulation microscopic blood vessels (the capillaries) are concerned with material exchange between the blood and tissue cells. The blood returns to the heart through the systemic veins. The capillaries unite to form series of venules which merge to form veins. All veins of the systemic circulation drain into the superior or inferior vena cava or the coronary sinus, which empty into the right atrium. The movement of blood along the veins is fundamentally different to that in arteries, because the pressure in veins is very low, so that nearby muscular contractions are required. To prevent back-flow of blood the veins are endowed with valves.

THIS highly sophisticated network of blood vessels and the auto-regulative mechanisms are optimised in such a way that all tissues are sufficiently well perfused in a number of different conditions. The responsibility therefor lies in the cardiovascular centre (CV), which is a group of neurons in the medulla oblongata that regulate the heart rate, its contractility and the blood vessel diameter. The CV receives input from higher brain regions and sensory receptors. Baroreceptors monitor blood pressure and chemoreceptors monitor blood levels of $\mathrm{O}_{2}, \mathrm{CO}_{2}$, and hydrogen ions. The distributing arteries have the ability to adjust the blood flow by vasoconstriction and vasodilation. Anyhow the arterioles posses the key role in regulating blood flow from arteries into the capillaries and in altering arterial blood pressure. The flow into the capillary beds is regulated by nerve-controlled sphincters. Under exercise for example the muscles desire more nutrients and oxygen, thus the heart rate increases and the flow resistance of the systemic vasculature is reduced.

THE auto-regulative mechanisms discussed in the previous passage are complex in their nature and a detailed representation is far beyond the scope of this study. However to take several haemodynamic conditions into account we adjust the parameters and boundary conditions accordingly. In the following sections we will discuss the functional behaviour of the major compartments and we simplify the system by common assumptions. Further we discuss the consequence of several pathologies. 


\subsubsection{The composition and properties of blood}

BLOOD is a suspension of shaped blood cells (coloured erythrocytes, colourless leukocytes and thrombocytes (see figure 2.2)) in an aqueous solution (the plasma). The properties of blood have several important functions. For example the ironcontaining haemoglobin in the erythrocytes causes vertebrate blood to turn red in the presence of oxygen; but more importantly haemoglobin molecules in blood cells carry oxygen to the tissues and collect the carbon dioxide. The blood also conveys nutritive substances like amino acids, sugars, mineral salts, hormones, enzymes and vitamins and it gathers waste materials which are filtered in the renal cortex of the kidneys. Furthermore it fulfils the defence of the organism by means of the bactericidal power of the blood serum, the phagocitic activity of the leukocytes and the immune response of the lymphocytes. The density, $\rho$, of blood at $37^{\circ} \mathrm{C}$ is $1055 \mathrm{~kg} / \mathrm{m}^{3}$, the total blood volume in an average adult is about 5 litres, or $8 \%$ of the total body weight. Formed elements constitute about $45 \%$ of the total blood volume, while the remaining $55 \%$ is blood plasma.

(a)

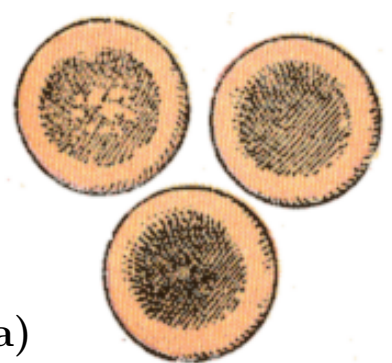

(b)

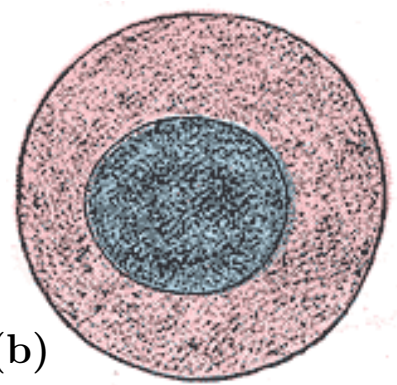

(c)

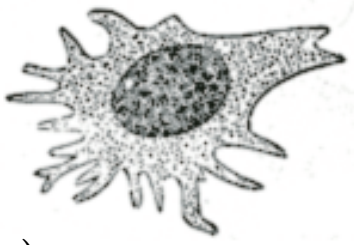

c)

Figure 2.2: The blood corpuscles: The erythrocytes (a), the leukocytes (b) and the thrombocytes (c). (Pictures are from the online version - "Anatomy of the Human Body" (1918), Henry Gray (1821-1865). Reprinted with kind permission from Bartleby.com, Inc.)

THE most important mechanical property of blood that influences its motion is the apparent viscosity $\mu$, which relates the shear rate $\gamma$ (velocity gradient) and the shear stress $\tau$. For the relationship in which the viscosity is independent of the shear rate the fluid is Newtonian and obeys Newton's law of viscosity.

$$
\mu=\frac{\tau}{\gamma}
$$

Especially in small vessels and arterioles where the shear rate is small blood is a non-Newtonian fluid. The rheology of non-Newtonian fluids is quite different to that of Newtonian fluids. The major difference is that the viscosity is dependent on the shear rate and that the stress relaxation and creep behaviour of the fluid is different. 
The viscosity of blood, mainly depends on the protein concentration of the plasma, the deformability of the blood cells, and the tendency of blood cells to aggregate [104]. With increasing shear rate, decreasing haematocrit or increasing temperature a reduction in blood viscosity is observed ${ }^{1}$. Experiments have shown that the nonNewtonian behaviour becomes insignificant when the shear rate exceeds $100 s^{-1}$ [70], a typical value in large vessels and that the apparent viscosity asymptotes to a value in the range of $3-4 \mathrm{mPas}$. In small vessels the shear rate is low and the viscosity rises steeply. For reasons that this dissertation is about the simulation of flow in large blood vessels, we consider blood to be an isotropic, homogeneous and incompressible Newtonian fluid.

\subsubsection{The structure and function of the heart}

THE human heart is the central organ of the cardiovascular system. It is a coneshaped, four-chambered structure of muscular walls (myocardium) and several valves. The right and left ventricle are separated by the inter-ventricular septum. The blood enters the heart in the atrium, the upper chamber and passes through the atrioventricular valve (AV) to enter the lower chamber, the ventricle. A semilunar valve (SL) separates each ventricle from its connecting artery.

THE cardiac cycle is generally subdivided into two phases, the systole (contraction of the heart muscle) and the diastole (relaxation of the heart muscle). The heart beat is controlled by the autonomic nervous system, which forces the contraction of the heart muscle by excitation of nodal tissue located in two regions of the heart. The sinoatrial node (SA node) initiates the heart beat while the atrioventricular node (AV node) causes the ventricles to contract. While the ventricles are contracted the atria are relaxed. These contractions occur regularly at the rate of about seventy per minute, projected to a normal lifetime this are over 3 billion contraction cycles. To satisfy increasing oxygen demands during exercise the major control variable is the heart rate, which can be varied by a factor of approximately 3, between 60 and 180 beats per minute, additionally the stroke volume is varied by a factor of 1.7 between 70 and $120 \mathrm{ml}$. The cardiac output (CO) is the volume of blood being pumped by the heart in one minute. It is equal to the heart rate multiplied by the stroke volume and lies between 4 and 30 litres/minute.

DURING the cardiac cycle the valves successively open and close to force the fluid flow into a single direction. Blood from the vena cava empties into the right atrium, at the same time oxygenated blood from the lungs flows from the pulmonary vein into the left atrium. The muscles of both atria contract, and the blood is forced downwards through the AV valves into the ventricles. Isovolumetric contraction of the ventricle ejects the blood, which is transmitted as a pulse wave along the arteries.

\footnotetext{
${ }^{1}$ In shear-thickening fluids the viscosity increases with shear rate, while in shear-thinning fluids the viscosity decreases with increasing shear rate.
} 
THE blood supply of the myocardium (cardiac muscle cells) is accomplished by a system of coronary arteries (see figure 2.3). The right and left coronary arteries branch from the aorta, the veins end in the right atrium. Under normal circumstances, coronary arteries have diameters large enough to transport sufficient amounts of oxygen to myocardial cells. Increases in myocardial oxygen demand, e.g. during exercise, are met by increases in coronary artery blood flow because - unlike in many other organs - extraction of oxygen from blood cannot be increased. This is in part mediated by increases in diameters of small intra-myocardial arteries. Yet, the large proximal (epicardial) coronary arteries contribute only a small fraction of total vascular resistance and show little variation in diameter during the cardiac cycle at any given metabolic steady state. For reasons that the epicardial coronary arteries are superficial the vessel curvature depends on the the actual position in the pulsatile cycle[105, 106]. Under maximum arteriolar vasodilation, the resistance imposed by the myocardial bed is minimal and blood flow is proportional to the driving pressure. In these conditions the flow in the coronary arteries is up to five times the normal flow. Blocked flow in the coronary arteries can cause ischaemia, and as a consequence disease of the heart muscle which finally leads to a heart attack.

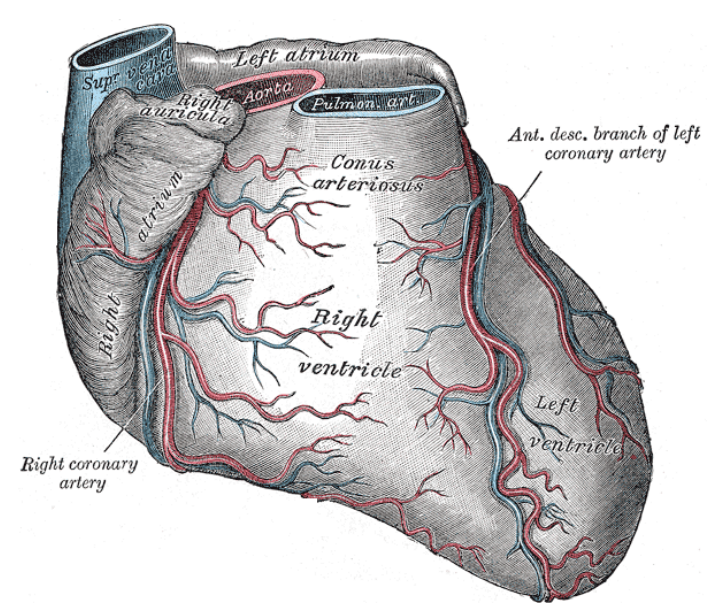

Figure 2.3: Front view of the heart and its connecting arteries.(Pictures are from the online version - "Anatomy of the Human Body" (1918), Henry Gray (1821-1865). Reprinted with kind permission from Bartleby.com, Inc.)

THE simulation of fluid flow in the heart is generally a three-dimensional attempt, thus our work is based on the assumption that the flow wave ejected from the heart is a sufficient inflow condition to the aorta and that no interaction takes place between the heart and the rest of the cardiovascular system, i.e. a given inflow is preserved despite the input impedance of the vascular system changes. 


\subsubsection{The systemic vasculature}

ESSENTIAL requirements in one-dimensional models of the arterial system is the adequate description of the arterial wall and the fluid structure interaction. One of the most important features of arterial walls is that they are able to expand and contract. This ability is a result of the mechanical properties of three composite layers in the arterial wall (see figure 2.4 (left)). The outermost layer comprises loose connective tissue (tunica externa), the middle layer consists of smooth muscle (tunica media) and the innermost layer is the endothelial tissue (tunica intima). The capillaries are composed of a single cell layer of endothelium. These elongated cells are generally aligned in the direction of flow and contribute negligibly to the mechanical properties of the artery. The major responsibility for its mechanical behaviour lies in the media. The smooth muscles in that layer have nearly circumferential orientation and the medial elastin allows elastic expansion under pressure, while the medial collagen prevents excessive dilation. The blood vessels must adapt to different physiologic demands and conditions from changes in blood pressure and flow. This response is typically governed by the need to control systemic vascular resistance. Activation of smooth muscles alters circumferential mechanical properties by constriction or dilation of the vessel.
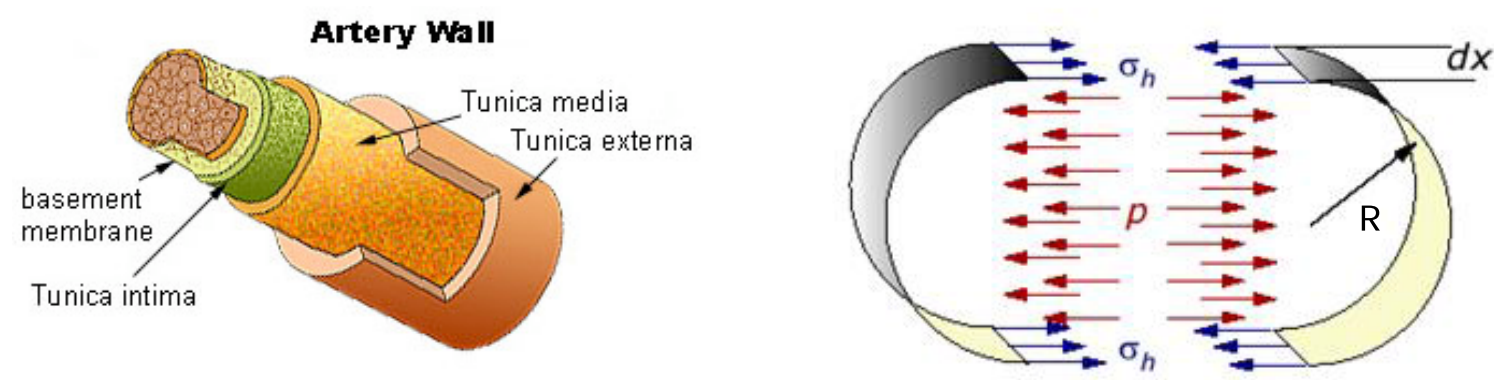

Figure 2.4: Structure of the arterial wall with distinct layers (left). Dilation of the vessel wall under pressure (right).

THERE are numerous studies of the mechanical properties of blood vessels $[107,104$, 108, 109, 52, 53, 54]. Experiments reveal that the mechanical behaviour of arteries becomes highly non-linear at high pressure and that the material is anisotropic ${ }^{2}$ and viscoelastic ${ }^{3}$ [111]. Furthermore the arterial wall is heterogeneous and tethered by surrounding tissue. Several studies have shown that incompressibility is a reasonable assumption, because biological tissues contain primarily water, which is incompressible at physiologic pressures. In healthy subjects, however, the properties of blood

\footnotetext{
${ }^{2}$ different properties in different directions

${ }^{3}$ exhibits creep, stress relaxation, and a hysteresis [110]
} 
vessels can be modelled as linear elastic, isotropic and homogeneous. Surrounding tissues and the muscular activity of the vessel are entirely disregarded in this study.

UNDER the assumption that the pressure in an axial section, $d x$, is constant (independent ring model) and that the wall displaces only in radial direction, the hoop stress, $\sigma_{h}$, in a thin-walled, linear elastic tube (see figure 2.4 (right)) can be estimated by Laplace's Law as

$$
\sigma_{h}=\frac{p R}{h_{0}}
$$

where $p$ is the excess pressure, $R$ is the inner radius of the vessel and $h_{0}$ is the wall thickness, which has to be small compared to the tube radius. As mentioned in the introduction the relationship between the pulse-wave velocity and the vessel and fluid properties for a linear elastic tube is given by the Moens-Korteweg equation

$$
c_{\text {pulse }}=\frac{1}{\sqrt{\rho_{0}\left(\frac{1}{\kappa}+\frac{2 R}{E h_{0}}\right)}},
$$

where $\kappa$ is the compressibility of the fluid and $E$ is the modulus of wall elasticity (Young's modulus). Whereas the speed of sound is mainly dependent on the compressibility of the fluid, the pulse wave velocity in elastic tubes is dependent on the distensibility of the tube. Increasing distensibility reduces the propagation speed of waves in the vasculature. Physiological relevant wave speeds are found in the range of $4-20 \mathrm{~m} / \mathrm{s}$, which is far below the speed of sound in blood ${ }^{4}$. The distensibility of large arteries like the aorta allows to accommodate the stroke volume of the heart during systole, while the elastic recoil during diastole provides pressure to propel the blood through small arteries and capillaries. The diameter and distensibility of arteries changes throughout the cardiac cycle to adapt the flow in certain conditions. Smaller arteries and arterioles are less elastic than larger arteries, and contain a thicker layer of smooth muscle. Their diameter is relative constant throughout the cardiac cycle.

\subsection{Vascular pathology}

MAINTENANCE and normal functioning of the circulatory system is fundamental for the human organism. Numerous pathological states may hinder the flow [87, 112] and reduced blood flow is the logical consequence. Pathological flow conditions in the coronary arteries are ominous for another reason; the muscle cells of the heart do not divide so that already dead muscle cells are not replaced. The blockage in the coronary arteries is usually a result of adhesion of cholesterol at the inner wall of the artery (atherosclerosis). Thereby the arterial lumen becomes locally restricted which is clinically referred to as a stenosis. The severity of stenoses is commonly defined as

\footnotetext{
${ }^{4} c_{\text {blood }} \approx 1500 \mathrm{~m} / \mathrm{s}$
} 
percent diameter occlusion. In contrast to atherosclerosis which forms over decades there are other congenital abnormalities which we discuss in further detail in this study.

EVIDENCE for the presence of stenoses in the coronary arteries emerge during periods of physical exercise, incidental chest pain and angina pectoris can occur if the oxygen demands under these conditions are not satisfied. Widely accepted indications of imminent heart attack are based on the presence of haemodynamically significant stenoses. The type of treatment is frequently selected by stenoses severity; the critical value for surgical treatment of arteriosclerosis in the left main coronary artery is at 50\% occlusion [113]. The morphology and consequently the percentage of the stenosis is generally obtained by using X-ray contrast angiography [36], information about the pressure drop, the flow rate, flow reserve, or location of the plaque can admittedly not be gained by this technique [35]. Experiments have shown that the pressure loss becomes significant in stenosis greater than 50-75\% [114, 115, 72, 74, 116] and depends on the length of the constriction and the Reynolds number. However, numerical studies based on patient specific data could provide approximate values for the pressure and flow rate in diseased conditions.

THE simulation of blood flow in diseased conditions of the coronary arteries is complicated for several reasons: Firstly, the left main coronary artery (LMCA) is short, so that the entrance region significantly affects the flow, secondly in comparison to most arteries the flow waveform in the left coronary artery is reversed [117] (having more flow during diastole) and thirdly the flow may be reversed during systole.

\subsubsection{Myocardial bridge}

IN contrast to temporal fixed stenoses, myocardial bridges are normal variants characterised by the compression of the coronary arteries due to myocardial muscle fibres overlying a segment of the artery. Myocardial bridges are most commonly found in the mid LAD, $1 \mathrm{~mm}$ to $10 \mathrm{~mm}$ below the surface of the myocardium with typical length of $10 \mathrm{~mm}$ to $30 \mathrm{~mm}$.

AN angiogram of a myocardial bridge is shown in figure 2.5 (A). The major characteristics of myocardial bridges are: a phasic systolic vessel compression with a persistent diastolic diameter reduction, increased blood flow velocities, retrograde flow, and a reduced flow reserve [31]. Within the bridged segments permanent diameter reductions of $22-58 \%$ were found during diastole, while in systole the diameters were reduced by $70-95 \%$ [31]. A schematic drawing of the vessel compression and the mean pressure tracings within the bridge are given in figure $2.5(\mathbf{B})$ and $(\mathbf{C})$ respectively. The underlying mechanisms are fourfold. Firstly the discontinuity causes wave reflections, secondly the dynamic reduction of the vessel diameter produces secondary flow and thirdly there is evidence for flow separation in post stenotic regions $[11,15,13]$. Finally at severe deformations the artery may temporally collapse. 


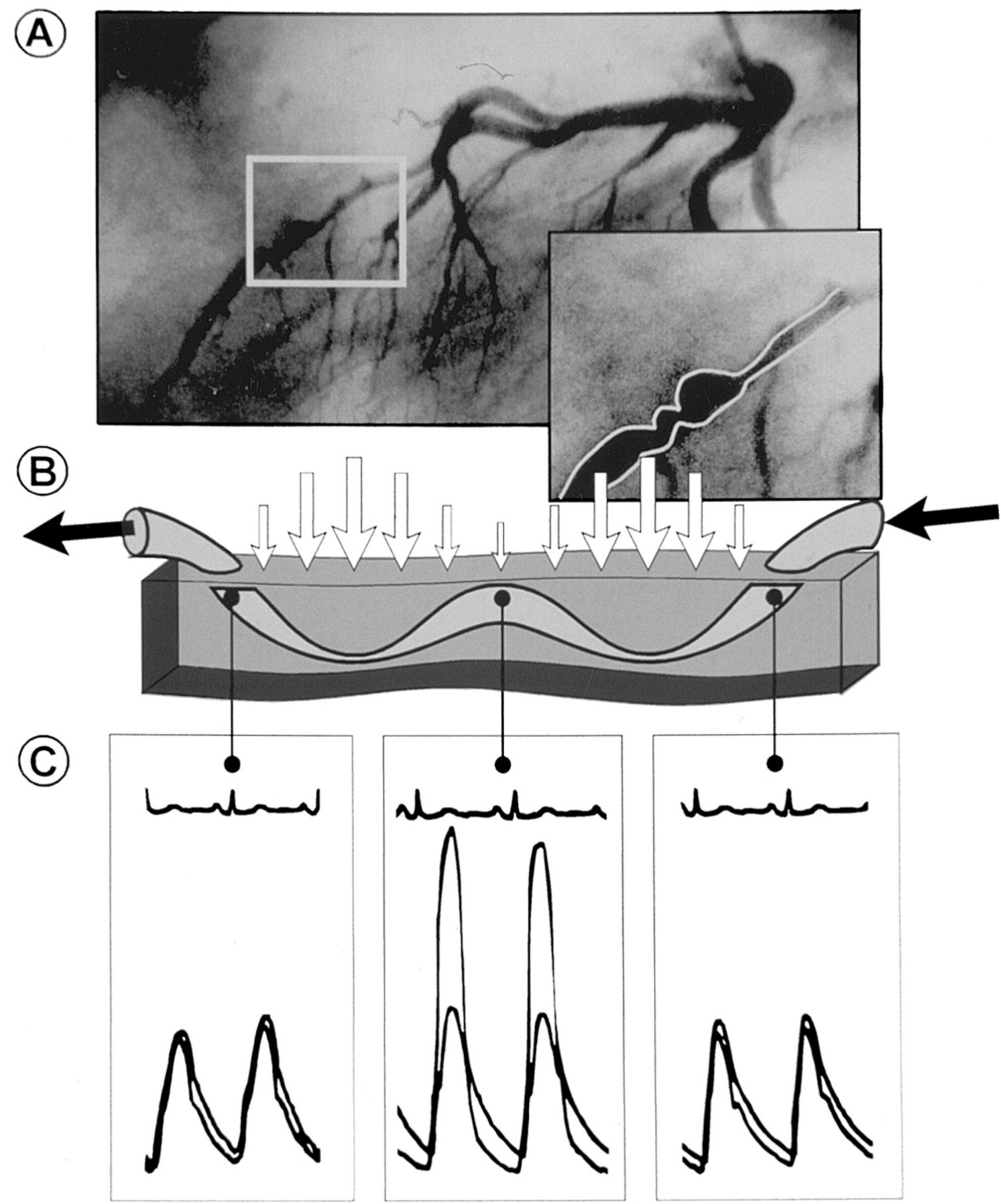

Figure 2.5: (A) Coronary angiogram of a myocardial bridge in the left anterior descending (LAD) branch in end systole. Compression of the artery during the heart's contraction phase, i.e. systole, is a characteristic finding in myocardial bridging (see text for details). (B) Semi-schematic drawing illustrates end systolic lumen dimensions and $(\mathbf{C})$ the measured pressure tracings proximal, within and distal the bridged segment. Picture taken from [31]. 


\subsection{Simplifications}

FACED towards the overwhelming complexity of the human cardiovascular system we summarise the main simplifications made in one-dimensional blood flow simulations. In contrast to unsteadiness, several features of biological flows may be neglected in some situations. A list of common assumptions is given below:

- blood is treated as incompressible Newtonian fluid

- flow is assumed to be laminar

- the heart is modelled by a volumetric inflow boundary condition

- axial curvature of the vessels is neglected

- arterial wall is assumed to be thin and incompressible

- non-linear behaviour of the arterial wall is not considered

- the effect of surrounding tissue and tethering is disregarded

- the muscular activity of the blood vessels is neglected

- only large arteries are accommodated in the network

- branching angles in the vascular network are neglected

- vessel geometry of the myocardial bridge is idealised

- flexural rigidity of the vessel wall is neglected

- deformation cross-section is simplified

- the arteriolar and capillary circulation is comprised into a "windkessel" boundary condition

- chemical reactions and diffusion of materials are not considered

- the auto-regulative feedback control is not considered

- haemodynamic conditions are adjusted by parameter control

Although each is physiologically relevant, the analysis is greatly simplified when these can be evidentiary neglected, which is the case in most arterial flows. In spite of many simplifications made, the models are pre-eminently applicable to evaluate the dynamic behaviour of the blood flow in the cardiovascular system. 


\subsection{General fluid mechanical considerations}

THE mathematical description of the fluid mechanics involved in the circulation is challenging in many aspects, even under the above simplifications. However, one way to study this complex system is to consider flow in isolated segments with appropriate boundary and interface conditions. One can then hope that this description of vascular networks can explain the characteristic pulsatile shapes of pressure and flow in different locations.

\subsubsection{Equations of motion}

THE equations of mass and momentum conservation fully describe the macroscopic behaviour of viscous flows. The equation of continuity states that the rate of mass accumulation is always equal to the difference between the rate of incoming and outgoing masses

$$
\frac{D \rho}{D t}+\rho(\nabla \cdot \vec{v})=0
$$

which for incompressible fluids simplifies to

$$
\nabla \cdot \vec{v}=0 .
$$

THE conservation of momentum follows from Newton's second principle: Mass per unit volume times acceleration is equal to the sum of three volume forces: the pressure force, the viscous force and the external force

$$
\rho \frac{D \vec{v}}{D t}=-\vec{\nabla} p-\vec{\nabla} \cdot \Gamma+\rho \vec{G} .
$$

In these equations, $D / D t$ is the substantial derivative, $\vec{v}$ is the velocity vector of the flow field, $p$ is the pressure, $\Gamma$ is the stress tensor and $\vec{G}$ is the external force. Assigning boundary conditions to the equations of motion yields a simplified set of equations, which is suitable for a specific type of flow problems. For example, the assumption of constant density and viscosity leads to the Navier-Stokes equations for incompressible Newtonian fluids

$$
\frac{D \vec{v}}{D t}=-\frac{1}{\rho} \vec{\nabla} p+\nu \vec{\nabla}^{2} \vec{v}+\vec{G}
$$

and for negligible viscous effects, $\vec{\nabla} \cdot \Gamma=0$, this reduces to the Euler equation

$$
\frac{D \vec{v}}{D t}=-\frac{1}{\rho} \vec{\nabla} p+\vec{G}
$$

IN this study we further assume that the external body force is zero, i.e. $\vec{G}=0$. The equations presented here are the fundamental equations used to derive the onedimensional model in chapter 3. In the following we briefly discuss the pressure and flow variables and their orders of magnitude. 


\subsubsection{Pressure}

In cardiovascular medicine the denotation of pressure is ambiguous. There is the transmural pressure, $p_{t m}$, which is the pressure difference between the inside $\left(p_{\text {int }}\right)$ and the outside $\left(p_{\text {ext }}\right)$ of a structure (ventricle, auricle or blood vessel) and the perfusion pressure, $p_{\text {perf }}$, which is the difference in transmural pressure between two different sites $\left(p_{t m 1}, p_{t m 2}\right)$ in the vascular network. Perfusion pressure is also called the driving pressure. Mean aortic pressure minus mean venous pressure yields the mean perfusion pressure, which is responsible for the blood flow through the systemic circulation from the aorta to the vena cava.

$$
\begin{gathered}
p_{t m}=p_{\text {int }}-p_{\text {ext }} \\
p_{\text {perf }}=p_{t m_{1}}-p_{t m_{2}}
\end{gathered}
$$

ThE blood pressure is generally measured in $\mathrm{mm}$ of mercury $(\mathrm{mmHg})^{5}$. Healthy young adults should have a systolic ventricular peak pressure of $16 \mathrm{kPa}(120 \mathrm{~mm} \mathrm{Hg})$, and a diastolic pressure of $10 \mathrm{kPa}(80 \mathrm{mmHg})$. The pressure distribution in the vasculature is shown in figure 2.6.

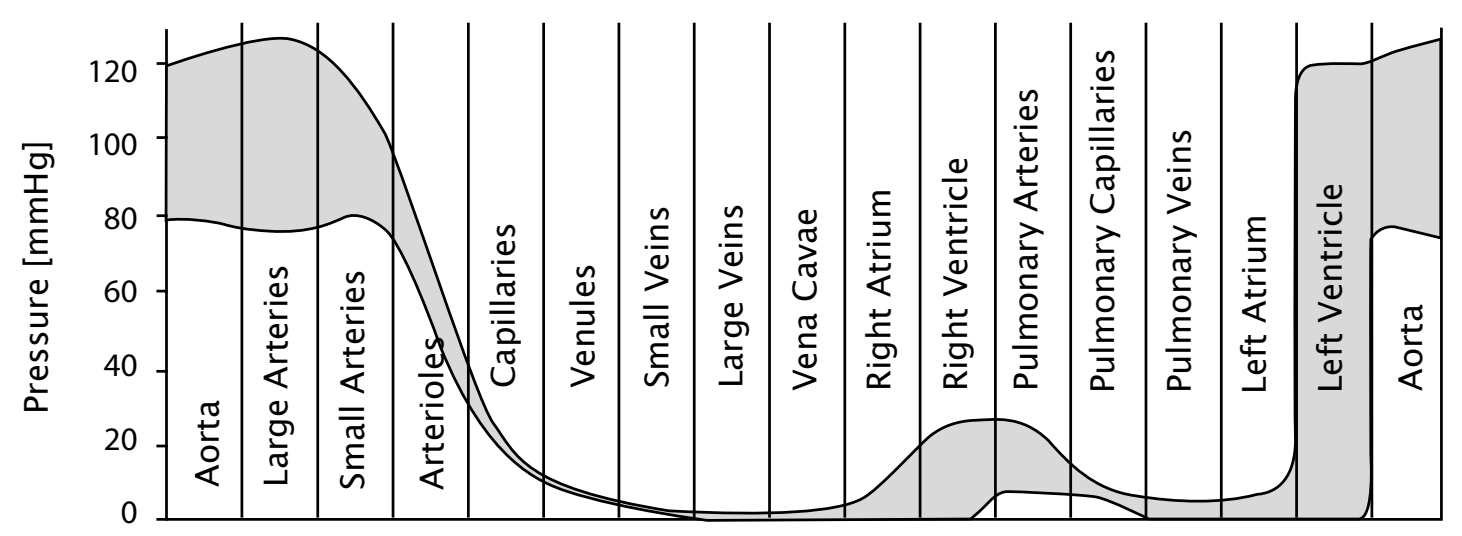

Figure 2.6: Pressure distribution in the human vascular system redrawn from [14].

\subsubsection{Flow rate}

As well as the pressure the flow rate, $q$, is patient specific. However, the aortic pressure and flow waveforms under certain conditions are known from in vivo measurements. In our simulations, the flow rate is a synthetic waveform represented as

\footnotetext{
${ }^{5}$ In Si-units 1 Torr $\equiv 1 \mathrm{mmHg} \equiv 133 \mathrm{~Pa}$.
} 
exponential pulse wave with parameters for the amplitude and raising time. Frequently, we also use a synthetic oscillatory waveform of a measured pulse wave to reproduce experimental data. In each case the shape of the flow rate will be shown together with simulation results produced by it.

\subsubsection{Dimensionless parameters}

IN order to simulate pulsatile flow in a model represented by segments of the cardiovascular system, a variety of similarity parameters characterise the flow behaviour in certain conditions. To investigate the importance of the pressure, viscous and transient forces, the following dimensionless numbers are defined:

- The Reynolds number indicates the relative importance of the viscous forces compared to the inertial forces. It is defined as

$$
R e=\frac{V \ell}{\nu},
$$

where $\ell$ is a characteristic length (such as the tube diameter), $\nu$ is the kinematic viscosity defined as $\nu=\mu / \rho$ and $V$ is the axial flow velocity ${ }^{6}$. Large Reynolds numbers indicate the dominance of convective inertia forces, while at low Reynolds numbers a strong influence of shear forces is observed. As already mentioned in the introduction the Reynolds number is a measure of turbulence. In circular tubes, three regions are recognised: laminar flow $(0<R e<2300)$, transient flow $(2300<R e<4000)$ and turbulent flow $(R e>4000)$. Since the geometry in the circulation is not regular, the characteristic length scale of the flow may also be defined as $\ell=\sqrt{A}$, where $A$ is the cross-section of the vessel. Under resting conditions the typical Reynolds numbers in the circulation are: $R e_{\sqrt{A}}=3800$ in the ascending aorta, $R e_{\sqrt{A}}=2800$ in the abdominal aorta, $R e_{\sqrt{A}}=450$ in the coronary arteries, and can be as small as ten in small arteries. We note that, due to the complex geometry of the arterial system instabilities like flow mixing, vortex formation, reverse flow and turbulent behaviour may occur even at low Reynolds numbers.

- The Womersley number is the ratio of the unsteady inertial forces to the shear forces. It is defined as

$$
W_{O}=\ell \sqrt{\frac{\omega}{\nu}}
$$

where $\omega$ is the angular frequency, which for a harmonic oscillation with period $T$ and frequency $f=1 / T$ is defined as $\omega=2 \pi f$. When the Womersley number

\footnotetext{
${ }^{6}$ The Reynolds number may be also defined in terms of the average flow velocity.
} 
is small, viscous forces dominate, and as a consequence the velocity profiles are parabolic in shape $[3,4,5]$. At larger Womersley numbers the unsteady inertial forces dominate, and the flow shows relative flat velocity profiles. Typical values under resting conditions are $W o_{\sqrt{A}}=33$ in the abdominal aorta and $W_{o_{\sqrt{A}}}=4$ in the coronary artery. As a consequence of increasing heart rate, the Womersley number increases under exercise.

- The Strouhal number represents a measure of the ratio of unsteady inertial forces to the inertial forces which follow from changes in velocity from one point to another in the flow field. The definition is

$$
S r=\frac{\omega \ell}{V}
$$

which can be rewritten as $S r=W o^{2} / R e$. It therefore combines the Reynolds and Womersley numbers. The Strouhal number is important for analysing unsteady, oscillating flow problems with moving walls. For small Strouhal numbers the influence of local acceleration on the flow is negligible so that the flow field can be assumed to be quasi-stationary, large values however require to specify the flow field in a temporal sense. Typical values in the circulation are $S r=0.15$ in the abdominal aorta and $S r=0.08$ in the coronary artery.

- The speed index is analogous to the Mach or Froude number. It is defined as the ratio of local flow velocity to local wave speed

$$
S=\frac{V}{c}
$$

where $c$ is the local wave speed. It determines the transition point from subcritical to supercritical flows. If the speed index $S$ is less than 1 the flow is subcritical, a value of $S>1$ indicates a supercritical flow.

THERE are numerous dimensionless parameters that are useful in fluid mechanics, however in this study we are concerned with the above mentioned. 


\title{
Chapter 3
}

\section{Averaged flow model}

\begin{abstract}
A variety of blood flow models are based on the Navier-Stokes equation or emanate from simplifying assumptions. The following chapter starts with the axisymmetric Navier-Stokes equation for incompressible fluids and discusses the orders of magnitude for several terms in a non-dimensional manner. By asymptotic reduction and subsequent integration over the cross-section the system of non-linear equations is expressed in averaged flow variables $(\mathrm{A}, \mathrm{u}, \mathrm{p})$. These equations are commonly accepted in quasi-one-dimensional models of the cardiovascular system [22, 23, 47, 18, 59, 118]. Essentially these models are based on five types of elements, namely, (i) quasi-onedimensional conduits, with lateral dimensions smaller than their length, (ii) locally restricted bifurcations, (iii) pathological segments (stenoses, aneurisms, stents, curved vessels), (iv) vascular termination (windkessel) and (v) aortic inflow condition. The main advantage of the quasi-one-dimensional formulation is that all important parameters can be identified by modern non-invasive measurement techniques, which is not the case for full three-dimensional models. The one-dimensional formulation, however, cannot resolve local blood flow patterns, e.g. flow and pressure distributions around stenoses or bifurcations, wall shear distributions, pressure drop or flow separation.
\end{abstract}

As mentioned in chapter 2 the flow in the left main coronary artery is of entrance type and the vessel deformation in myocardial bridges is a spatiotemporal event. Constitutive relation between the pressure and cross-sectional area in time varying non-circular ducts needs to be derived in order to calculate the flows under these conditions. The assumption of large Reynolds number flow allows the approximate solution to this type of flow via a boundary layer formulation. For this purpose the flow is divided into two regions, one close to the walls, where the flow is viscous (boundary layer) and the other in the centre where the flow is mainly inviscid (core flow). We subsequently derive the boundary layer equations for axially symmetric flows and the Falkner-Skan similarity solution in a vessel with spatiotemporal dependent pressure gradient. The evolution of the boundary layer is assumed to be 
quasi-stationary and the flow field is approximated by Hartree velocity profiles. Reasonable relations for viscous friction and momentum correction in the averaged flow equations are obtained from the von Kármán integral momentum equation. The chapter finishes with physiological conditions under which the considered model can give reasonable results.

\subsection{Averaged flow equations}

THE following derivation is motivated by [48], [49] and [50]. We consider the incompressible Navier-Stokes equations (2.7) in cylindrical coordinates $(x, r, \theta)$ with velocity components $\vec{v}(x, t)=\left(v_{x}(x, t, \theta), v_{r}(x, t, \theta), v_{\theta}(x, t, \theta)\right)^{T}$. We assume that all quantities are independent of the angular variable $\theta$, so that the equations reduce to

$$
\begin{aligned}
& \frac{\partial v_{x}}{\partial t}+v_{x} \frac{\partial v_{x}}{\partial x}+v_{r} \frac{\partial v_{x}}{\partial r}+\frac{1}{\rho} \frac{\partial p}{\partial x}=\nu\left[\frac{\partial^{2} v_{x}}{\partial r^{2}}+\frac{1}{r} \frac{\partial v_{x}}{\partial r}+\frac{\partial^{2} v_{x}}{\partial x^{2}}\right], \\
& \frac{\partial v_{r}}{\partial t}+v_{x} \frac{\partial v_{r}}{\partial x}+v_{r} \frac{\partial v_{r}}{\partial r}+\frac{1}{\rho} \frac{\partial p}{\partial x}=\nu\left[\frac{\partial^{2} v_{r}}{\partial r^{2}}+\frac{1}{r} \frac{\partial v_{r}}{\partial r}-\frac{v_{r}}{r^{2}}+\frac{\partial^{2} v_{r}}{\partial x^{2}}\right] .
\end{aligned}
$$

The continuity equation in cylindrical coordinates is given by

$$
\frac{\partial v_{x}}{\partial x}+\frac{1}{r} \frac{\partial}{\partial r}\left(r v_{r}\right)=0
$$

In flexible tubes the maximum value of the radial velocity, $v_{r}$, is that of the wall, which is generally much smaller than the axial flow velocity, $v_{x}$. The flow in the cardiovascular system is therefore essentially one-dimensional with its main flow direction along the axis of the tube. We define the dimensionless parameter

$$
\mathcal{E}:=\frac{\mathcal{V}_{r}}{\mathcal{V}_{x}}
$$

and the dimensionless variables

$$
r^{*}:=\frac{r}{\mathcal{R}}, \quad x^{*}:=\frac{x}{\mathcal{X}}, \quad v_{x}^{*}:=\frac{v_{x}}{\mathcal{V}_{x}}, \quad v_{r}^{*}:=\frac{v_{r}}{\mathcal{V}_{r}}, \quad p^{*}:=\frac{p}{\rho \mathcal{V}_{x}^{2}}, \quad t^{*}:=\frac{\mathcal{V}_{x}}{\mathcal{X}} t .
$$

For flow in large arteries like the aorta, carotid, or radial arteries the characteristic variables are typically

$$
\mathcal{V}_{x} \approx 0.5 \frac{\mathrm{m}}{\mathrm{s}}, \quad \mathcal{V}_{r} \approx 5 * 10^{-4} \frac{\mathrm{m}}{\mathrm{s}}, \quad \mathcal{R} \approx 10^{-3} \mathrm{~m}, \quad \nu \approx 4 * 10^{-6} \frac{\mathrm{m}^{2}}{\mathrm{~s}},
$$

so that the parameter $\mathcal{E}$ is of the order $\mathcal{O}\left(10^{-2}\right)$. From the continuity equation (3.2) it follows that $\mathcal{R} \mathcal{V}_{x}=\mathcal{X} \mathcal{V}_{r}$, thus $\mathcal{X}=\mathcal{R} / \mathcal{E} \approx 0.1 \mathrm{~m}$. Insertion of the dimensionless variables (3.4) into the continuity equation (3.2) yields

$$
\frac{\partial}{\partial x^{*}}\left(r^{*} v_{x}^{*}\right)+\frac{\partial}{\partial r^{*}}\left(r^{*} v_{r}^{*}\right)=0
$$


Expressing the first momentum equation (3.1a) in non-dimensional variables leads to

$$
\frac{\partial v_{x}^{*}}{\partial t^{*}}+v_{x}^{*} \frac{\partial v_{x}^{*}}{\partial x^{*}}+v_{r}^{*} \frac{\partial v_{x}^{*}}{\partial r^{*}}+\frac{\partial p^{*}}{\partial x^{*}}=\frac{1}{R e^{*}}\left[\frac{1}{\mathcal{E}} \frac{\partial^{2} v_{x}^{*}}{\partial r^{* 2}}+\frac{1}{\mathcal{E}} \frac{1}{r^{*}} \frac{\partial v_{x}^{*}}{\partial r^{*}}+\mathcal{E} \frac{\partial^{2} v_{x}^{*}}{\partial x^{* 2}}\right]
$$

where $R e^{*}$ is the Reynolds number defined by

$$
R e^{*}:=\frac{\mathcal{V}_{x} \mathcal{R}}{\nu},
$$

which for typical characteristic variables is of the order $\mathcal{O}\left(10^{2}\right)$. The preceding factors on the right hand side of equation (3.7) favour the negligence of the last term involving $\mathcal{E}$ compared with $\frac{1}{\mathcal{E}}$. Employing the continuity equation we obtain the first momentum equation in non-dimensional form

$$
\frac{\partial}{\partial t^{*}}\left(r^{*} v_{x}^{*}\right)+\frac{\partial}{\partial r^{*}}\left(r^{*} v_{x}^{*} v_{r}^{*}\right)+\frac{\partial}{\partial x^{*}}\left(r^{*} p^{*}\right)=\frac{1}{\mathcal{E} R e^{*}} \frac{\partial}{\partial r^{*}}\left(r^{*} \frac{\partial v_{x}^{*}}{\partial r^{*}}\right) .
$$

Inserting the non-dimensional variables into the second momentum equation (3.1b) gives

$$
\mathcal{E}^{2}\left[\frac{\partial v_{r}^{*}}{\partial t^{*}}+v_{x}^{*} \frac{\partial v_{r}^{*}}{\partial x^{*}}+v_{r}^{*} \frac{\partial v_{r}^{*}}{\partial r^{*}}\right]+\frac{\partial p^{*}}{\partial r^{*}}=\frac{\mathcal{E}}{R e^{*}}\left[\frac{\partial^{2} v_{r}^{*}}{\partial r^{* 2}}+\frac{1}{r^{*}} \frac{\partial v_{r}}{\partial r^{*}}-\frac{v_{r}}{r^{* 2}}+\mathcal{E}^{2} \frac{\partial^{2} v_{r}^{*}}{\partial x^{* 2}}\right]
$$

All terms except the pressure derivative on the left hand side of the above equation have a leading term of order $\mathcal{O}\left(10^{-4}\right)$, so that in a first approximation the pressure does not change in radial direction,

$$
\frac{\partial p^{*}}{\partial r^{*}}=0
$$

IN the following the dimensionless continuity and momentum equation are integrated over the cross-section from $r^{*}=0$ to $r^{*}=R^{*}$. The integration of equation (3.6) leads to

$$
\frac{\partial}{\partial x^{*}} \int_{0}^{R^{*}} v_{x}^{*} r^{*} d r^{*}-\left[v_{x}^{*} r^{*} \frac{\partial r^{*}}{\partial x^{*}}\right]_{r^{*}=R^{*}}+\left[v_{r}^{*} r^{*}\right]_{r^{*}=R^{*}}=0,
$$

and by the definition of the dimensionless mean flow in axial direction

$$
q^{*}:=2 \pi \int_{0}^{R^{*}} v_{x}^{*} r^{*} d r^{*}
$$

the continuity equation turns into

$$
\frac{1}{2 \pi} \frac{\partial q^{*}}{\partial x^{*}}-R^{*} \frac{\partial R^{*}}{\partial x^{*}}\left[v_{x}^{*}\right]_{r^{*}=R^{*}}+R^{*}\left[v_{r}^{*}\right]_{r^{*}=R^{*}}=0
$$


The integration of the first momentum equation (3.7) results in

$$
\begin{aligned}
\frac{\partial}{\partial t^{*}} \int_{0}^{R^{*}} v_{x}^{*} r^{*} d r^{*} & -\frac{\partial R^{*}}{\partial t^{*}}\left[v_{x}^{*} r^{*}\right]_{r^{*}=R^{*}}+\frac{\partial}{\partial x^{*}} \int_{0}^{R^{*}} v_{x}^{* 2} r^{*} d r^{*}-\frac{\partial R^{*}}{\partial x^{*}}\left[v_{x}^{* 2} r^{*}\right]_{r^{*}=R^{*}} \\
+ & {\left[v_{x}^{*} v_{r}^{*} r^{*}\right]_{r^{*}=R^{*}}+\frac{\partial p^{*}}{\partial x^{*}} \int_{0}^{R^{*}} r^{*} d r^{*}=\frac{1}{\mathcal{E} R e^{*}} \frac{\partial}{\partial r^{*}}\left[r^{*} \frac{\partial v_{x}^{*}}{\partial r^{*}}\right]_{r^{*}=R^{*}} . }
\end{aligned}
$$

The kinematic boundary condition and the non-slip condition at the arterial wall state that the radial fluid velocity at the wall is tangential to the surface

$$
\left[v_{r}^{*}\right]_{r^{*}=R^{*}}=\left[\frac{\partial r^{*}}{\partial t^{*}}\right]_{r^{*}=R^{*}}+\left[v_{x}^{*} \frac{\partial r^{*}}{\partial x^{*}}\right]_{r^{*}=R^{*}},
$$

and that the axial fluid velocity, $v_{x}$, at the wall is zero

$$
\left[v_{x}^{*}\right]_{r^{*}=R^{*}}=0
$$

Applied to the continuity equation this leads to

$$
2 \pi R^{*} \frac{\partial R^{*}}{\partial t^{*}}+\frac{\partial q^{*}}{\partial x^{*}}=0
$$

By defining another dimensionless parameter

$$
\chi^{*}:=\frac{2 \pi^{2} R^{* 2}}{q^{* 2}} \int_{0}^{R^{*}} r^{*} v_{x}^{* 2} d r^{*},
$$

and using equation (3.16) and (3.17), the momentum equation (3.15) may be written as

$$
\frac{\partial q^{*}}{\partial t^{*}}+\frac{\partial}{\partial x^{*}}\left(\chi^{*} \frac{q^{* 2}}{A^{*}}\right)+A^{*} \frac{\partial p^{*}}{\partial x^{*}}=\frac{1}{\mathcal{E} R e^{*}} \frac{\partial}{\partial r^{*}}\left[r^{*} \frac{\partial v_{x}^{*}}{\partial r^{*}}\right]_{r^{*}=R^{*}} .
$$

The coefficient $\chi^{*}$ is the ratio of momentum flux through a cross-section $A^{*}$ and the momentum flux with the average velocity through the same cross-section. It is constant if the velocity profile does not depend on $x$ and is equal to one if $v_{x}^{*}$ is independent of $r$ (uniform velocity profile). The most general form of the cross-section averaged equations finally is

$$
\begin{aligned}
\frac{\partial A^{*}}{\partial t^{*}}+\frac{\partial q^{*}}{\partial x^{*}} & =0, \\
\frac{\partial q^{*}}{\partial t^{*}}+\frac{\partial}{\partial x^{*}}\left(\chi^{*} \frac{q^{* 2}}{A^{*}}\right)+A^{*} \frac{\partial p^{*}}{\partial x^{*}} & =\frac{1}{\mathcal{E} R e^{*}} \frac{\partial}{\partial r^{*}}\left[r^{*} \frac{\partial v_{x}^{*}}{\partial r^{*}}\right]_{r^{*}=R^{*}} .
\end{aligned}
$$

These equations may be rewritten in terms of dimensional quantities through the definition of a dimensional volume flow

$$
q:=2 \pi \int_{0}^{R} v_{x} r d r
$$


which by definition leads to the expression

$$
q^{*}=\frac{q}{\mathcal{V}_{x} \mathcal{R}}
$$

Finally the dimensional form of the one-dimensional equations for blood flow in arteries are

$$
\begin{aligned}
\frac{\partial A}{\partial t}+\frac{\partial q}{\partial x} & =0 \\
\frac{\partial q}{\partial t}+\left[\frac{\partial}{\partial x}\left(\chi \frac{q^{2}}{A}\right)+\frac{A}{\rho_{0}} \frac{\partial p}{\partial x}\right] & =F_{\nu}
\end{aligned}
$$

where the momentum correction, $\chi$, is defined as

$$
\chi:=\frac{2 \pi}{A u^{2}} \int_{0}^{R} v_{x}^{2} r d r
$$

and the viscous friction term

$$
F_{\nu}:=2 \pi \nu R\left[\frac{\partial v_{x}}{\partial r}\right]_{r=R} .
$$

We finally rearrange the equations written in area and flow rate in terms of area and area-averaged axial flow velocity so that

$$
\begin{aligned}
\frac{\partial A^{\prime}}{\partial t} & =-\left[\frac{\partial A u}{\partial x}+\frac{\partial A_{d}}{\partial t}\right] \\
\frac{\partial u}{\partial t} & =-\left[\frac{u(\chi-1)}{A} \frac{\partial A u}{\partial x}+u \frac{\partial \chi u}{\partial x}+\frac{1}{\rho_{0}} \frac{\partial p}{\partial x}\right]+\frac{F_{\nu}}{A} .
\end{aligned}
$$

We have assumed that the total cross-sectional area is given by the equilibrium crosssectional area $A_{d}$ and the pressure induced perturbation $A^{\prime}$, so that $A=A_{d}+A^{\prime}$. The derivative of $A_{d}$ with respect to time on the right hand-side of equation (3.27a) is a prescribed function depending on the contraction of the cardiac muscles overlying the segment of the artery and is responsible for secondary fluid motion.

Throughout this study we mainly discuss flow in specific arterial networks, so that the dimensional form of the equations is used. Closer attention reveals that the two equations posses three unknowns $(\mathrm{A}, \mathrm{u}, \mathrm{p})$. To find a unique solution a third equation relating the pressure and cross-sectional area is required. This constitutive equation is the subject of the next Section. 


\subsection{Fluid structure interaction}

As previously discussed arterial walls possess complex elastic behaviour, if the fluid is driven by an oscillating pressure gradient. Negligence of material nonlinearities and viscoelasticity simplifies the description, but nonetheless the equations are geometrically nonlinear - the diameter of the vessel varies with the pulsating pressure. In large vessels, such as the aorta, the carotid or the radial arteries, a maximum change of $10 \%$ in the vessel diameter is expected. This results in the same change in the Womersley and Reynolds numbers. Even though a linearisation about an equilibrium cross-section is possible under these conditions, the cyclic deformation of the arterial wall in myocardial bridges is geometrically nonlinear and we dismiss this simplifying assumption.

THIS section starts from a simplified geometry found in most myocardial bridges [119] and subsequently derives an analytical expression for the relation between the pressure and area of a non-circular tube. Finally the elastic properties and the wall thickness of vessels are related to their diameter by experimental findings.

\subsubsection{Geometrical model}

OuR first geometrical simplification for modelling blood flow in arteries is that the axial curvature of the tube is assumed to be small everywhere, so that the problem can be defined in one space dimension along the $x$-axis. According to this we have simplified the anatomy of the myocardial bridge as shown in figure 3.1.

THE two arrows in figure 3.1 denote the location of either circular $(B-B)$ or oval $(C-C)$ cross-section of the tube. Due to the fact that the wall thickness, $h_{0}$, is small compared to the bending radius $R_{d}\left(h_{0} / R_{d} \ll 1\right)$, we assume that the bending stress inside the wall is negligible. Consequently the cross-section of a circular tube (figure 3.2 (left)) under deformation in $z$-direction, is given by the composition of a rectangle with two semicircular ends of bending radius $R_{d}$ as illustrated in figure 3.2 (right). This is consistent with the predominately eccentric deformation of bridged segments found in [119, 29]. We note that negligence of bending stress causes the tube to collapse significantly earlier, i.e. the assumption is only satisfied if $p_{t m} \geq 0$.

THE deformation distance between the squeezing muscles and the width of the flat portion are denoted by $D(x)$ and $B(x, t)$ respectively. The equilibrium condition of the cylindrical tube figure 3.2 (left) is denoted by the inner radius, $R_{0}$, the circumference $U_{0}=2 \pi R_{0}$ and the cross-section $A_{0}=\pi R_{0}^{2}$. However, the equilibrium cross-sectional area of the deformed tube is $A_{d}(x, t)$ (see figure 3.2 (right)). The total cross-sectional area in the $y z$-plane of the tube is defined by $A(x, t)=\int_{A} d a$ and the actual circumference is $U_{p}(x, t)$. Consequently the average velocity is given by $u(x, t)=\frac{1}{A} \int_{S} \vec{v}_{x} d a$ and the volume flux across a given section therefore is $q(x, t)=$ $A u$. 


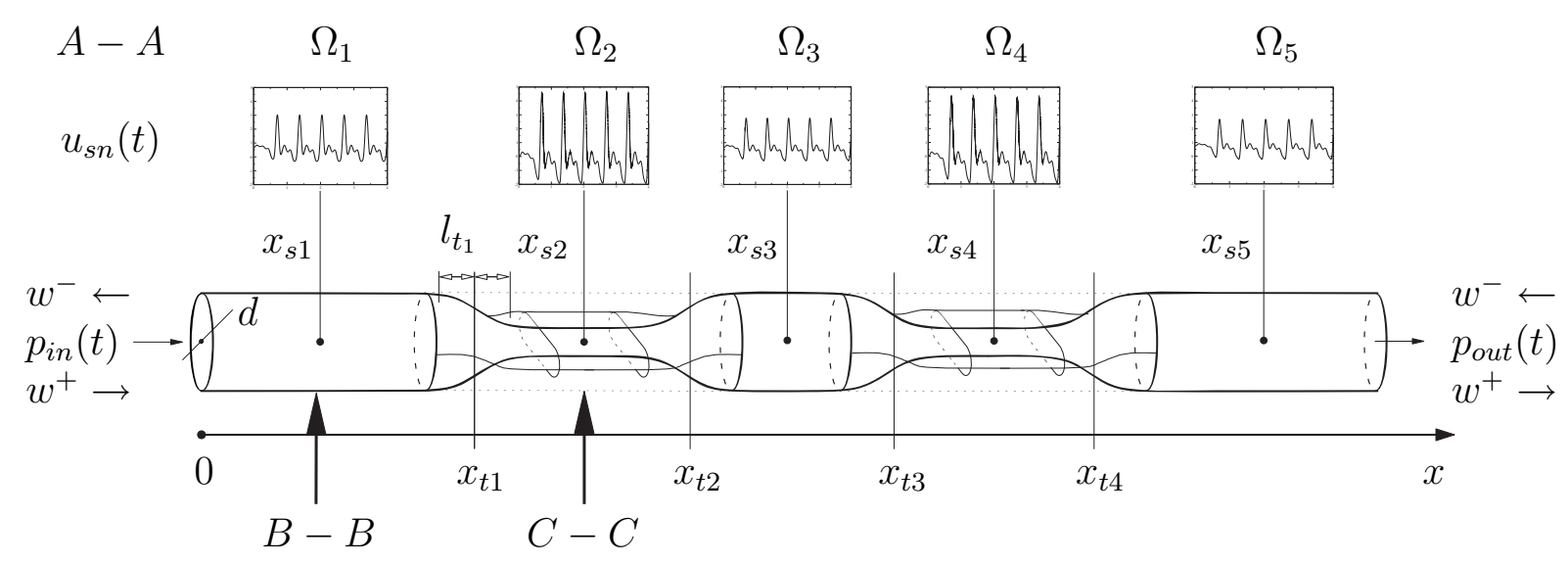

Figure 3.1: Schematic anatomy of a double myocardial bridge. The control segments $\Omega_{n}$ are equally spaced. Observation locations for haemodynamic properties are in the centre of each segment at $x_{s n}$, transitions between the segments are at $x_{t n}$. The transition length between the segments is denoted by $t_{l n}$. The graphs above each segment illustrate the differences in flow velocity $u_{s n}$. To illustrate the deformation we have indicated two cross-sections $B-B$ and $C-C$ in the circular and non-circular segments respectively (see figure 3.2). The pressure at the inlet and outlet are given by $p_{\text {in }}$ and $p_{\text {out }}$ respectively, the characteristics $\omega^{+}$and $\omega^{-}$are discussed in chapter 4 .

As shown in the angiography 2.5 and figure 3.1 the coronary arteries in myocardial bridges are structured by several wall deformations. Their number, degree and extension may independently vary with time, so that the axial curvature of the arterial wall for each of the $n=1 \ldots N$ myocardial bridges in series is characterised by $N$ functions. The deformation is specified by a parameter $\zeta$, defined as $\zeta=R_{d} / R_{0}$, which is chosen in the stenosis $n$ to vary with time as

$$
\zeta_{n}(t)=\frac{1}{2}\left(\zeta_{\text {diastole }}-\zeta_{\text {systole }}\right)\left(g_{n}(t)+1\right)+\zeta_{\text {systole }},
$$

where $g_{n}(t)$ are periodic functions in $t$ depending on the contraction of the muscle fibres. $\zeta_{\text {systole }}$ and $\zeta_{\text {diastole }}$ are fixed geometrical parameters between 0 and 1, specifying the degree of systolic and diastolic deformation respectively. We note that in the centre of the deformation $\zeta\left(x=x_{s 2}\right)=\zeta_{0}$, i.e. the degree of deformation increases with decreasing $\zeta_{0}$ and consequently $\zeta_{\text {systole }}<\zeta_{\text {diastole. }}$. To represent the time dependence of the deformation found in intra-vascular ultra-sound (IVUS) measurements [120], a synthetic deformation waveform $g_{n}(t)$ given by $\mathrm{m}=1 . .3$ sine/cosine harmonics is used.

$$
g_{n}(t)=\sum_{m=1}^{3} \frac{0.8}{m} \sin \left(m \omega\left(t-\triangle t_{n}\right)+\phi_{m}\right)
$$

Here $\Delta t_{n}$ is the delay for each deformation with respect to the cardiac cycle (see figure 3.3) and $\phi_{m}$ are the phases in radian, chosen to be $\phi_{1}=3.5, \phi_{2}=1.5$, and 


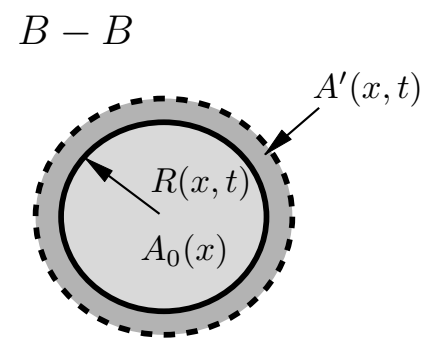

$$
C-C
$$

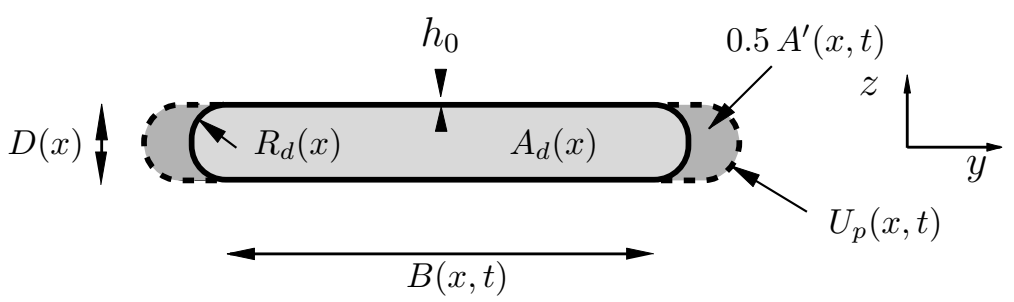

Figure 3.2: The deformation geometry of a linear elastic circular tube under negligence of bending stress inside the wall. Cross-section $B-B$ (left) shows circular expansion under pressure and the cross-section $C-C$ illustrates the geometry under external deformation independent of $z$ (right). The equilibrium cross-sectional area $A_{d}$ is shaded in light grey, while the perturbation area $A^{\prime}$ is shaded in dark grey. The dashed lines indicate expansion under pressure.

$\phi_{3}=3.9$. The axial curvature of each deformation is approximated by two hyperbolic tangent functions, so that

$$
R_{d}(x, t)=R_{0} \sum_{n=1}^{N} \zeta_{n}\left[\tanh \left(\frac{x-x_{t_{n}}}{l_{t_{n}}}\right)-\tanh \left(\frac{x-x_{t_{n+1}}}{l_{t_{n+1}}}\right)\right]
$$

where $x$ is the axial coordinate and $x_{t n}$ are the transition locations. Equation (3.30) smoothes the transition between the segmental domains $\Omega_{n}$ by a transition length $l_{t_{n}}$. The actual state of deformation can also be expressed by the ratio, $\varepsilon$, based upon the half-axes of the non-circular duct.

$$
\varepsilon(x, t)=\frac{R_{d}}{\frac{1}{2} B+R_{d}}
$$

\subsubsection{The pressure-area relationship for non-circular cross- sections}

DuE to its complicated structure, it is difficult to provide a synthetic mathematical description for the mechanical behaviour of vessel walls. Here, we focus on the most relevant structural features and the simplest mathematical model for arterial tissues and we subsequently derive an algebraic pressure-area relationship for a vessel under external deformation. In this context we characterise the relative change in crosssectional area with respect to the pressure increment according to $A=A\left(R_{d}, p\right)$.

INITIALLY we assume that the actual behaviour of the arterial tissue for small deformations does not depend on the strain rate, and that the deformation is symmetric 


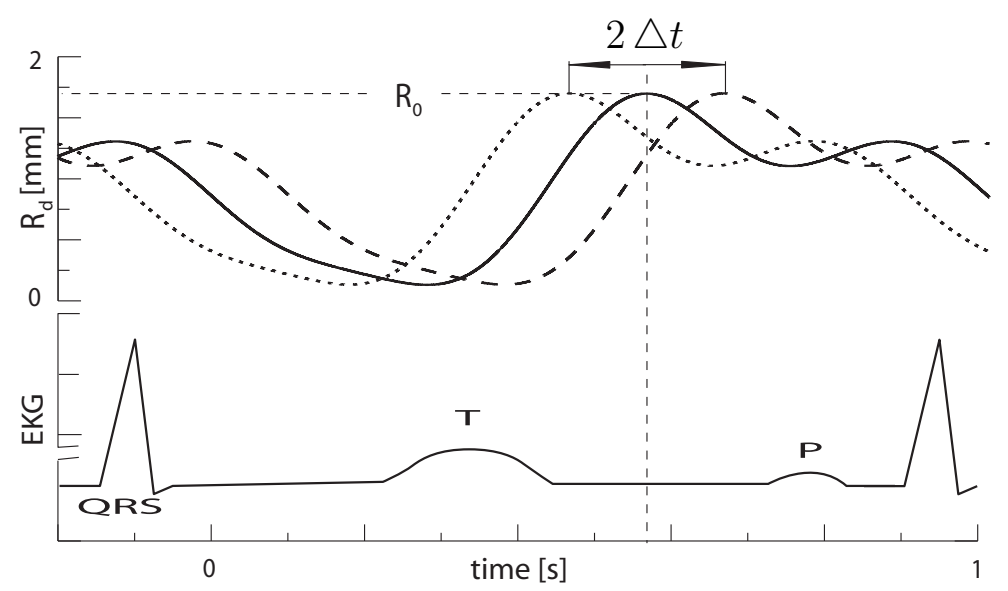

Figure 3.3: The variation of the bending radius $R_{d}\left(x_{s 2}, t\right)$ in the centre of the deformation is plotted with respect to the cardiac cycle, represented by an EKG trace. The deformation function (solid line) is shifted with respect to the cardiac cycle by $\Delta t=0 \pm 100 \mathrm{~ms}$ (dashed lines) to illustrate differences caused by the transit time of a pressure wave.

about the $x y$-plane. Under prior assumptions that the arterial wall is homogeneous and isotropic the theory of linear elastic shells is applicable [121]. The equation for $R^{\prime}$, the radial wall displacement of a infinite cylidrical shell is

$$
\frac{\partial^{2}}{\partial x^{2}}\left(\Theta \frac{\partial^{2} R^{\prime}}{\partial x^{2}}\right)+\frac{E h_{0}}{R^{2}} R^{\prime}=\Phi
$$

where $\Phi$ is the total force per unit area acting in the radial direction including the stresses induced by the fluid. Henceforth we assume that $\Phi$ is equal to the transmural pressure $p_{t m}$. The flexural rigidity $\Theta$ in equation (3.32) is given by

$$
\Theta=\frac{E h_{0}^{3}}{12\left(1-\sigma^{2}\right)}
$$

where $\sigma$ is Poisson's ratio. The assumption of material homogeneity and isotropy indicates that $E=E_{\theta}=E_{x}$ and $\sigma=\sigma_{\theta}=\sigma_{x}$, whereas typical values for biological tissues are $0.6 \mathrm{MPa}$ and 0.5 respectively.

THE first term in the shell equation describes the effect of transverse shear, the second one the membrane force along the tangent to the cross-section. It was experimentally shown that the longitudinal motion of the arterial wall is very small $[11,15]$. This behaviour is partially due to strong vascular tethering and partially due to the circumferential orientation of smooth muscles, through which the coupling of circumferential and longitudinal strain is minimised. Further we note that a thin shell has 
little resistance to bending and the shear forces are small compared with the extensional ones, so that we can provide an explicit relation between the pressure and the vessel cross-sectional area $A=\pi\left(R+R^{\prime}\right)^{2}$. We denote the perturbation about the equilibrium area $A_{d}\left(R_{d}\right)$ by $A^{\prime}\left(R_{d}, p\right)$, and hence the relation for the total cross-sectional area becomes dependent on $R_{d}$ and we may write $A\left(R_{d}, p\right)=A^{\prime}\left(R_{d}, p\right)+A_{d}\left(R_{d}\right)$. Under the above assumptions the shell-equation (3.32) is

$$
p=\epsilon E \frac{h_{0}}{R_{d}}=\varsigma_{h} \frac{h_{0}}{R_{d}},
$$

where $\varsigma_{h}$ is the hoop stress and $\epsilon$ is the circumferential strain given by

$$
\epsilon=\frac{U-U_{0}}{U_{0}}=\frac{2 \pi R_{d}+2 B-2 \pi R_{0}}{2 \pi R_{0}}=\frac{R_{d}}{R_{0}}+\frac{B}{\pi R_{0}}-1 .
$$

However if the tube is tethered against longitudinal displacements $\xi$, which is generally the case in the vasculature, a cancelling strain equal and opposite to

$$
\frac{\partial \xi}{\partial x}=-\frac{\sigma p R}{E h_{0}}
$$

must be supplied by applying longitudinal forces to the tube wall. The resultant longitudinal stress is comparable with the circumferential stress and produces just as that does, strains in perpendicular directions. The stress in a tethered tube is therefore given by

$$
\varsigma_{t}=p \frac{R_{d}}{h_{0}}=\epsilon \frac{E}{1-\sigma^{2}},
$$

Equation (3.35) and (3.37) lead to an expression for the pressure dependence of the width

$$
B\left(R_{d}, p\right)=\pi R_{0}\left[\frac{1-\sigma^{2}}{E} \frac{R_{d}}{h_{0}} p+1-\frac{R_{d}}{R_{0}}\right] .
$$

The total cross-sectional area is

$$
A\left(R_{d}, p\right)=\pi R_{d}^{2}+2 B\left(R_{d}, p\right) R_{d} .
$$

In the unperturbed state, the cross-section is

$$
A_{d}\left(R_{d}\right)=R_{d} U_{0}-\pi R_{d}^{2}
$$

We can finally write the pressure induced perturbation as

$$
A^{\prime}\left(R_{d}, p\right)=R_{d}^{2} U_{0} p \frac{1-\sigma^{2}}{E h_{0}} .
$$

Application of equation (3.34) and (3.39) leads to the pressure-area relation for $p \geq 0$

$$
p\left(R_{d}, A\right)=\frac{E h_{0}}{\left(1-\sigma^{2}\right)} \frac{\left(\pi R_{d}^{2}+A-R_{d} U_{0}\right)}{U_{0} R_{d}^{2}} .
$$


IT should be noted that under the assumption of linear elastic material with constant elastic modulus, equation (3.39) and (3.41) have the property that the area increases linearly with excess pressure. Real arteries, however, resist over-expansion by having an incremental elastic modulus $E(\epsilon)$ that increases with increasing strain [16]. It should be further noted that the area perturbation in equation (3.41) is not only dependent on pressure variation but also on the degree of deformation through $R_{d}$. This is due to the fact that the distensibility of the tube depends in some way on the wall geometry and its elastic properties. For a given cross-sectional area $A$ it is defined as

$$
\mathcal{D}_{A}:=\frac{1}{A}\left[\frac{\partial A}{\partial p}\right]_{A},
$$

which according to equation (3.39) is

$$
\mathcal{D}_{A}=\frac{2 R_{d}}{A} \frac{\partial B(\zeta, p)}{\partial p}=\frac{U_{0} R_{d}^{2}}{A} \frac{\left(1-\sigma^{2}\right)}{E h_{0}} .
$$

The distensibility $\mathcal{D}_{A}$ is directly related to the wave speed by

$$
c_{A}(x, t)=\frac{1}{\sqrt{\rho_{0}\left(\frac{1}{\kappa}+\mathcal{D}_{A}\right)}},
$$

As previously discussed $\kappa=0$ for incompressible fluids. The $\zeta_{0}$-dependence of the above relations is shown in figure 3.4.

AT negative transmural pressure, however, the original shape buckles due to bending of the vessel wall and eventually collapses. To account for the strongly nonlinear behaviour another vessel wall parameter can be introduced to determine the linear/non-linear nature of elastic response

$$
\epsilon=\left(\frac{U-U_{0}}{U_{0}}\right)^{\varpi / 2}
$$

Linear behaviour is obtained for $\varpi=2$, while for $\varpi>2$ or $\varpi<2$ the response is non-linear, i.e. stiffening or flagging of the wall. Consequently the width becomes

$$
B\left(R_{d}, p\right)=\pi R_{0}\left[\left(\frac{1-\sigma^{2}}{E} \frac{R_{d}}{h_{0}} p\right)^{2 / \varpi}+1-\frac{R_{d}}{R_{0}}\right]
$$

and the pressure area relation is

$$
p\left(R_{d}, A\right)=\frac{E}{1-\sigma^{2}} \frac{h_{0}}{R_{d}}\left[\frac{\left(\pi\left(R_{d}\right)^{2}+A-R_{d} U_{0}\right)}{R_{d} U_{0}}\right]^{\varpi / 2} .
$$

Finally the distensibility is

$$
\mathcal{D}_{A}=\frac{4 R_{d} A_{0}}{p \varpi A R_{0}}\left(\frac{1-\sigma^{2}}{E} \frac{R_{d}}{h_{0}} p\right)^{2 / \varpi} .
$$


(a) Compliance, area, wave and flow velocity versus deformation

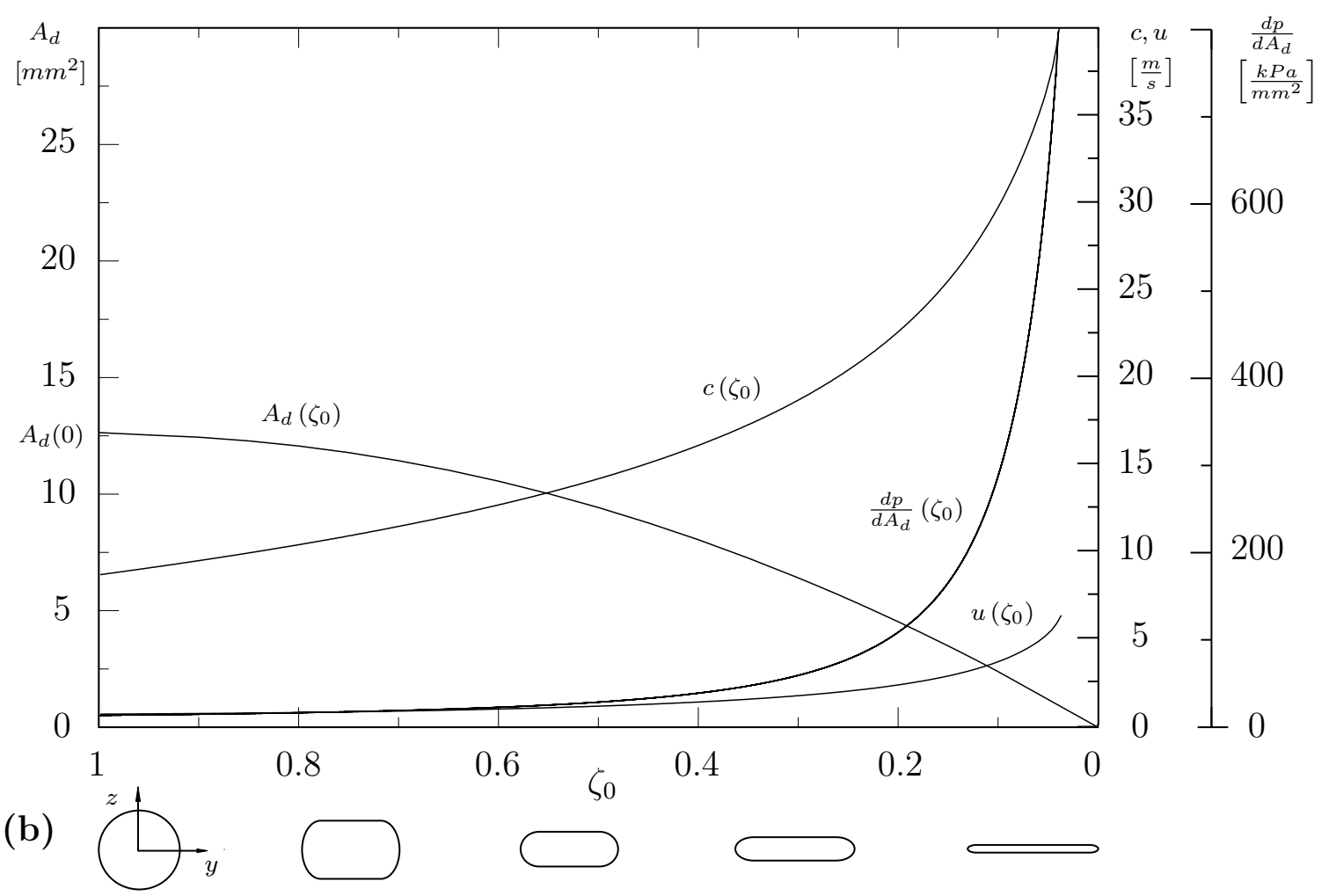

Figure 3.4: (a) Dependence of compliance, area, wave and flow velocity on deformation $\zeta_{0}$. The gradient $d p / d A_{d}=\frac{1}{\mathcal{D}_{A_{d}} A_{d}}$ is the compliance per unit length. (b) Schematic illustration of the different stages of deformation.

\subsubsection{Elastic modulus and wall thickness as a function of tube radius}

As the model depends on the elasticity of the wall, their thickness and the vessel radius, we need to find a functional relation to measurements of the volume compliance given in [122]. The volume compliance is approximately given by

$$
C_{v o l}=\frac{d \mathscr{V}}{d p} \approx \frac{3 A_{0} L R_{0}}{2 E h_{0}}
$$

where $\mathscr{V}$ is the volume of a given segment and $A_{0}$ is the average cross-sectional area. Following suggestions in [18] a relation for elastic properties is obtained by an 
exponential curve fit to above data so that

$$
\frac{E h_{0}}{R_{0}}=k_{1} \exp \left(k_{2} R_{0}\right)+k_{3},
$$

with the constants $k_{1}, k_{2}$, and $k_{3}$. With data for $C_{v o l}$ from Westerhof [26], Stergiopulos [122], and Segers [123] (shown in figure 3.5) we obtain $k_{1}=2.0 * 10^{6} \mathrm{~N} \mathrm{~m}^{-2}$, $k_{2}=-2.253 * 10^{3} \mathrm{~m}^{-1}$, and $k_{3}=8.65 * 10^{4} \mathrm{~N} \mathrm{~m}^{-2}$.

Elastic wall properties in dependence on vessel radius

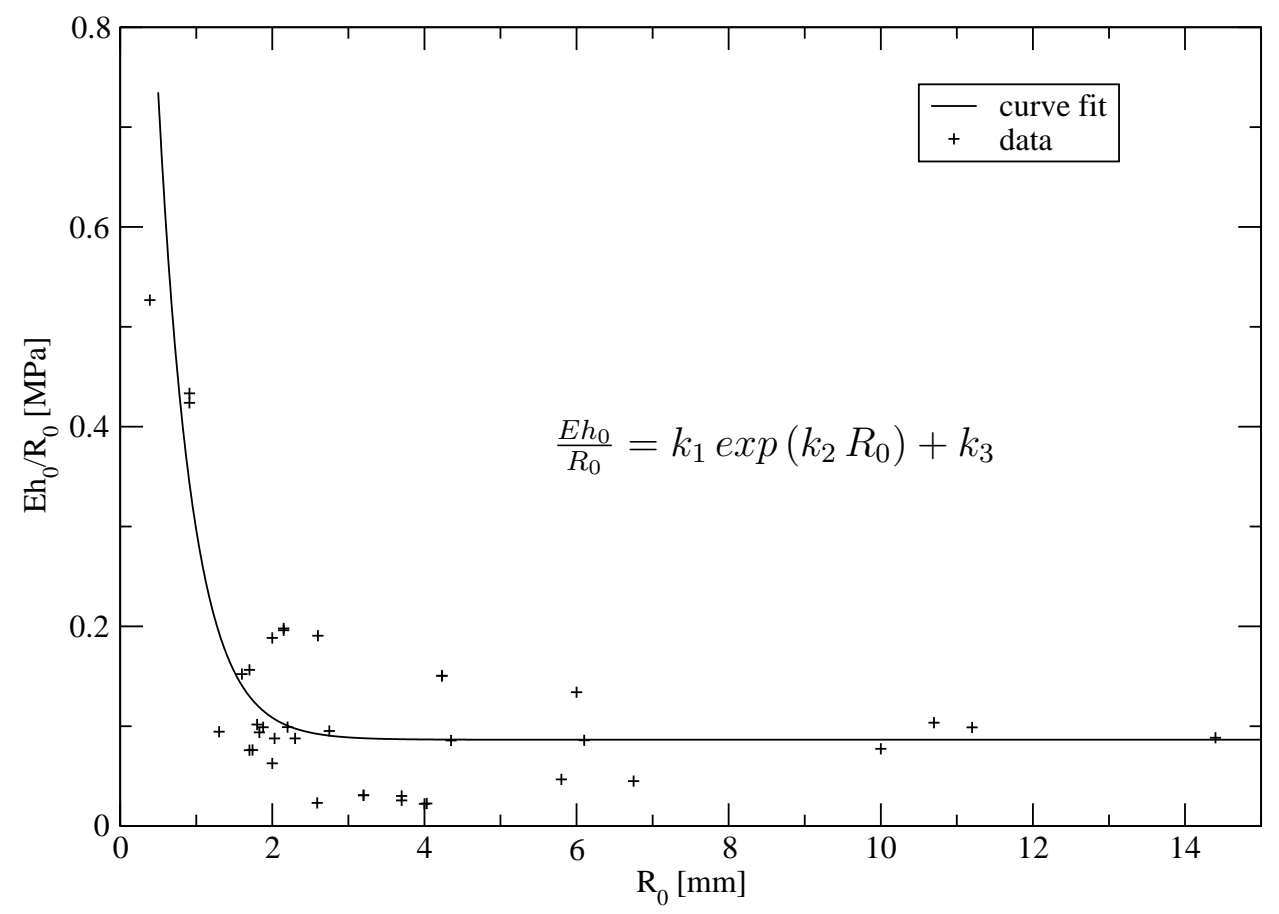

Figure 3.5: Curve fit (solid line) with a function of the form (3.51) to data [122] (crosses) converted by equation (3.50), both plotted over the tube radius $R_{0}$.

A functional relationship for the wall thickness of small to medium sized arteries subject to the vessel radius can be found in [124], where

$$
h_{0}=a R_{0}^{b} .
$$

The parameters for $a=3.87$ and $b=0.63$ were obtained by a logarithmic fit to data including vessel radii between $100 \mu \mathrm{m}$ and $3000 \mu \mathrm{m}$. For larger arteries the ratio of wall thickness and radius remained remarkably constant so that $h_{0} / R_{0} \approx 0.16$ [125]. 
Both relations are shown in figure 3.6. We note that the wall thickness, the diameter and the elastic modulus of the vessel are difficult to measure because they change with pressure and age and show significant variation in different persons. For example the elastic modulus of the thoracic aorta varies between $0.036 \mathrm{MPa}$ and $0.409 \mathrm{MPa}$; the lowest values were found in young people ( $<30$ years). In general many of the parameters vary significantly. Here we are interested in modelling a representative physiological behaviour and not one specific case. However, it was found that the thickness to diameter ratio is approximately constant for all ages and that the elastic properties are well described by equation (3.51) [20].

Wall thickness in dependence on vessel radius

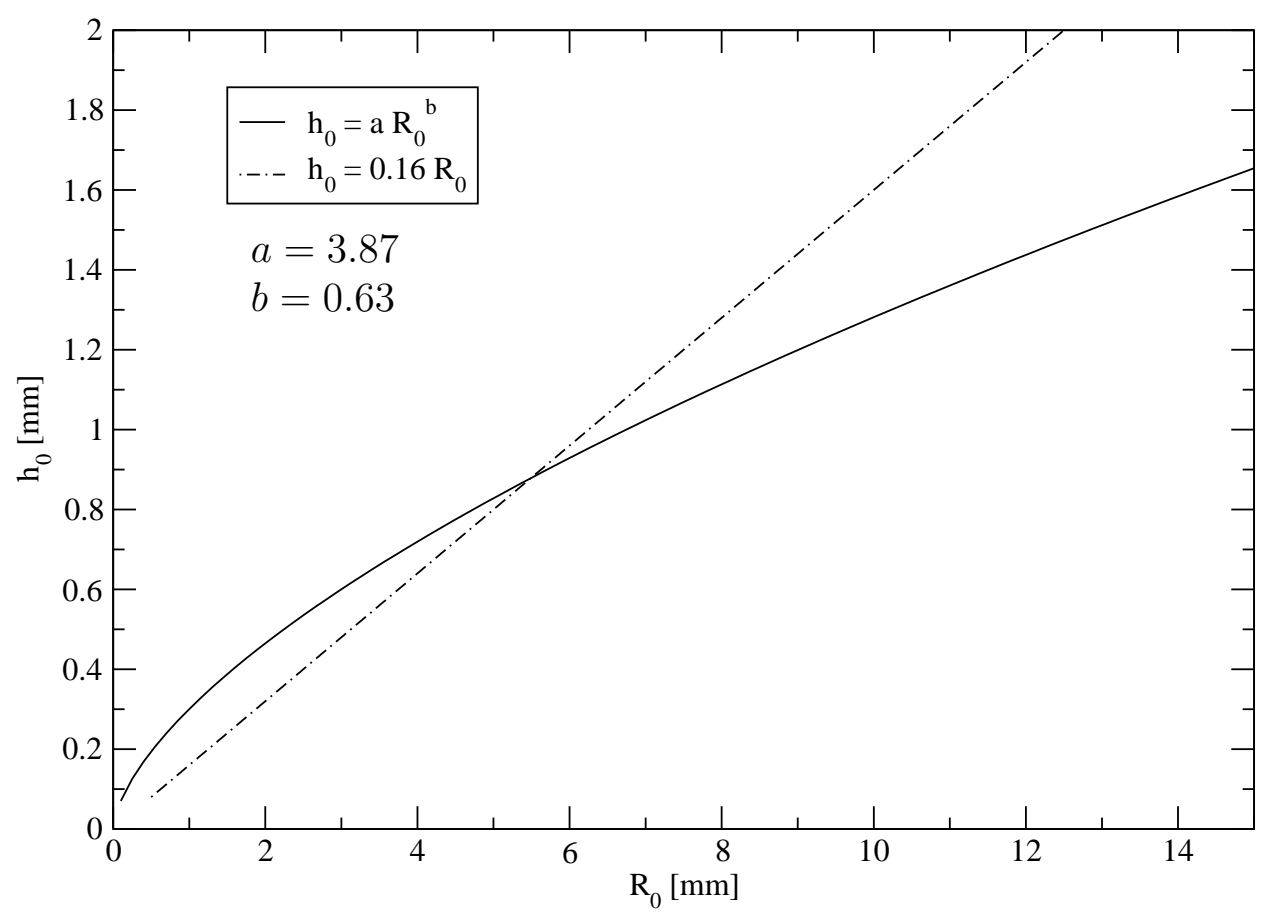

Figure 3.6: Curve fit with a function of the form (3.52) (solid line) to data in [124] compared to the relation for larger arteries with approximately constant wall thickness to diameter ratio (dashed line).

WE use equation (3.51) and (3.52) to determine the wall thickness and elastic properties from the vessel radius for the following reasons: Firstly we are mainly concerned with the circulation in the coronary arteries and secondly that no indications were found that the functional dependence in equation (3.52) cannot be continued to 
larger radii. Consequently the relation for the elastic modulus is choosen as

$$
E\left(R_{0}\right)=\frac{R_{0}^{(1-b)}}{a}\left(k_{1} \exp \left(k_{2} R_{0}\right)+k_{3}\right)
$$

In figure 3.7 we compare elastic moduli for both relations as a function of vessel radius. It should be noted that the values of elastic modulus and wall thickness are not required separately and that the relation in equation (3.51) is significant.

Elastic modulus in dependence on the vessel radius

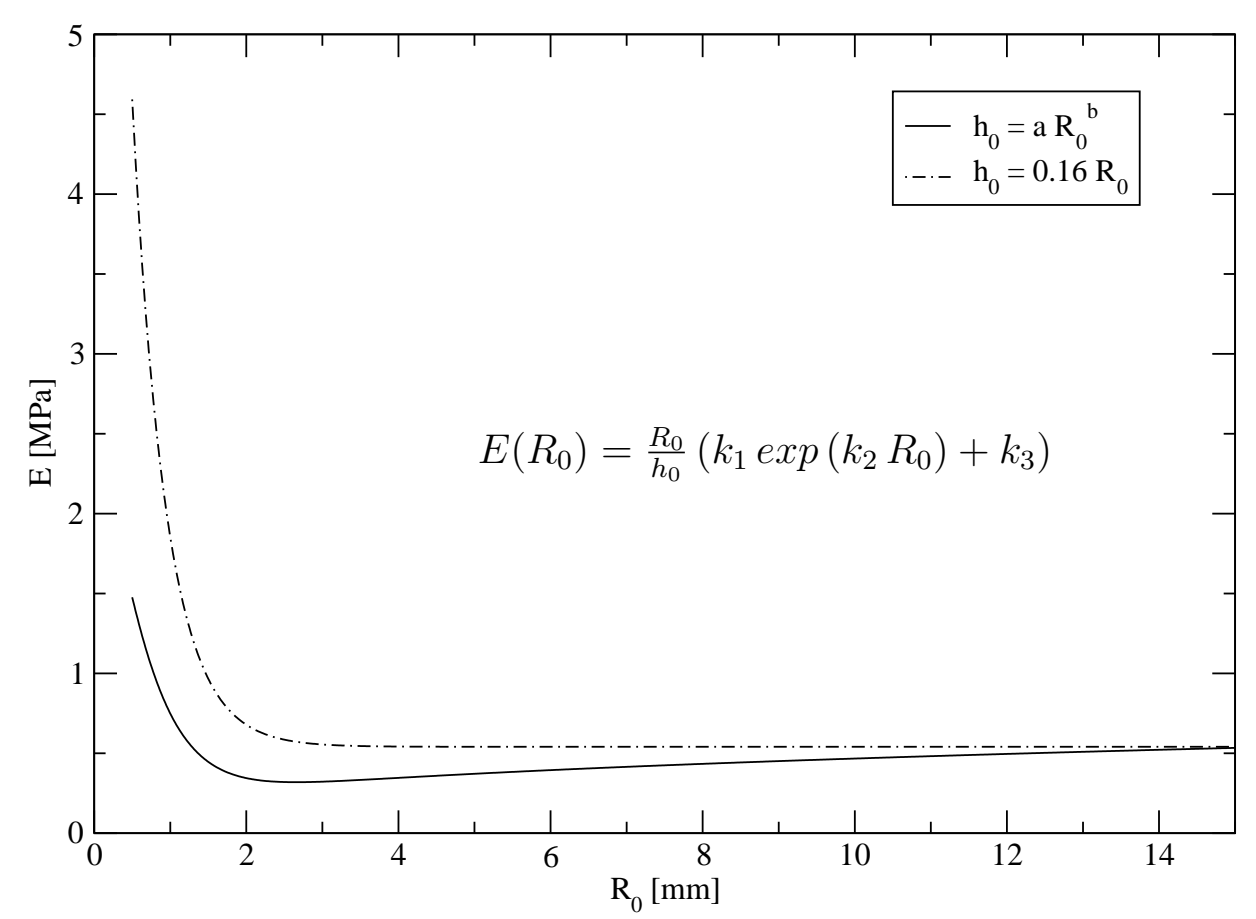

Figure 3.7: Elastic modulus obtained by relation (3.51) (solid line) and by linear relation (dashed line) between the radius and wall thickness of the vessel.

FinALLY we need to know if the assumptions made in the previous section are well satisfied for these relations. The ratio of wall thickness to radius for a typical vessel under consideration is below $h_{0} / R_{0}=0.21$, so that the condition $a R_{0}^{(b-1)} \ll 1$ is sufficient. 


\subsection{Influence of viscosity}

So far we have derived a model for pulsatile fluid flow in a time dependent quasione-dimensional vessel geometry, however we have not specified the variation of flow profiles and the influence of fluid viscosity on the flow. A detailed local description of these flow profiles is needed to characterise the viscous friction and the pressure drop. In this section we discuss three types of laminar flow - (i) fully developed Poiseuille flow, (ii) oscillating pipe flow and finally we present (iii) a new boundary layer method to simulate developing flow conditions in the vasculature.

\subsubsection{Laminar fully developed flow}

THE simplest model for blood flow through a vessel would be the simulation of steady, fully developed flow of a Newtonian fluid through a rigid cylindrical tube of circular cross-section. Such flows are characterised as Poiseuille flow in the honour of J. L. M. Poiseuille who performed experiments relating pressure gradient, flow and tube geometry. A quasi-stationary approximation to pulsatile flow is acceptable for small Womersley numbers, where the viscous term is well approximated by the steadyflow solution. Under these conditions the velocity profile is independent of the axial position and time. The viscous term $F_{\nu}$ in equation (3.24b) is therefore homogeneous, which leads to $\chi$ being a constant. A typical approximation for pulsatile laminar flow in small axially symmetric vessels is a flow profile of the form

$$
v_{x}=u \frac{\gamma+2}{\gamma}\left[1-\left(\frac{r}{R}\right)^{\gamma}\right]
$$

where $u$ is the mean velocity along the axis of the tube and $R$ is the radius of the tube, while $\gamma$ is the profile exponent. We note that $\gamma=2$ corresponds to the Newtonian fluid where the flow profile is parabolic (Hagen-Poiseuille flow, see [75]). However other values of $\gamma$ are used to fit experimental data, for example $\gamma=9$ is closer to the plug flow profile; it describes the flow of a non-Newtonian fluid reflecting the fact that blood is a suspension of cellular elements in plasma. We differentiate the viscous term on the right hand side of the momentum equation in (3.24b) to obtain

$$
F_{\nu}=2 \pi \nu R\left(-\frac{(\gamma+2) u}{R}\right)=-2 \pi \nu(\gamma+2) u=K_{\nu} u
$$

whereas the friction coefficient for a parabolic profile is $K_{\nu}=-8 \pi \nu$. The corresponding momentum correction coefficient per definition is

$$
\chi=\frac{\gamma+2}{\gamma+1}
$$


which is $\frac{4}{3}$ in the parabolic case. Rearrangement leads to the following relationship between the shape of the velocity profile determined by $\gamma$ and the correction coefficient

$$
\gamma=\frac{2-\chi}{\chi-1}
$$

so that for a non-uniform velocity profile $(\chi \neq 0)$ the friction term can be expressed as

$$
F_{\nu}=-2 \pi \nu u \frac{\chi}{\chi-1} .
$$

Such factors can be used for the purpose of correlating other variables as well as for direct calculation of pressure drop. We note that in the presence of a stenosis the total losses are underestimated [72, 122]. This is mainly due to underestimation of viscous forces and disregarding the losses caused by flow separation at the diverging end of the stenosis $[12,114]$.

\subsubsection{Oscillating pipe flow}

GENERALLY, the system is much more dynamic (high Womersley numbers) and the velocity profile changes with oscillatory frequency. Measurements of pulsatile velocity profiles in the vasculature reveal rather uniform core profile with a thin boundary layer region [126]. The thickness $\delta_{s t}$ of the Stokes layer in a large tube with an oscillating fluid core can be estimated according to Stokes [7] by the following relation:

$$
\delta_{s t} \approx \sqrt{\frac{\nu}{\omega}}
$$

where $\omega=2 \pi f$ and $f$ is the frequency of oscillation (heart beat), which is of order $\mathcal{O}(1) H z$. The kinematic viscosity of blood is of the order $\nu \approx \mathcal{O}\left(10^{-6}\right) \frac{m^{2}}{s}$, so that $\delta_{s t} \approx \mathcal{O}\left(10^{-4}\right) m$, which is much smaller than the radius of the vessel. The Stokes layer thickness is closely related to the Womersley number so that

$$
W_{O}=\sqrt{\frac{\omega A}{\nu}} \approx \frac{\sqrt{A}}{\delta_{s t}}
$$

The vessel radius is of the order $R \approx \mathcal{O}\left(10^{-3}\right) m$, thus the Womersley number takes values of $W_{o} \approx \mathcal{O}(10)$. Therefore the transient inertial forces are large compared to the viscous forces and the velocity profile is rather uniform. Following the derivation in [20] such a flow can be approximated by the following velocity profile:

$$
v_{x}=\left\{\begin{array}{cl}
u & r \leq R-\delta_{s t} \\
u(R-r) / \delta_{s t} & R-\delta_{s t}<r \leq R
\end{array}\right.
$$

In this case

$$
F_{\nu}=-\frac{2 \pi \nu u R}{\delta_{s t}}
$$


Boundary layer thickness in dependence on frequency

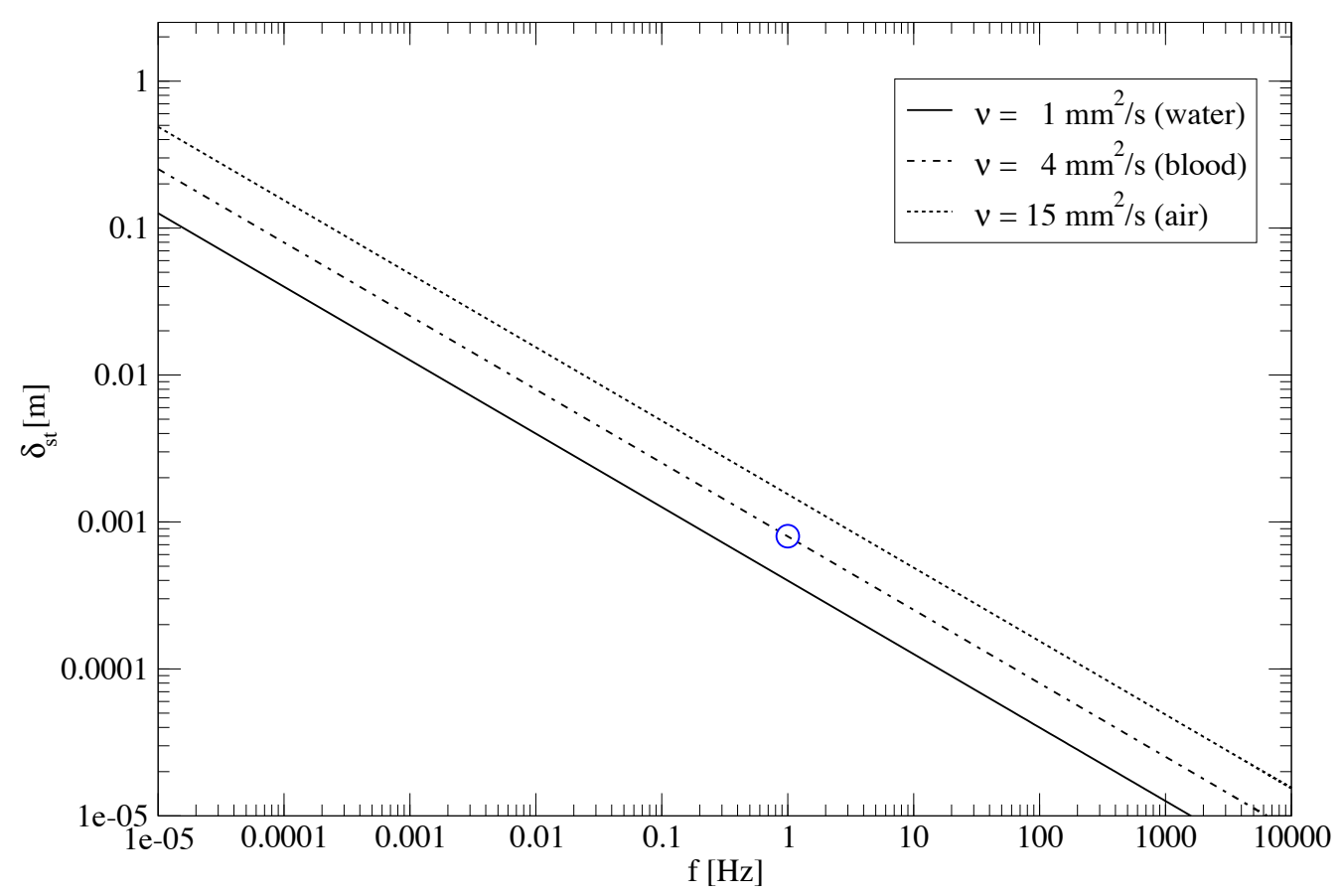

Figure 3.8: Stokes layer thickness (3.59) for water, blood and air plotted against the frequency in Hertz.

and

$$
\chi=\frac{1}{A u^{2}}\left[\int_{0}^{R-\delta_{s t}} 2 \pi r u^{2} d r+\int_{R-\delta_{s t}}^{R} 2 \pi r\left(\frac{u(R-r)}{\delta_{s t}}\right)^{2} d r\right]=1-\frac{4 \delta_{s t}}{3 R}+\frac{\delta_{s t}^{2}}{2 R^{2}} \approx 1 .
$$

\subsubsection{Developing flow}

We remark that in blood flow applications the velocity profile depends on both $x$ and $t$, so that the current one-dimensional models can be generalised. Analysis which studies the influence of the spatial and temporal variations in the velocity profile have not been performed and are an interesting topic for further research. In the following section we investigate the solutions of the unsteady boundary layer equations by using an approximate integral method proposed by Veldman[83, 84]. For this purpose, the potential flow of the two-dimensional equations governing the unsteady incompressible laminar boundary layer flow under axial symmetry is taken to be in power-law form. 
By the introduction of similarity variables and the assumption that the evolution of the velocity profile is weakly dependent on axial coordinate $x$, the boundary layer equations reduce to the Falkner-Skan equation. Based on this ordinary differential equation, closed form solutions to the von Kármán integral momentum equation are obtained by a curve fit representation given in [80]. The skin friction and momentum correction coefficients corresponding to the velocity distributions are compared with known results in steady flow conditions. In particular the results are found to compare favourably with the Blasius solution and values for fully developed flow.

\section{Entrance region}

BLOOD ejected from the heart flows from a pressure reservoir into successively smaller arteries. Sometimes the flow is not fully developed in the entrance region of arteries descending from large vessels like the aorta. Flow in these regions is similar to an entrance flow with a potential core and a developing boundary layer at the wall. The velocity profiles are nearly uniform at the entrance, however frictional forces at the wall retard the flow and the thickness of the boundary layer grows, while (due to continuity requirements) the core velocity increases (see figure 3.9). Some distance

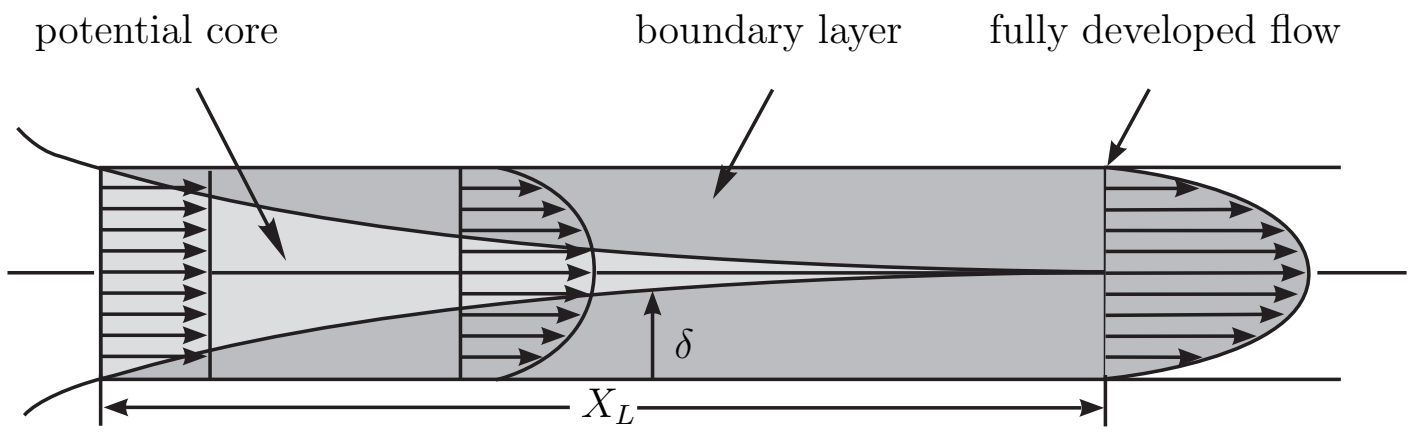

Figure 3.9: Steady developing flow in the entrance of a circular tube. The thickness $\delta$ of the boundary layer (dark grey) increases until the flow is fully developed. In the potential core (light grey) the fluid is assumed to be inviscid. The development of flow profile is schematically shown.

downstream the shear layers merge, and the tube is entirely filled with the boundary layer. To determine if the flow has reached its steady-state behaviour (i.e in which the profile does not change with $x$ ), the concept of entrance length was introduced. The entrance length, $X_{L}$, is sometimes defined as the point where the centreline velocity equals $99 \%$ of the maximum Poiseuille value [76], so that

$$
\frac{X_{L}}{d}=\frac{0.6}{1+0.035 R e_{d}}+0.056 R e_{d}
$$


where $d=2 R$ is the diameter of the tube. The flow is developing in regions $0 \leq x \leq$ $X_{L}$, while it is fully developed beyond $x=X_{L}$. In resting flow conditions the entrance length for a vessel under consideration $(R e=350, d=4 \mathrm{~mm})$ is $X_{L} \approx 78 \mathrm{~mm}$, however, in elevated flow conditions $\left(R e=1000, d=4 \mathrm{~mm}\right.$ ) it may reach $X_{L} \approx$ $225 \mathrm{~mm}$, so that the flow in the left main coronary artery is under no circumstances fully developed. Likewise the flow in the entrance region of these arteries is subject to viscous forces that cannot be neglected. Of particular interest is the excess pressure drop compared to Poiseuille flow, which is due to increased shear in the entrance boundary layers and the acceleration of the core flow.

THE pulsatile nature of blood flow in the entrance region is dependent on Womersley and Reynolds number. At low Womersley numbers, the unsteady and steady entrance length are approximately the same and mainly dependent on the Reynolds number. As discussed earlier the thickness of the boundary layer is more uniform during the cardiac cycle at high Womersley numbers.

\section{Boundary layer equations}

THE notion of the boundary-layer approximations was first developed by Ludwig Prandtl in the early 1900's. These well-known approximations are widely applied in fluid mechanics [75]. They apply to flows where there are extensive inviscid regions separated by thin shear layers of typical width $\delta \ll \ell$. As the flow rate in the vessel increases (i.e. at high Reynolds number) the boundary-layer approximations become increasingly valid. The derivation of the axial boundary layer equations was first given by Mangler (1945) and can be found in [76]. As previously assumed the circumferential velocity $v_{\theta}=0$ (no swirl in A), so that we start form the axially symmetric form of the continuity equation (3.6) and the momentum equations (3.7) and (3.10), both in dimensionless form. However, since in the limit $R e \rightarrow \infty$ these equations reduce to those for inviscid flow (non-slip), we apply the following boundary layer transformations

$$
\hat{r}=r^{*} \sqrt{R e} \sim \frac{r^{*}}{\delta^{*}}, \quad \hat{v}_{r}=v_{r}^{*} \sqrt{R e}
$$

which stretch $r^{*}$ and $v_{r}^{*}$ by a power of the Reynolds number, so that at least one friction term remains as $R e \rightarrow \infty$. The dimensionless form of the boundary layer equations is

$$
\begin{aligned}
\frac{\partial \hat{r} v_{x}^{*}}{\partial x^{*}}+\frac{\partial \hat{r} \hat{v}_{r}}{\partial \hat{r}} & =0 \\
\frac{\partial v_{x}^{*}}{\partial t^{*}}+v_{x}^{*} \frac{\partial v_{x}^{*}}{\partial x^{*}}+\hat{v}_{r} \frac{\partial v_{x}^{*}}{\partial \hat{r}} & =-\frac{\partial p^{*}}{\partial x^{*}}+\frac{\partial^{2} v_{x}^{*}}{\partial \hat{r}^{2}} \\
\frac{\partial p^{*}}{\partial \hat{r}} & =0 .
\end{aligned}
$$


On the basis of the above scaling arguments it then follows that

$$
\delta^{*} \sim \ell R e^{-\frac{1}{2}} \ll \ell
$$

i.e. the boundary layer tends to zero for large Reynolds numbers and is small compared to the body dimension. Further it can be deduced that the key approximations in classical boundary layer theory are that the pressure is constant across the shear layer and that stream-wise diffusion is negligible, i.e. for any variable $\square$

$$
\frac{\partial^{2} \bullet}{\partial \hat{r}^{2}} \gg \frac{\partial^{2} \bullet}{\partial x^{* 2}}
$$

Since the pressure does not vary across the boundary layer we can evaluate the gradient $\partial p^{*} / \partial x^{*}$ at the outer edge of the boundary layer, where the longitudinal velocity $v_{x}^{*}$ approaches asymptotically the free-stream velocity $V^{*}\left(x^{*}, t^{*}\right)$. Since the gradients $\partial v_{x}^{*} / \partial \hat{r}$ and $\partial^{2} v_{x}^{*} / \partial \hat{r}^{2}$ vanish there, the pressure is related to the potential flow by

$$
\frac{\partial V^{*}}{\partial t}+V^{*} \frac{\partial V^{*}}{\partial x^{*}}=-\frac{\partial p^{*}}{\partial x^{*}}
$$

This relation is used to eliminate equation (3.68), so that we obtain two equations for two unknowns $v_{x}^{*}\left(x^{*}, \hat{r}, t^{*}\right)$ and $\hat{v}_{r}\left(x^{*}, \hat{r}, t^{*}\right)$ of the boundary layer.

$$
\begin{aligned}
\frac{\partial \hat{r} v_{x}^{*}}{\partial x^{*}}+\frac{\partial \hat{r} \hat{v}_{r}}{\partial \hat{r}} & =0 \\
\frac{\partial v_{x}^{*}}{\partial t^{*}}+v_{x}^{*} \frac{\partial v_{x}^{*}}{\partial x^{*}}+\hat{v}_{r} \frac{\partial v_{x}^{*}}{\partial \hat{r}} & =\frac{\partial V^{*}}{\partial t^{*}}+V^{*} \frac{\partial V^{*}}{\partial x^{*}}+\frac{\partial^{2} v_{x}^{*}}{\partial \hat{r}^{2}} .
\end{aligned}
$$

The difference to plane flow emerges only in the continuity equation, so that a notation for both, plane boundary layers $(j=0)$ and axisymmetric boundary layers $(j=1)$ is frequently used. In dimensional form these equations are

$$
\begin{aligned}
\frac{\partial r_{w}^{j} v_{x}}{\partial x}+\frac{\partial r_{w}^{j} v_{r}}{\partial r} & =0 \\
\frac{\partial v_{x}}{\partial t}+v_{x} \frac{\partial v_{x}}{\partial x}+v_{r} \frac{\partial v_{x}}{\partial r} & =\frac{\partial V}{\partial t}+V \frac{\partial V}{\partial x}+\frac{1}{\rho_{0}} \frac{\partial \tau}{\partial r}
\end{aligned}
$$

where $r_{w}(x, t)$ is the local surface radius of the wall measured from the axis and $\tau$ represents the shear stress, which is defined as $\tau:=\mu \frac{\partial v_{x}}{\partial r}$. The derivation assumes that $r_{w}$ is much larger than the boundary layer thickness $\delta$. We note that the boundary layer equations are still valid if the boundary layers exist on the inner side of the body (as is the case in a vessel), which can be seen by changing the signs of $r_{w}$ and $v_{r}$ simultaneously. Consequently the appropriate boundary conditions for flow in a tube with moving walls are:

$$
\begin{array}{ll}
r=0 \quad: & v_{x}(x, 0, t)=V(x, t) \quad v_{r}(x, 0, t)=0, \\
r=R_{d}: & v_{x}\left(x, R_{d}, t\right)=0 \quad v_{r}\left(x, R_{d}, t\right)=-v_{w}(x, t),
\end{array}
$$


where $v_{w}$ is the normal velocity of the wall, which is positive during outward motion. The minus sign regards the fact that the wall normal to the vessel wall and the normal of the boundary layer have opposite signs.

THE aim is to solve these equations for a given core flow distribution $V(x, t)$. One way is to use approximate integral methods, which describe the evolution of the boundary layer including the thickness and viscous forces. However, the threedimensionality of deformation generally makes it difficult to find a satisfactory solution for every compartment of the neither circular nor flat duct. If we consider severe deformations (e.g. $\zeta_{0}=0.2$ ) the circumferential length of the flat portion of the vessel exceeds the circumferential length of the circular portion by a factor of four $\left(\frac{B}{\pi R_{d}}=\frac{1}{\zeta_{0}}-1\right)$, thus we assume plane wedge flow for the calculation of viscous forces (i.e. $j=0$ ). Consequently $r_{w}$ is the body shape along the $x r$-section of the tube, which is equal to $R_{d}$, and $v_{w}$ is taken to be the velocity component normal to the flat portion of the wall, which is

$$
v_{w}=\frac{\partial R_{d}}{\partial t}
$$

\section{Integral boundary layer equations}

THE momentum integral relation of von Kármán (1921) is obtained by multiplying the continuity equation (3.74) by $v_{x}-V$ and subtracting it from the momentum equation (3.75).

$$
-\frac{1}{\rho_{0}} \frac{\partial \tau}{\partial r}=\frac{\partial\left(V-v_{x}\right)}{\partial t}+\left(V-v_{x}\right) \frac{\partial V}{\partial x}+\frac{\partial\left(r_{w}^{j} v_{x} V-r_{w}^{j} v_{x}^{2}\right)}{\partial x}+\frac{\partial\left(r_{w}^{j} v_{r} V-r_{w}^{j} v_{r} v_{x}\right)}{\partial r} .
$$

Integration over the thickness of the boundary layer, i.e. from the wall at $r=R_{d}$ to the axis of the tube at $r=0$, which by the definition $\delta \ll R_{d}$ is always outside the boundary layer, leads to

$$
\begin{array}{r}
-\frac{\partial}{\partial r} \int_{R_{d}}^{0} \frac{\tau}{\rho_{0}} d r=\frac{\partial}{\partial t} \int_{R_{d}}^{0}\left(V-v_{x}\right) d r+\frac{\partial V}{\partial x} \int_{R_{d}}^{0}\left(V-v_{x}\right) d r \\
+\frac{\partial}{\partial x} \int_{R_{d}}^{0} r_{w}^{j} v_{x}\left(V-v_{x}\right) d r+\frac{\partial}{\partial r} \int_{R_{d}}^{0} r_{w}^{j} v_{r}\left(V-v_{x}\right) d r .
\end{array}
$$

Definition of the integral relations for the displacement thickness

$$
\delta^{*}(x, t):=\int_{R_{d}}^{0}\left(1-\frac{v_{x}}{V}\right) d r,
$$

the momentum thickness

$$
\theta(x, t):=\int_{R_{d}}^{0} \frac{v_{x}}{V}\left(1-\frac{v_{x}}{V}\right) d r,
$$


and the total displacement thickness $\delta^{* *}=\delta^{*}+\theta$

$$
\delta^{* *}(x, t):=\int_{R_{d}}^{0}\left(1-\frac{v_{x}^{2}}{V^{2}}\right) d r,
$$

and regarding the fact that centreline values of both $\tau$ and $v_{r}$ vanish and that $v_{x}$ is zero at the wall we obtain the compact form

$$
\begin{array}{r}
\frac{1}{V^{2}} \frac{\partial V \delta^{*}}{\partial t}+\frac{\partial \theta}{\partial x}+\frac{2 \theta+\delta^{*}}{V} \frac{\partial V}{\partial x}+\frac{\theta}{r_{w}^{j}} \frac{\partial r_{w}^{j}}{\partial x}-\frac{v_{w}}{V}-\frac{c_{f}}{2}=0 \\
\text { with } \quad u(0, t)=V(0, t), \quad \theta(0, t)=\delta^{*}(0, t)=0 .
\end{array}
$$

Here $c_{f}$ is a non-dimensional friction factor defined as

$$
c_{f}(x, t):=\frac{\tau_{w}}{\frac{1}{2} \rho_{0} V^{2}},
$$

where $\tau_{w}=\mu\left(\partial v_{x} / \partial r\right)_{w}$ is the wall shear stress. The only difference to plane flow is the term involving $\partial r_{w}^{j} / \partial x$. If $r_{w}^{j} \rightarrow \infty$ or $\partial r_{w}^{j} / \partial x \rightarrow 0$, equation (3.85) reduces to the von Kármán integral momentum equation for plane flow $(j=0)$. Compared to the frictional term $c_{f} / 2$ the influence of the term involving $\partial r_{w}^{j} / \partial x$ on the boundary layer properties is indeed small (below 0.3\%), thus disregarding the term by setting $j=0$ is appropriate. The boundary conditions in (3.86) assume a uniform inflow profile with velocity $V$.

\section{Falkner-Skan equation}

SuitABLE solutions to the boundary layer equations in either plane or axial symmetry are found by the introduction of the stream function $\psi$. For plane flow the relations are

$$
v_{x}=\frac{\partial \psi}{\partial y}, \quad v_{y}=-\frac{\partial \psi}{\partial x} .
$$

Thus by substitution the momentum equation (3.75) for plane flow $(r \rightarrow y)$ takes the following form

$$
\frac{\partial^{2} \psi}{\partial t \partial y}+\frac{\partial \psi}{\partial y} \frac{\partial^{2} \psi}{\partial x \partial y}-\frac{\partial \psi}{\partial x} \frac{\partial^{2} \psi}{\partial y^{2}}=\frac{\partial V}{\partial t}+V \frac{\partial V}{\partial x}+\frac{1}{\rho_{0}} \frac{\partial^{3} \psi}{\partial y^{3}}
$$

A coordinate transformation $x \rightarrow \xi$ and $y \rightarrow \eta$ turns the equation for the stream function into the Görtler equation [75]. The following approach mainly consists in assuming that the flow is locally self-similar and that it depends weekly on the coordinate $\xi$, so that the velocity profiles can be mapped onto each other by suitable scaling factors in $y$. Falkner and Skan have found a family of similarity solutions, where the free-stream velocity is of the power-law form

$$
V(x)=C x^{n},
$$


with a constant $C$ and the power-law parameter $n$. The similarity variable $\eta \sim y / \delta(x)$ is set as

$$
u(x, y)=V(x) f^{\prime}(\eta) \text { where } \eta=y \sqrt{\frac{(1+n) V(x)}{2 \nu x}},
$$

where $f(\eta)$ is the (self-similar) stream function and the prime refers to derivative with respect to $\eta$. The coordinate normal to the plate is denoted by $y$. However, there are other more general similarity solutions including the temporal dependence of the profile evolution [127]. The above similarity variables turn the boundary layer equations into a non-linear ordinary differential equation of order three, which is known as the Falkner-Skan-Equation

$$
\begin{array}{rlll}
f^{\prime \prime \prime}+f f^{\prime \prime}+\beta\left(1-f^{\prime 2}\right) & =0 & \text { where } & \beta=\frac{2 n}{1+n} \\
\text { with } \quad f(0)=f^{\prime}(0) & =0 & \text { and } \quad \lim _{\eta \rightarrow \infty} f^{\prime}(\eta)=1 .
\end{array}
$$

The parameter $\beta$ is a measure of the pressure gradient $\partial p / \partial x$. If $\beta$ is positive, the pressure gradient is negative or favourable, $\beta=0$ indicates no pressure gradient (i.e. the Blasius solution to flat plate flow) and negative $\beta$ denotes a positive or unfavourable pressure gradient. We note that by assumption, $\beta$ should vary slowly with coordinate $x$. The solutions are found numerically by a shooting method with $f^{\prime \prime}(0)$ as free parameter [128]. To avoid extensive calculations we follow a curve fit representation of three quantities extracted from solutions of the Falkner-Skan equation used in [80]:

$$
\begin{aligned}
f_{1}(H) & =\frac{\delta^{* 2}}{\nu} \frac{\partial V}{\partial x}=-2.4\left(1-e^{0.43\left(H_{0}-H\right)}\right) \quad \text { with } \quad H_{0}=2.59 \\
f_{2}(H) & =\frac{c_{f} \delta^{*} V}{2 \nu H}=\left(\frac{4}{H^{2}}-\frac{1}{H}\right) \\
f_{3}(H) & =\frac{\delta_{99}}{\delta^{*}}=\frac{H+2}{H-1}+\frac{4(H-4)(H-2.5)}{H^{3}} .
\end{aligned}
$$

The relation to flat plate flow is generally given by the shape factor which is defined as $H=\frac{\delta^{*}}{\theta}$, whereas $H_{0}$ is the equivalent value for plane flow over a flat plate. The curve fits provide a good approximation for values of $\mathrm{H}$ between 2 and 20 . At the separation point the wall shear stress vanishes, i.e. $\frac{\partial v_{x}}{\partial r}=0$, which is equivalent to a shape factor $H=4$. Relation (3.96) is not required for the calculations, however it is useful to predict the actual boundary layer thickness $\delta_{99}$, where the fluid velocity differs by $1 \%$ compared to the free stream value. The relation for the shear stress given in $[80]$ is

$$
\tau_{w}(x, t)=\frac{\mu f_{2} V}{\theta},
$$

consequently the friction factor reads

$$
c_{f}(x, t)=\frac{2 \mu f_{2}}{\rho_{0} V \theta} .
$$




\section{Viscous friction and momentum correction}

DEVELOPING flow conditions in ducts of multiply connected cross-sections generally make it difficult to use the right friction factor. A variety of cross-sections are discussed in [89]. In those situations the similarity parameters are preferably based on the free stream velocity and the square root of the cross-sectional flow area as characteristic length scale i.e. $\ell=\sqrt{A}$. In other words we have multiplied $R e$ and $S r$ by a factor of $\sqrt{\pi} / 2$, while $W_{O}$ is multiplied by $\sqrt{\pi}$. In the calculations we have given the Reynolds number inside the stenosis, $R e_{s t}$, based on $\ell$.

As previously mentioned the surface line of the flat portion of the non-circular duct dominates the circular portion at severe deformations, so that the computation of viscous forces is based on plane wedge flow. Consequently the thickness of the boundary layer is estimated in the $x z$-plane. Further we assume that the boundary layer has constant thickness along the circumference as illustrated in figure 3.10. The latter assumption allows a simple derivation of the momentum correction coefficient and the viscous friction term. Integration over the cross-section leads to geometric relations for the areas occupied by the displacement thickness $\delta^{*}$ and the total displacement thickness $\delta^{* *}$ in that cross-section. They are expressed as

$$
\begin{aligned}
A_{\delta^{*}} & =2 B \delta^{*}+\pi\left[R_{d}^{2}-\left(R_{d}-\delta^{*}\right)^{2}\right], \\
A_{\delta^{* *}} & =2 B \delta^{* *}+\pi\left[R_{d}^{2}-\left(R_{d}-\delta^{* *}\right)^{2}\right] .
\end{aligned}
$$

It is obvious that $A_{d}>A_{\delta^{* *}}$ and $A_{d}>A_{\delta^{*}}$ have to be satisfied to make sure that the flow is not fully developed. The momentum correction coefficient can be found by

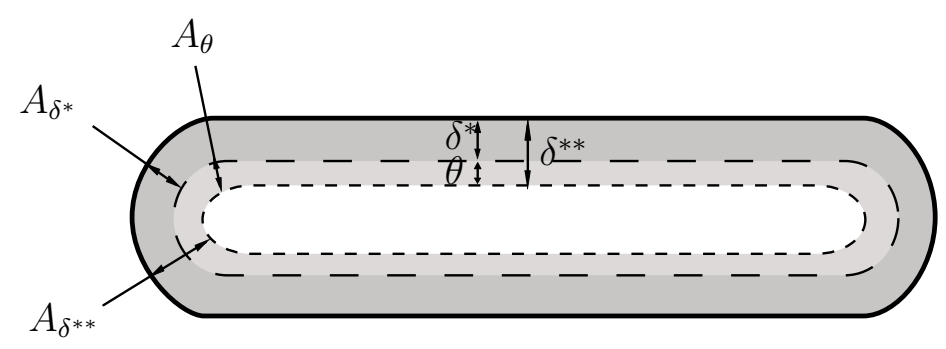

Figure 3.10: Illustration of the displacement thickness $\delta^{*}$, the momentum thickness $\theta$ and the total displacement thickness $\delta^{* *}$ in a deformed cross-section. The related areas are the displacement area $A_{\delta^{*}}$ (dark grey), the momentum area $A_{\theta}$ (light grey) and the total displacement area $A_{\delta^{* *}}=A_{\delta^{*}}+A_{\theta}$ (light + dark grey) respectively.

satisfying mass conservation for the mean flow and the core flow by

$$
V\left(A-A_{\delta^{*}}\right)=A u
$$


which can be used together with equation (3.84) and (3.100) in the definition for the momentum correction (3.25), so that

$$
\chi(x, t)=\frac{A}{\left(A-A_{\delta^{*}}\right)^{2}} \int_{A} \frac{v_{x}^{2}}{V^{2}} d a=A \frac{A-A_{\delta^{* *}}}{\left(A-A_{\delta^{*}}\right)^{2}}=\frac{1-\frac{A_{\delta^{* *}}}{A}}{\left(1-\frac{A_{\delta^{*}}}{A}\right)^{2}} .
$$

The uniform inflow profile is identical to $\chi=1$, while the developing profile reaches its far downstream value of 1.39 after the entrance length within less than $4.5 \%$ from the analytical solution for the parabolic flow profile given in equation (3.56). We note that in the linearised system the total cross-section $A$ in equation (3.102) is replaced by $A_{d}$. According to equation (3.98) the friction factor built with the pressure dependent circumference, $U_{p}(x, t)$ is

$$
F_{\nu}(x, t)=\frac{U_{p}}{\rho_{0}} \tau_{w}=-\frac{2 \nu\left(B+\pi R_{d}\right)}{\theta} f_{2} .
$$

Computations in a uniform tube show good agreement with the friction factor of the parabolic profile given in equation (3.55). After the entrance length the friction factor computed via the boundary layer theory reached its far downstream value to within $7 \%$. Additionally the Fanning friction factor Reynolds number product in a deformed vessel geometry agrees well with experiments carried out in [89]. In contrast to other proposed models the underlying model does not require knowledge about the fully developed friction factor Reynolds product $c_{f} R e_{f d}$ [89], or the incremental pressure drop factor $K_{\infty}$ [91, 93]. The above results for momentum correction and viscous friction quantify the entrance conditions typically encountered in studies of the arterial system.

\section{Boundary layer separation}

AT high Reynolds numbers, fluid particles within the boundary layer experience a momentum deficit relative to the core flow and are very susceptible to separation in regions of adverse pressure gradient. Solutions of the unsteady non-interactive boundary-layer equations are known to develop a generic separation singularity in regions where the pressure gradient is prescribed and adverse. For many years a solution was thought to be intractable by traditional techniques. However, in this study a solution was obtained by a strong viscous-inviscid interaction method [83, 84]. In the next paragraph we discuss the process of flow separation and reattachment using the proposed method.

BOUNDARY layers tend to separate from the vessel wall if the fluid pressure in direction of the flow increases, i.e. for adverse pressure gradient. Increasing the fluid pressure is similar to an increment in potential energy of the fluid and consequently reduced kinetic energy or deceleration of the flow. In those situations the boundary 
layer thickness increases, and the gradient of the velocity profile at the wall reduces, with simultaneous decrease in the wall shear stress. For a sufficiently large pressure gradient the shear stress is reduced to zero, and separation of the boundary layer may occur. The fluid is no longer bound to the wall, so that reverse flow regimes may develop further downstream, which effectively push the boundary layer away from the wall. As the boundary layer starts to separate from the surface, the pressure distribution in the core is altered through a geometric interaction between equation (3.71) and (3.101) just prior the formation of the separation singularity. This strong viscous-inviscid interaction of the boundary layer and the core flow avoids the development of the separation singularity. On the other hand, decreasing pressure gradients, which accelerate the fluid cause the boundary layer thickness to decrease. Favourable pressure gradients consequently increase the gradient of the velocity profile, i.e. recirculating regions thin out, so that the boundary layer reattaches.

A schematic illustration of the actual flow profile along the tube axis is shown in figure 3.11. In the case of accelerating flow the velocity profiles have no point of inflection, whereas in the case of decelerated flow they exhibit a point of inflection. We have chosen a uniform inflow profile with velocity $V(0, t)$. The boundary layer (dashed line) grows from the leading edge, decreases in the converging part, while it grows in the divergent part of the tube. The upward triangles $(\mathbf{\Delta})$ denote the point of separation, while downward triangles $(\boldsymbol{\nabla})$ indicate the reattachment of the boundary layer. After separation the flow field can be seen as a top hat profile in the centre and a recirculation zone close to the walls. Due to the adjacent converging part the reattachment is forced early because fluid is accelerated. In contrast the reattachment after the second diverging part takes place further downstream.

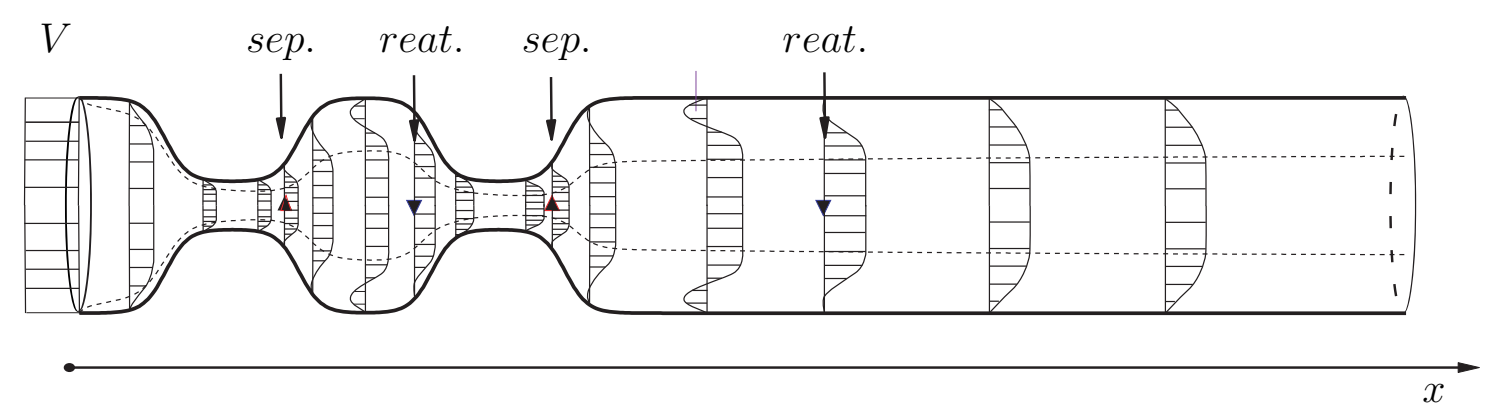

Figure 3.11: Illustration of boundary layer separation in a series of two myocardial bridges at a deformation of $\zeta_{0}=0.2$ (see 6.1); geometry and boundary layer thickness are displayed in realistic proportions, the velocity profiles are schematically drawn. The inflow profile is uniform with velocity $V$. We note that the extension of the separation zones differ, because the second myocardial bridge experiences different flow conditions. 
WE note that the boundary layer growth at the leading edge is of Blasius type, however due to continuity requirements, a retardation near the wall must cause the inviscid core to accelerate, thinning the boundary layer more than the Blasius estimate. Further, according to the derivation the system of equations is only valid for flow velocities with positive sign, which however is not the case in all parts of the cardiovascular system. Reverse flow can be found in several arteries, the coronary arteries fall into this category.

\subsection{Validity}

THE stationary boundary layer approximation becomes increasingly valid if the Reynolds number increases and when the ratio of unsteady forces to viscous forces given by the Womersley number is small. The approximation of the actual flow profiles by near equilibrium flow profiles is justified for stationary flow, however, nonstationary flow conditions require that the period $T$ of oscillation in equation (3.30) has to be large compared to the viscous diffusion time through the boundary layer: $t_{d}=\frac{\delta^{2}}{\nu} \ll T$. This is well satisfied for the situation under consideration, where $t_{d}$ in the centre of the deformation is between $4.4 * 10^{-3} \mathrm{~s}$ and $0.2 \mathrm{~s}$ at values of $\zeta_{0}$ of 0.25 and 1 respectively. Yet another indication that quasi-stationary flow profiles are sufficient is given in [129]. It was found that for small oscillatory perturbations the system can be assumed to be linear, i.e. no appreciable variations in long-term averages are observed in non-stationary flow conditions and otherwise that sinusoidal perturbation always causes sinusoidal system response. The nonlinear non-stationary effects become important when the fraction of non-stationary to stationary flow velocity exceeds $60 \%$. Even so the variation of mean wall shear stress is below $4 \%$ and consequently the variations in flow resistance. In other words as their is no flow reversal those effects can be justifiably neglected, i.e. quasi-stationary flow profiles are sufficient to represent the mean wall shear stress if amplitude and frequency are small. However we note that the application of time dependent similarity solutions may enhance the accuracy in flow situations where transient inertial forces dominate. We further note that the method is simply modified to simulate turbulent flow conditions.

ANOTHER point that requires verification is that the accumulated net flow caused by the compression of the artery is identical to the change in vessel volume caused by the compression. For this purpose we have assumed that no volume enters the tube by an inflow condition and that the net flow caused by the deformation may simply pass the boundary. In such a situation the net flow across both boundaries should equal zero after a temporal integration over subsequent compression and relaxation of the tube. The results for an integration including $4 s$ after vessel relaxation have indicated that at either boundary the net flow is preserved by less than $0.21 \%$ of the total tube volume. 


\section{Chapter 4}

\section{Theoretical Aspects}

Tn this chapter we discuss the basic properties of the governing equations for one-

1 dimensional approximation of blood flow in arteries. Firstly we address the hyperbolicity and non-linearity of the equations in physiologic flow conditions. Since the averaged flow equations (3.27a) and (3.27b) are in conservative form the second part of this chapter discusses the conditions under which the equations may be expressed by characteristic equations. These considerations are necessary since they allow for the development of approximate Riemann solvers. Riemann problems arise from discretising discontinuities in cross-section and elasticity of the vessels and in arterial bifurcations. The chapter finishes with boundary and interface conditions of vascular networks and physiological conditions under which the considered model can give reasonable results.

\subsection{Hyperbolicity and non-linearity}

A variety of theoretical studies concerning non-linearity and hyperbolicity of the one-dimensional arterial model are found in the literature [130, 48, 20, 131]. In those each vessel of the arterial network is described by a nonlinear boundary value problem, where the boundary values arise from different physiological situations, as described in chapter 2. For reasons that the equations are quasi-linear, it is not clear a priori that the system is always hyperbolic and has a bounded solution. It was found that reasonable conditions to satisfy hyperbolicity are (i) that the arterial cross-section remains positive and (ii) that the flow velocity satisfies $v \ll c$ [48]. These conditions are generally present in healthy individuals, where the arterial cross-section of the vessel is always positive and the speed at which pulse waves propagate is much larger than the fluid velocity. In contrast vascular diseases like arterial stenosis, aneurisms, arteriosclerosis may alter the conditions considerably, so that total occlusion (crosssection equals zero) or vessel collapse $(u>c)$ may occur. Further, it was shown that 
pulsatile flow conditions in semi-finite compliant vessels give rise to shock formation, but that the first shock formation is well outside the physiological domain [48].

THE non-linearities of the equations were further discussed in [130]. Possible sources of non-linearities are (i) convective inertia forces due to the term $u \frac{\partial u}{\partial x}$, which become important if the vessel geometry changes in axial direction, (ii) the viscous friction term $\mu \nabla^{2} u$, if the fluid viscosity is dependent on shear rate, (iii) the wall elasticity and finally (iv) non-linearities in geometry as they appear in deformed tubes.

\subsection{Characteristic system}

ONE property of hyperbolic equations is the existence of characteristic curves on which the partial differential equation reduces to an ordinary differential equation. The aim of the method of characteristics is to find curves in the $x-t$ plane (plane of independent variables), on which the characteristic variables propagate. These variables are also known as Riemann invariants. The procedure is exact as long as the solution is smooth and differentiable. A detailed description of this method can be found in $[132,133]$. The following derivation is motivated by $[49,130,22,23]$

THE equations (3.27a) and (3.27b) can be written in a pair of quasi-linear hyperbolic partial differential equations in the two dependent variables $A$ and $u$. The conservation law is of the form

$$
\frac{\partial \mathbf{U}}{\partial t}+\mathbf{M}(\mathbf{U}) \frac{\partial \mathbf{U}}{\partial x}=\left[\begin{array}{l}
A \\
u
\end{array}\right]_{t}+\left[\begin{array}{cc}
u & A \\
\frac{1}{\rho_{0} \mathcal{D}_{A} A} & u
\end{array}\right]\left[\begin{array}{l}
A \\
u
\end{array}\right]_{x}=\left[\begin{array}{l}
0 \\
f
\end{array}\right]
$$

whereas the forcing term is given by

$$
f=-\frac{1}{\rho_{0}}\left(\frac{\partial p}{\partial \zeta} \frac{\partial \zeta}{\partial x}+\frac{\partial p}{\partial E} \frac{\partial E}{\partial x}\right)+\frac{F_{\nu} u}{A} .
$$

We assume that the gradients $\frac{\partial E}{\partial x}$ and $\frac{\partial \zeta}{\partial x}$ are zero on the boundary, i.e. there is no change in elastic properties and the vessel geometry is uniform. Under the assumption that $A>0$ the matrix $\mathbf{M}$ has two real eigenvalues $\lambda^{ \pm}=u \pm c$,

$$
\lambda(\mathbf{M})=\left[\begin{array}{l}
u+\frac{1}{\sqrt{\rho_{0} \mathcal{D}_{A}}} \\
u-\frac{1}{\sqrt{\rho_{0} \mathcal{D}_{A}}}
\end{array}\right],
$$

the corresponding left eigenvectors of $\mathbf{M}(\mathbf{U})$ are

$$
\mathbf{L}(\mathbf{M})=\left[\begin{array}{c}
A \sqrt{\rho_{0} \mathcal{D}_{A}}-A \sqrt{\rho_{0} \mathcal{D}_{A}} \\
1 \\
1
\end{array}\right]=\left[\begin{array}{c}
\frac{\partial w^{+}}{\partial A} \frac{\partial w^{-}}{\partial A} \\
\frac{\partial w^{+}}{\partial u} \frac{\partial w^{-}}{\partial u}
\end{array}\right],
$$

and $\mathbf{M}=\mathbf{L} \boldsymbol{\Lambda} \mathbf{L}^{-1}$ with

$$
\mathbf{\Lambda}(\mathbf{M})=\left[\begin{array}{cc}
\lambda^{+} & 0 \\
0 & \lambda^{-}
\end{array}\right]
$$


the diagonal eigenvalue matrix. Pre-multiplication of equation (4.1) by $\mathbf{L}$ and subsequent change in variables by $\frac{\partial \mathbf{W}}{\partial \mathbf{U}}=\mathbf{L}$, transforms the above system into the characteristic equations

$$
\frac{\partial \mathbf{W}}{\partial t}+\Lambda \frac{\partial \mathbf{W}}{\partial x}=\frac{1}{2} f
$$

The vector of characteristics in the above system is given by $\mathbf{W}=\left[\omega^{+}, \omega^{-}\right]^{T}$. The characteristic variables defined as $\frac{d x^{ \pm}}{d t}=w^{ \pm}$, are unknown functions and $\Lambda$ are smooth functions of $w^{ \pm}$. Emanate from the eigenvalues is that $\lambda^{+}>\lambda^{-}$, i.e. the characteristics of the system are discriminative and have opposite directions, which is indicated by the \pm signs. Under prior assumptions the system is strictly hyperbolic and the wave speed of the nonlinear system, $c_{A}=\frac{1}{\sqrt{\rho_{0} \mathcal{D}_{A}}}$, is always positive and generally much larger than the velocity of blood. The characteristic variables are found by the integration of $\frac{\partial \mathbf{W}}{\partial \mathbf{U}}=\mathbf{L}(\mathbf{M})$, i.e. that $\frac{\partial w^{ \pm}}{\partial u}=1$ and $\frac{\partial w^{ \pm}}{\partial A}= \pm A \sqrt{\rho_{0} \mathcal{D}_{A}}$. The explicit relation for the characteristic variables is obtained by subsequent introduction of the wave speed, so that

$$
\begin{aligned}
w^{ \pm}(x, t) & =\int_{u_{d}}^{u} d u \pm \int_{A_{d}}^{A} \frac{1}{A \sqrt{\rho_{0} \mathcal{D}_{A}}} d A= \\
u & -u_{d} \pm 2 \frac{\sqrt{\rho_{0} \mathcal{D}_{A_{d}}}-\sqrt{\rho_{0} \mathcal{D}_{A}}}{\sqrt{\rho_{0} \mathcal{D}_{A_{d}}} \sqrt{\rho_{0} \mathcal{D}_{A}}}=u-u_{d} \pm 2\left(c_{A}-c_{A_{d}}\right)
\end{aligned}
$$

where $c_{A d}$ is the wave speed of the linearised system. The equilibrium condition $\left(u_{d}, A_{d}\right)$ suggests that $u_{d}=0$ when $A=A_{d}$. A system of linearised equations can be written by introduction of perturbation variables $A^{\prime}$ and $u^{\prime}$. The distensibility for the equilibrium condition is

$$
\mathcal{D}_{A_{d}}=\frac{2 R_{d}}{A_{d}} \frac{\partial L(\zeta, p)}{\partial p}=\frac{U_{0} R_{d}^{2}}{A_{d}} \frac{\left(1-\sigma^{2}\right)}{E h_{0}},
$$

and the pressure-area relationship in equation (3.48) reduces to

$$
p\left(R_{d}, A^{\prime}\right)=\frac{E h_{0}}{\left(1-\sigma^{2}\right)} \frac{A^{\prime}}{U_{0} R_{d}^{2}} .
$$

The linearised system can be written as

$$
\frac{\partial \mathbf{U}_{d}}{\partial t}+\mathbf{M}_{d}\left(\mathbf{U}_{d}\right) \frac{\partial \mathbf{U}_{d}}{\partial x}=\left[\begin{array}{l}
A^{\prime} \\
u^{\prime}
\end{array}\right]_{t}+\left[\begin{array}{cc}
0 & A_{d} \\
\frac{1}{\rho_{0} \mathcal{D}_{A_{d}} A_{d}} & 0
\end{array}\right]\left[\begin{array}{l}
A \\
u^{\prime}
\end{array}\right]_{x}=\left[\begin{array}{l}
0 \\
f
\end{array}\right]
$$

with the following eigenvalues and eigenvectors of $\mathbf{M}_{d}\left(\mathbf{U}_{d}\right)$

$$
\lambda_{d}\left(\mathbf{M}_{d}\right)=\left[\begin{array}{c}
\frac{1}{\sqrt{\rho_{0} \mathcal{D}_{A_{d}}}} \\
-\frac{1}{\sqrt{\rho_{0} \mathcal{D}_{A_{d}}}}
\end{array}\right], \quad \mathbf{L}_{d}\left(\mathbf{M}_{d}\right)=\left[\begin{array}{c}
A_{d} \sqrt{\rho_{0} \mathcal{D}_{A_{d}}}-A_{d} \sqrt{\rho_{0} \mathcal{D}_{A_{d}}} \\
1 \\
1
\end{array}\right]=\left[\begin{array}{l}
\frac{\partial w^{+}}{\partial A_{d}} \frac{\partial w^{-}}{\partial A_{d}} \\
\frac{\partial w^{+}}{\partial u^{\prime}} \frac{\partial w^{\prime}}{\partial u^{\prime}}
\end{array}\right] .
$$


The characteristic equations for the linearised equations are found to be identical to those of the non-linear equations, however, the characteristic curves, along which the characteristic equations apply, are different.

$$
w^{ \pm}(x, t)=u^{\prime} \pm \int_{A_{d}}^{A} \frac{1}{A_{d} \sqrt{\rho_{0} \mathcal{D}_{A_{d}}}} d A=u^{\prime} \pm \frac{A^{\prime}}{A_{d} \sqrt{\rho_{0} \mathcal{D}_{A_{d}}}}=u^{\prime} \pm c_{A_{d}} \frac{A^{\prime}}{A_{d}} .
$$

Here $c_{A_{d}}=\frac{1}{\sqrt{\rho_{0} \mathcal{D}_{A_{d}} A_{d}}}$ is the wave speed for the linearised system. Linearisation of the equations therefore neglects the fluid velocity in the expression of the characteristic lines. Both systems are hyperbolic and subcritical, so that we require one boundary condition at each end of the tube. They are implemented through the characteristic system and use the following compatibility relations

$$
A^{\prime}=\frac{A_{d}}{c_{A_{d}}} \frac{\left(w^{+}-w^{-}\right)}{2}, \quad u^{\prime}=\frac{\left(w^{+}+w^{-}\right)}{2}
$$

for the linear system as initial guess to solve the nonlinear in- and outflow conditions.

\subsection{Interface and boundary conditions}

As mentioned earlier the human cardiovascular system is a closed system of tubular entities. Parameters and exact course of the major 175 arteries are found in table A.1 and figure A.1 in the appendix. The description as separate segments requires interface conditions at the bifurcations and physiological boundary conditions at places where the computational domain is terminated, i.e. in- and outflow conditions. These conditions are based on physical laws and physiological factors respectively. The implementation is based on the characteristic system. In the following section we will shortly discuss common conditions used in cardiovascular system dynamics.

\subsubsection{Approximation of boundary characteristics}

DIFFERENT types of in/outflow conditions for the characteristics may be applied. Non-reflecting boundary conditions are useful to approximate the characteristics at the boundary. They are frequently implemented by one-way wave equations, which for the forward and backward travelling characteristics are given in (4.14) and (4.15) respectively.

$$
\begin{aligned}
& \left(\frac{\partial}{\partial x}+\frac{1}{\lambda^{+}} \frac{\partial}{\partial t}\right) w^{+}(x=L, t+\delta t)=\left(\frac{\partial}{\partial x}+\frac{1}{\lambda^{+}} \frac{\partial}{\partial t}\right) w^{+}(x=L-\delta x, t) \\
& \left(\frac{\partial}{\partial x}+\frac{1}{\lambda^{-}} \frac{\partial}{\partial t}\right) w^{-}(x=0, t+\delta t)=\left(\frac{\partial}{\partial x}+\frac{1}{\lambda^{-}} \frac{\partial}{\partial t}\right) w^{-}(x=0+\delta x, t)
\end{aligned}
$$


This, however, assumes that the primary wave direction is normal to the boundary and that none of the discriminative characteristics

$$
\begin{array}{ll}
w^{+}(x=0, t)=0 & \text { (no right travelling wave), } \\
w^{-}(x=L, t)=0 & \text { (no left travelling wave), }
\end{array}
$$

enter the domain. We note that wave reflections will occur as those conditions are not satisfied. The above equations are used to implement both the in/outflow and branching conditions. The method of characteristics is used to approximate the characteristic variables $w^{+}$and $w^{-}$at the boundary at $x=0$ and $x=L$. As illustrated in figure $4.1, w^{+}$characteristics leave the domain at $x=L$, while $w^{-}$characteristics leave the domain at $x=0$ as time increases.

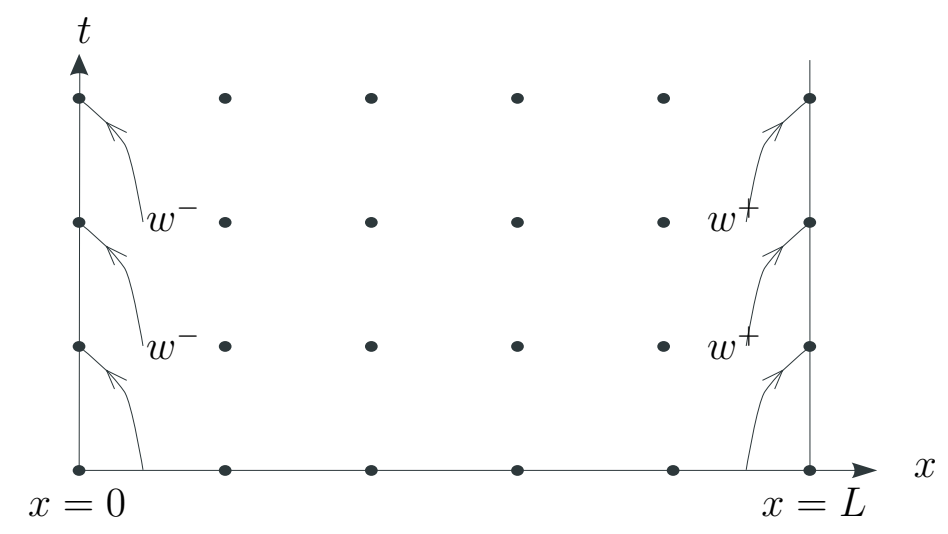

Figure 4.1: Plane of independent variables. Approximation of boundary characteristics by one-way wave equations. As time increases positive characteristics $w^{+}$leave the domain at $x=L$, while negative characteristics $w^{-}$leave the domain at $x=0$.

\subsubsection{Inflow boundary conditions}

THE inflow boundary condition at the aorta is given by the flow velocity, the area, or a relation between them. The shape of the pulse wave in the ascending aorta is mainly generated by the aortic valve. The pressure-flow conditions are known from in vivo measurements, so that either a periodic extension of a measured flow wave or a synthesised wave in the exponential form

$$
q_{i n}(t)=q_{0}\left[\frac{t}{t_{r}} \exp ^{\frac{-t^{2}}{2 t_{r}^{2}}}+\frac{2 t}{t_{r}} \exp ^{\frac{-t^{2}}{t_{r}^{2}}}\right]
$$

is used. Here the amplitude of the exponential waveform is $q_{0}$, while $t_{r}$ is the rising time. For reasons that the coronary flow is primarily driven by the aortic pressure, 
the pulsatile boundary condition to the left main coronary artery was represented by a periodic extension to a synthetic pressure wave in the exponential form

$$
p_{i n}(t)=p_{s}+p_{0} \frac{t}{t_{r}} \exp ^{\frac{-t^{2}}{2 t_{r}^{2}}}
$$

where $p_{s}$ is the static pressure and $p_{0}$ is the amplitude of the exponential waveform. The parameters used in the simulations are specified later in the text.

\subsubsection{Interface conditions}

THE branching conditions for a bifurcation shown in figure 4.2 are physically motivated by the conservation of mass and the requirement of constant pressure at the branching point [23]. The subscripts $p, d_{1}$ and $d_{2}$ stand for the parent, $1^{\text {st }}$ and $2^{\text {nd }}$ daughter vessels respectively. The mass flux balance is $q_{p}=q_{d 1}+q_{d 2}$, while the en-

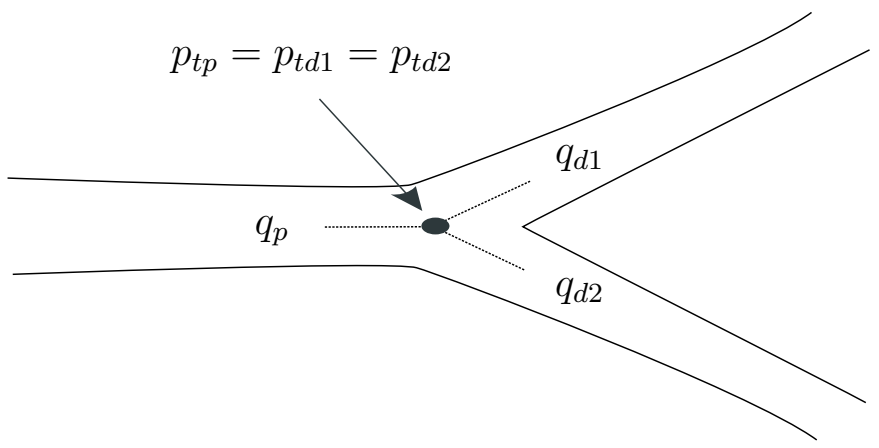

Figure 4.2: Flow and pressure in a vascular bifurcation. The subscripts $p, d_{1}$ and $d_{2}$ stand for the parent, $1^{\text {st }}$ and $2^{\text {nd }}$ daughter vessels respectively.

ergy conservation leads to continuity of total pressure $p_{t}$, i.e. $p_{t p}=p_{t d 1}=p_{t d 2}$. The interface conditions for the nonlinear system are therefore

$$
\begin{aligned}
A_{p} u_{p} & =A_{d_{1}} u_{d_{1}}+A_{d_{2}} u_{d_{2}} \\
p_{p}+\frac{1}{2} \rho_{0} u_{p}^{2} & =p_{d_{1}}+\frac{1}{2} \rho_{0} u_{d_{1}}^{2}, \\
p_{p}+\frac{1}{2} \rho_{0} u_{p}^{2} & =p_{d_{2}}+\frac{1}{2} \rho_{0} u_{d_{2}}^{2}
\end{aligned}
$$

while the linear system has similar conditions except that the static pressure is continuous across the bifurcation [23]. 


\subsubsection{Outflow boundary conditions}

THERE are several ways to account for peripheral reflections at the terminals starting from pure resistive load, where the outflow is proportional to the pressure over three and four-element windkessel models [14, 57] to a structured tree (fractal) outflow condition suggested in [59]. In the current simulations we have implemented a three-element windkessel model for the termination of the arterial network [25, 64]. The main advantage of this model is to consider the compliant-capacitive effects due to micro-vessels and arterioles. The lumped analogue electrical circuit is shown in

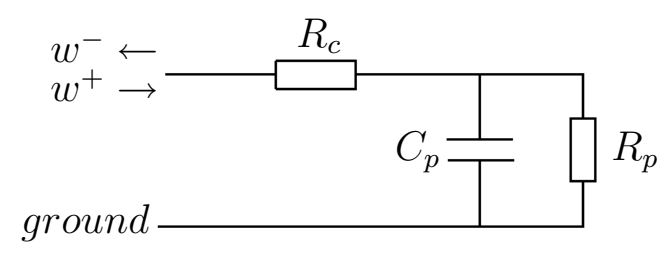

Figure 4.3: Three-element windkessel analogue circuit.

figure 4.3. According to [64] the differential equation in the time domain satisfied by the circuit is

$$
\frac{\partial p}{\partial t}=R_{c} \frac{\partial q}{\partial t}-\frac{p}{R_{p} C_{p}}+\frac{q\left(R_{p}+R_{c}\right)}{R_{p} C_{p}}
$$

with $R_{p}$ and $C_{p}$ being the peripheral resistance and compliance respectively. However $R_{c}$ is the characteristic impedance of the terminating vessel, which for large vessels is a real number and modelled by a resistor. The total peripheral resistance $R_{t}=R_{p}+R_{c}$ for each of the terminals was estimated by the total arterial peripheral resistance and the distribution of flow through the various branches [122]. The ratio $R_{c} / R_{t}$ was estimated in [134] by fit to data and found to be approximately 0.2. Finally, the arterial compliance $C_{p}$ for each segment was estimated from the total volume compliance [26]. Parameter values for $R_{t}$ and $C_{p}$ found in [20, 122] are listed in table A.1 in the appendix. 


\section{Chapter 5}

\section{Numerical Aspects}

S

EVERAL numerical challenges must be overcome before an adequate solution to afore mentioned equations is obtained. Not only the complex dynamical behaviour and non-linearity of the system but also the difficulties in boundary and interface conditions of arterial networks have to be addressed. Due to the non-linear terms in equation (3.85) and (3.27b) the solutions for haemodynamically developing flows are generally more difficult to obtain than fully developed flows or oscillating flows with a frequency dependent Stokes boundary layer. Developing flows require simultaneous solution of the continuity equation (3.27a), the momentum equation (3.27b) and the integral momentum equation (3.85), together with the boundary conditions given in (4.18), (4.20 - 4.23) and (3.86). The momentum correction factor and the viscous friction in equation (3.27b) are given by the solution to the integral momentum equation (3.85) and the two curve fits to the Falkner-Skan equation in (3.94) and (3.95), i.e. through the solution of equation (3.102) and (3.103). A structogram of the algorithm can be found in figure 5.4 at the end of this chapter .

\subsection{Choice of numerical method}

A variety of numerical schemes were used in blood flow simulations. Besides finite difference and finite volumes schemes there is the branch of spectral methods and the method of characteristics. Finite difference schemes are constructed by replacing continuous derivatives with appropriate finite difference approximations defined by a set of discrete points. Further distinction is drawn between up/downwind and central differences. The oscillatory nature of (non-linear) hyperbolic equations is known to raise high frequency oscillations (with wavelength $2 d x$ ), when centrally differenced, because the value of the slope estimated is independent of the value of the dependent variable at the mid-point. Those oscillations can be reduced by upwind methods, because disturbances in the flow regime that affect convective acceleration can not 
be propagated upstream. However they are generally only first-order accurate and in practical applications, the MacCormack scheme for the solution of the non-linear equations is recommended. Detailed comparison of finite difference schemes for nonlinear differential equations is presented in [102]. Higher order corrected schemes can be found in [103].

FOR simplicity we have chosen an explicit second-order MacCormack predictorcorrector method as the basic scheme to discretise the set of equations. The two-step algorithm contains a small amount of both numerical diffusion and dispersion, which makes it numerically stable. More importantly this scheme is non-linearly stable, and has been successfully used to solve similar equations [102]. Besides the ability to treat interface and boundary conditions of the arterial network the method is able to resolve regions where strong, sharp gradients exist.

Throughout this chapter we use a regular grid with uniform temporal and spatial grid intervals. In this way consistency is automatically achieved, while stability is not guaranteed. The stability of linear hyperbolic equations discretised by explicit finite difference methods is generally given by the $C F L$ condition (Courant, Friedrich, Levy, 1928). However, the averaged flow equations are non-linear, and a rigourous stability analysis for these equations is exceedingly difficult. Therefore, the $C F L$ condition can only be considered as a general guideline here, and the maximum allowable time step (with respect to stability) for any particular problem will vary about the predicted value and is determined by numerical experimentation. All simulations where performed under those criterions and are found to be valid in the region of interest.

\subsubsection{Discretisation of the averaged flow equations}

Starting from the initial conditions at time level $(n)$, the flow variables at the interior nodes are predicted from the initial boundary condition in equation (4.18) or (4.19) by a backward difference in space, while a forward difference in space of the predicted variables is used in the corrector step. The direction of both prediction and correction is alternated at each time step (wind averaging). The first prediction step was chosen in the direction of wave propagation, because the scheme produces better results. To calculate future values at $(n+1)$ the scheme requires values at $(n)$. In the following discretisation the subscript $i$ indicates the nodes along the axis of each segment, $m$, and the superscript $n$ refers to the time level, i.e. the spatial grid in axial direction is given as $0 \leq i \leq I$ with step width $d x$, and the grid over time is $0 \leq n \leq N$ with step width $d t$. The variables $u^{\bullet}, A^{\bullet}$ and $p^{\bullet}$ are intermediate predicted values of $u, A$ and $p$ respectively. The corrector step provides the final value of $u, A$ and $p$ at time $(n+1)$. The discrete spatial operators for the predictor step for even time steps $(n \bmod 2=0)$ can be written as

$$
\frac{d q}{d x}=\frac{q_{i+1}^{n}-q_{i}^{n}}{d x}
$$




$$
\begin{aligned}
\frac{d p}{d x} & =\frac{p_{i+1}^{n}-p_{i}^{n}}{d x}, \\
\frac{d(\chi u)}{d x} & =\frac{(\chi u)_{i+1}^{n}-(\chi u)_{i}^{n}}{d x},
\end{aligned}
$$

while for odd time steps $(n \bmod 2 \neq 0)$ they are

$$
\begin{aligned}
\frac{d q}{d x} & =\frac{q_{i}^{n}-q_{i-1}^{n}}{d x}, \\
\frac{d p}{d x} & =\frac{p_{i}^{n}-p_{i-1}^{n}}{d x}, \\
\frac{d(\chi u)}{d x} & =\frac{(\chi u)_{i}^{n}-(\chi u)_{i-1}^{n}}{d x} .
\end{aligned}
$$

Discretisation of mass and momentum conservation with an Euler step yields

$$
\begin{aligned}
\left(A^{\prime}\right)_{i}^{\bullet} & =\left(A^{\prime}\right)_{i}^{n}-d t \frac{d q}{d x}-\left[\left(A_{d}\right)_{i}^{n+1}-\left(A_{d}\right)_{i}^{n}\right], \\
u_{i}^{\bullet} & =u_{i}^{n}-d t\left[\left(\frac{u(\chi-1)}{A}\right)_{i}^{n} \frac{d q}{d x}+u \frac{d(\chi u)}{d x}+\frac{1}{\rho_{0}} \frac{d p}{d x}-\left(\frac{F_{\nu}}{A}\right)_{i}^{n}\right] .
\end{aligned}
$$

The discretised spatial derivatives of the corrector step for even time steps $(n \bmod 2=$ $0)$ are given by

$$
\begin{aligned}
\frac{d q^{\bullet}}{d x} & =\frac{\left(q^{\bullet}\right)_{i}^{n}-\left(q^{\bullet}\right)_{i-1}^{n}}{d x}, \\
\frac{d p^{\bullet}}{d x} & =\frac{\left(p^{\bullet}\right)_{i}^{n}-\left(p^{\bullet}\right)_{i-1}^{n}}{d x}, \\
\frac{d\left(\chi u^{\bullet}\right)}{d x} & =\frac{\left(\chi u^{\bullet}\right)_{i}^{n}-\left(\chi u^{\bullet}\right)_{i-1}^{n}}{d x},
\end{aligned}
$$

while for odd time steps $(n \bmod 2 \neq 0)$ they are

$$
\begin{aligned}
\frac{d q^{\bullet}}{d x} & =\frac{\left(q^{\bullet}\right)_{i+1}^{n}-\left(q^{\bullet}\right)_{i}^{n}}{d x}, \\
\frac{d p^{\bullet}}{d x} & =\frac{\left(p^{\bullet}\right)_{i+1}^{n}-\left(p^{\bullet}\right)_{i}^{n}}{d x}, \\
\frac{d\left(\chi u^{\bullet}\right)}{d x} & =\frac{\left(\chi u^{\bullet}\right)_{i+1}^{n}-\left(\chi u^{\bullet}\right)_{i}^{n}}{d x} .
\end{aligned}
$$

Discretisation of mass and momentum conservation in the corrector step of the MacCormack scheme is accoring to [102]

$$
\begin{aligned}
\left(A^{\prime}\right)_{i}^{n+1} & =\frac{1}{2}\left\{\left(A^{\prime}+A^{\prime \bullet}\right)_{i}^{n}-d t \frac{d q^{\bullet}}{d x}\right\}-\left[\left(A_{d}\right)_{i}^{n+1}-\left(A_{d}\right)_{i}^{n}\right] \\
u_{i}^{n+1} & =\frac{1}{2}\left\{\left(u+u^{\bullet}\right)_{i}^{n}-d t\left[\left(\frac{u^{\bullet}(\chi-1)}{A^{\bullet}}\right)_{i}^{n} \frac{d q^{\bullet}}{d x}+u \frac{d\left(\chi u^{\bullet}\right)}{d x}+\frac{1}{\rho_{0}} \frac{d p^{\bullet}}{d x}-\left(\frac{F_{\nu}}{A^{\bullet}}\right)_{i}^{n}\right]\right\}
\end{aligned}
$$


Care must be taken while discretising squared variables, as they appear in conservative form (i.e. as in equation (3.24a) and (3.24b)), because the finite difference equation in conservative form loses information about the sign of the velocity. This is called weak numerical instability. The difficulty is solved by replacing $\left(q^{2}\right)_{i+1}^{n}-\left(q^{2}\right)_{i}^{n}$ by $0.5\left(q_{i+1}^{n}+q_{i}^{n}\right)\left(q_{i+1}^{n}-q_{i}^{n}\right)$ and $\left(q^{2}\right)_{i}^{n}-\left(q^{2}\right)_{i-1}^{n}$ by $0.5\left(q_{i}^{n}+q_{i-1}^{n}\right)\left(q_{i}^{n}-q_{i-1}^{n}\right)$ [102].
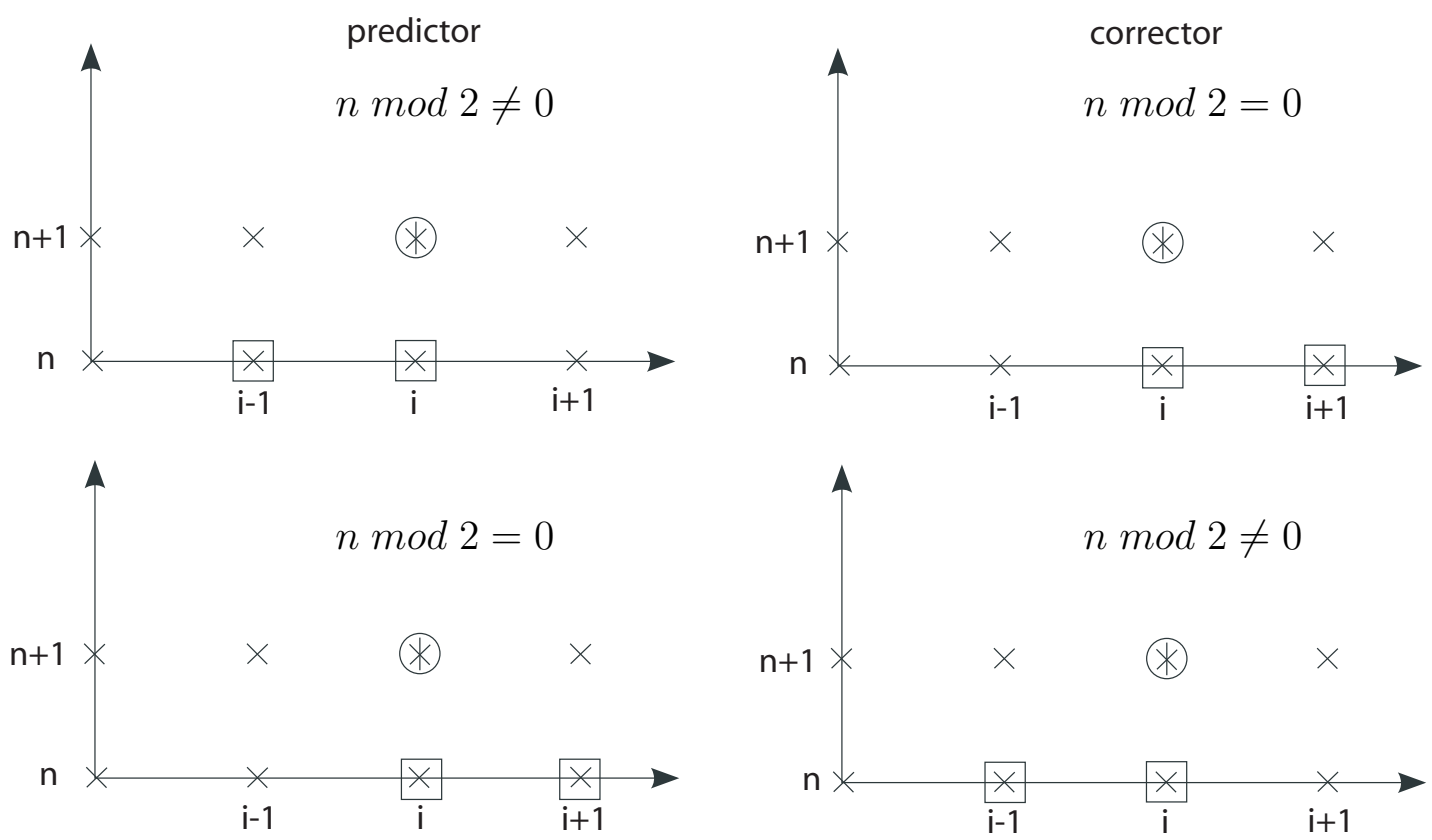

Figure 5.1: Stencils for the wind averaging MacCormack scheme. Predictor and corrector stencils are given for even $(n \bmod 2=0)$ and odd time steps $(n \bmod 2 \neq 0)$. Desired values at $n+1$ are illustrated as $\circledast$, required values for the computation are illustrated as $\otimes$.

We note that the averaged flow conditions are solved for all vessels in the network, before the boundary layer properties are determined. This is possible, because the averaged flow equations are only dependent on prior values of momentum correction factor and the viscous friction.

\subsubsection{Discretisation of the von Kármán momentum equation}

THE integral boundary layer equation (3.85) is discretised by a first order upwind scheme, the resulting discrete equations are iteratively solved for $\delta^{*}$. The spatial derivatives are

$$
\frac{d \theta}{d x}=\frac{\left(\frac{\delta^{*}}{H}\right)_{i}^{n}-\theta_{i-1}^{n}}{d x}
$$




$$
\begin{aligned}
\frac{d V}{d x} & =\frac{V_{i}^{n}-V_{i-1}^{n}}{d x} \\
\frac{d r_{w}^{j}}{d x} & =\frac{\left(r_{w}^{j}\right)_{i}^{n}-\left(r_{w}^{j}\right)_{i-1}^{n}}{d x}
\end{aligned}
$$

The discretisation of the stationary and transient integral momentum equation is

$$
\begin{aligned}
0 & =\left(\delta^{*} \frac{V}{\nu}\right)_{i} \frac{d \theta}{d x}+\left(\delta^{* 2} \frac{V}{\nu H r_{w}^{j}}\right)_{i} \frac{d r_{w}^{j}}{d x}+\left(\left(1+\frac{2}{H}\right) f_{1}-v_{w} \frac{\delta^{*}}{\nu}-H * f_{2}\right)_{i}, \\
\left(\delta^{*}\right)_{i}^{n+1} & =\frac{\left(V \delta^{*}\right)_{i}^{n}}{V_{i}^{n+1}}-\frac{\left(V^{2}\right)_{i}^{n}}{V_{i}^{n+1}}\left[\frac{d \theta}{d x}+\left(\frac{2 \theta+\delta^{*}}{V}\right)_{i}^{n} \frac{d V}{d x}+\left(\frac{\theta}{r_{w}^{j}}\right)_{i}^{n} \frac{d r_{w}^{j}}{d x}-\left(\frac{v_{w}}{V}-\frac{c_{f}}{2}\right)_{i}^{n}\right] d t .(5.6
\end{aligned}
$$

We note that the closure relations in equation (3.94) and (3.95) restrict the rage of validity, firstly because $\frac{1}{2.4} \frac{\delta^{*}}{\nu} \frac{\partial V}{\partial x}>-1$ has to be satisfied in the former relation and secondly due to the singularity at $H=1,0$ in the latter relation. The interval of solutions for $\delta^{*}$ therefore is restricted to the range $2<H<20$, where the curve fits to the Falkner-Skan equation provide good approximations. Because the NewtonRaphson iteration is inappropriate for this type of problem the solution is obtained by a combined root bracketing, interval bisection and inverse quadratic interpolation method of van Wijngaarden-Brent-Dekker. Detailed description of the method can be found in [135]. The confining upper and lower brackets of $\delta^{*}$ required by the algorithm are set by solving equation (3.94) with an adaptive forward and backward iteration until either $H \approx 2$ and $H \approx 20$ or the above restriction is reached.

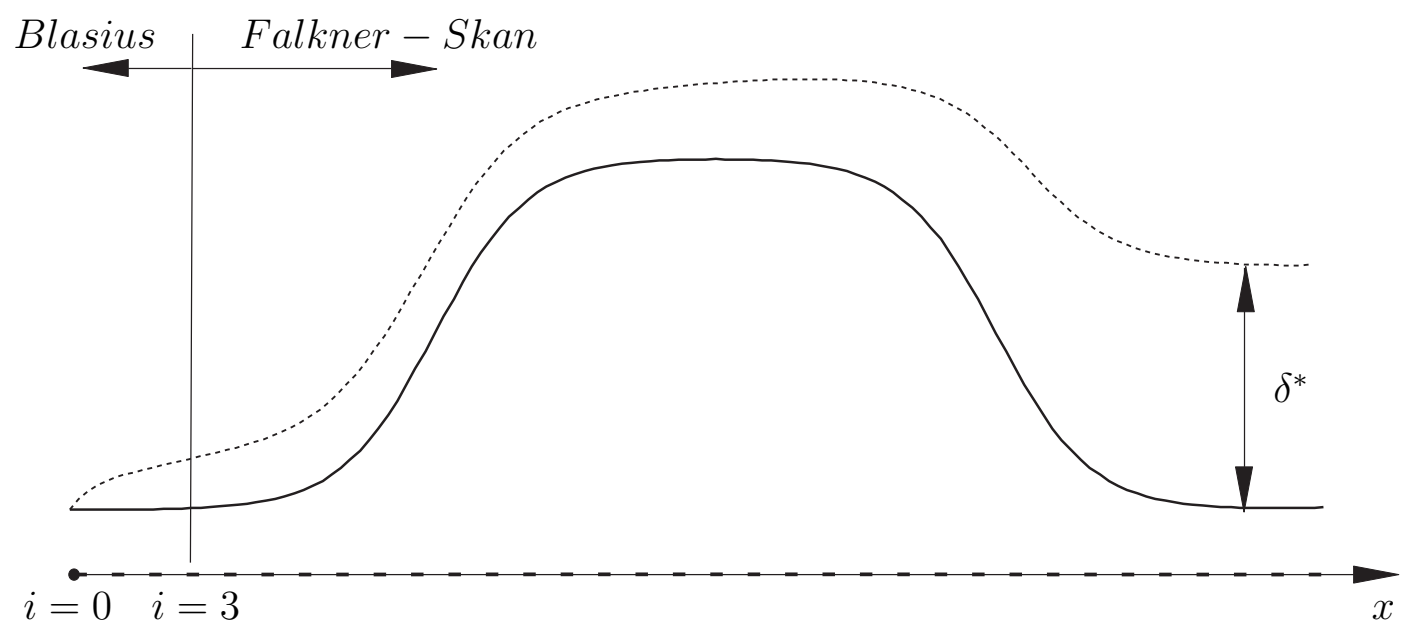

Figure 5.2: The boundary condition in equation (3.86) is prescribed a few grid points downstream (at $i=3$ ) by computing the Blasuis solution for $i \leq 3$. Downstream marching of equation (3.85) leads to values of $\delta^{*}$ at grid points $i>3$. The thick solid line indicates the curvature of the vessel wall, while the boundary layer is illustrated by a dotted line. 
THE entrance boundary condition in (3.86) is satisfied by the Blasius solution, which was shown to be the leading order term [80]. The computation is started a few grid points downstream from the inlet, where the Blasius solution provides values of the boundary layer thickness at each time step (see figure 5.2). We note that the vessel has to be uniform at the transition location Blasius/Falkner-Skan. The solution was applied to the steady integral momentum equation as boundary condition for $\delta^{*}$. Downstream marching the solution by the above method leads to the values of boundary properties along the tube axis. Through equation (3.102) and (3.103) one obtains the required values for momentum correction and viscous friction in the averaged flow equations (3.27a) and (3.27b).

\subsection{Numerical treatment of boundary conditions}

To implement the boundary and interface conditions it is convenient to neglect viscous friction. As described in the previous chapter the implementation of boundary conditions at each vessel in- and outlet comprises the approximation of boundary characteristics together with the application of compatibility relations. Non-reflecting or open boundary conditions are implemented by using inward differences for discretisation of equation (4.14) and (4.15). By this method the outgoing characteristics do not notice the boundary at all, i.e. they leave the domain into an infinitely long tube with constant impedance. We note that great care needs to be taken with the boundary conditions to ensure that spurious solution modes do not lead to numerical instabilities. Therefore the upwind boundary is treated by upwind biased method, because the general non conservative second-order upwind scheme produces instability for explicit time differencing [136].

$$
\begin{aligned}
\left(w^{-}\right)_{0}^{n+1}= & \left(w^{-}\right)_{0}^{n}+\left(w^{-}\right)_{1}^{n}-\left(w^{-}\right)_{1}^{n-1}- \\
& \lambda^{-} \frac{d t}{d x}\left[\left(w^{-}\right)_{1}^{n}-\left(w^{-}\right)_{0}^{n}-\left(w^{-}\right)_{2}^{n-1}+\left(w^{-}\right)_{1}^{n-1}\right] \\
\left(w^{+}\right)_{I}^{n+1}= & \left(w^{+}\right)_{I}^{n}+\left(w^{+}\right)_{I-1}^{n}-\left(w^{+}\right)_{I-1}^{n-1}- \\
& \lambda^{+} \frac{d t}{d x}\left[\left(w^{+}\right)_{I}^{n}-\left(w^{+}\right)_{I-1}^{n}-\left(w^{+}\right)_{I-1}^{n-1}+\left(w^{+}\right)_{I-2}^{n-1}\right] .
\end{aligned}
$$

THE flow variables at the boundaries and the characteristics that enter the domain are obtained by the application of a specific boundary condition (in-/outflow or interface condition) and the compatibility relations in equation (4.13). Non-reflecting boundary conditions therefore simply assume that $\left(w^{-}\right)_{i=I}^{n}=0$, i.e. no left travelling characteristic $w^{-}$enters the right end of the tube. Other boundary conditions are easily implemented for different applications. 


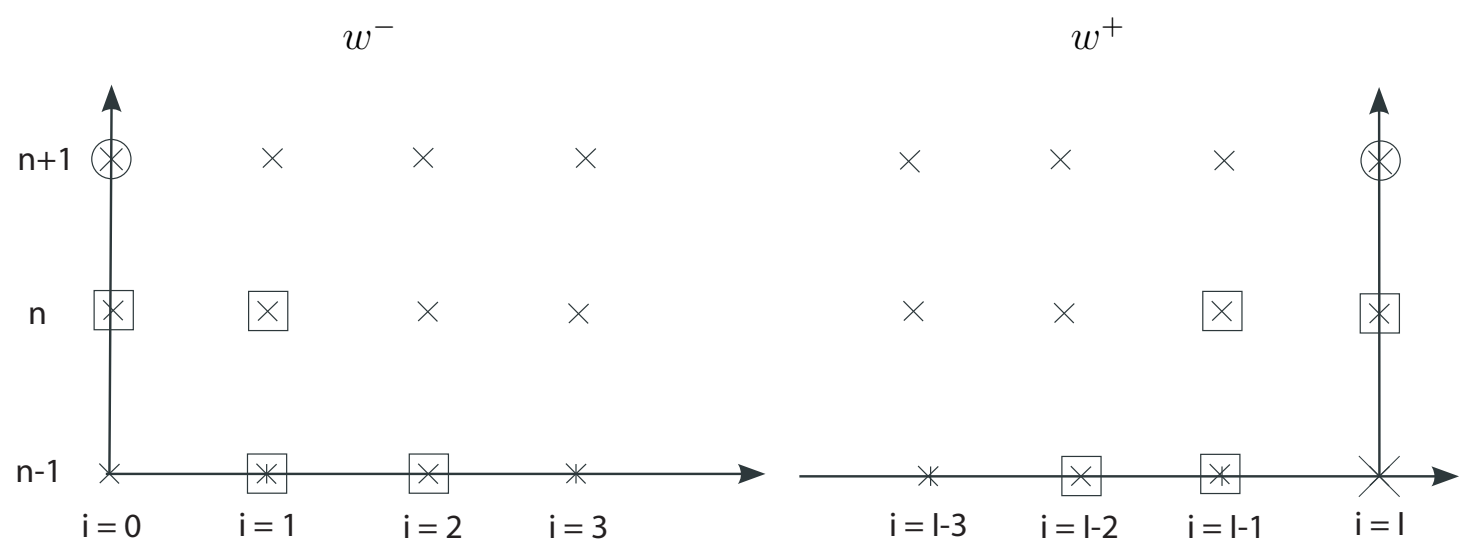

Figure 5.3: Stencils for the for the approximation of boundary characteristics. Desired values at $n+1$ are illustrated as $\circledast$, required values for the computation are illustrated as $\otimes$.

\subsubsection{Inflow condition}

AT the inlet boundary $x=0$ the variation of $A$ and $u$ is known by equation (4.18) or (4.19), so that together with the compatibility relations in equation (4.13) the entrance characteristic $w^{+}$is easily determined. However the non-linear system requires subsequent Newton iteration for solution, because the boundary condition is non-linear.

\subsubsection{Bifurcations}

ON the basis of above approximation the characteristics which enter the arterial bifurcation, $w_{p}^{+}, w_{d 1}^{-}$and $w_{d 2}^{-}$are known quantities. The reflected and transmitted characteristics in each segment are obtained by the solution of the interface conditions in equation (4.20) - (4.22). Starting point for the solution to this non-linear boundary value problem is given by analytic solution to the linearised conditions. Subsequent Newton iteration gives the final results for $A$ and $u$ in parent and daughter vessels, which through equation (4.8) and (3.43) directly lead to the required characteristics in participating segments.

\subsubsection{Outflow condition}

THE discretisation of the windkessel outflow boundary condition is taken into account by solution to equation (4.23). As before the boundary characteristics which leave the domain are known quantities, so that compatible characteristics which enter the domain are found by discretisation of equation (4.23) and iterative solution for $A$ and $u$. 


\begin{tabular}{|c|c|c|c|}
\hline \multicolumn{4}{|c|}{ read simulation parameters and initialise computational grid } \\
\hline \multicolumn{4}{|l|}{ for $n(1) N$} \\
\hline & \multicolumn{3}{|c|}{ while $\Delta \mathrm{u}<10^{-6}$} \\
\hline & & \multicolumn{2}{|c|}{$\begin{array}{l}\text { Solve inflow condition eqn. (4.18) or (4.19) } \\
\text { compatibility conditions eqn. (4.13) }\end{array}$} \\
\hline & \multicolumn{3}{|l|}{ for $m(1) M$} \\
\hline & & \multicolumn{2}{|l|}{ for $i(1) ।$} \\
\hline & & & predictor step eqn. (5.1) and (5.2) \\
\hline & & \multicolumn{2}{|c|}{ boundary characteristics eqn. (5.7) and (5.8) } \\
\hline & \multicolumn{3}{|l|}{ for $m(1) M$} \\
\hline & & \multicolumn{2}{|l|}{ for i (1) I } \\
\hline & & & corrector step eqn. (5.3) and (5.4) \\
\hline & & \multicolumn{2}{|c|}{ boundary characteristics eqn. (5.7) and (5.8) } \\
\hline & \multicolumn{3}{|l|}{ for $m(1) M$} \\
\hline & & \multicolumn{2}{|c|}{ while $\Delta \delta<10^{6}$} \\
\hline & & $\sqrt{v}$ & $\begin{array}{l}\text { von Karmann integral eqn. (5.5) and (5.6) } \\
\text { Curve fit to Falkner-Skan eqn. (3.94) and (3.95) }\end{array}$ \\
\hline & & \multicolumn{2}{|c|}{$\begin{array}{l}\text { displacement area eqn. (3.99) and (3.100) } \\
\text { momentum correction coefficient eqn. (3.102) } \\
\text { viscous friction term eqn. (3.103) }\end{array}$} \\
\hline & \multicolumn{3}{|l|}{ for $m(1) M$} \\
\hline & & \multicolumn{2}{|c|}{$\begin{array}{l}\text { satisfy boundary conditions: } \\
\text { boundary characteristics eqn. (5.7) and (5.8) }\end{array}$} \\
\hline & & \multicolumn{2}{|c|}{ while $\Delta p<10^{-6}$} \\
\hline & & & $\begin{array}{l}\text { interface conditions eqns. (4.20) - (4.22) } \\
\text { windkessel outflow condition eqn. (4.23) }\end{array}$ \\
\hline
\end{tabular}

$\mathrm{N}$ : number of time steps

M : number of segments in the network

I : number of grid points in a segment

Figure 5.4: Structogram for the boundary layer algorithm. 


\section{Chapter 6}

\section{Simulation Results}

$\mathrm{B}$ ASED on the arguments raised in the previous chapters, this chapter presents the simulation results of steady and unsteady flow in a model of the human vascular system as a typical haemodynamic application. The proposed boundary layer method to model viscous friction and momentum correction in the entrance region of a tube with varying cross-section was subsequently applied to a test geometry, which consists of a single elastic tube with either temporally fixed or dynamic indentations, and specific clinical situations of myocardial bridging in the LCA as described in [40]. The pressure drop characteristics, the mean fractional flow reserve and the wall shear stress are computed and results are compared to those available in the literature. The simulations were carried out in stenosis of different degree and extent. The Womersley and Strouhal number were around 4 and below 0.15 respectively, the Reynolds number in the stenosis varied from 850 to 1500 . The wave velocity in those situations may take values as low as $c=5 \mathrm{~m} / \mathrm{s}$ in uniform vessels, rising to values around $c=30 \mathrm{~m} / \mathrm{s}$ in constricted vessels. Physiological peak flow velocities, $\mathrm{V}$, are much smaller, generally around $0.5-1 \mathrm{~m} / \mathrm{s}$, but they can reach $2-3 \mathrm{~m} / \mathrm{s}$ in parts of severe deformation.

THE spatial resolution of the grid was adjusted to represent the curvature of deformation, where 4 gird points per mm was reasonable in any of the cases, the time resolution was chosen accordingly and ranged between $5 \mu \mathrm{s}$ and $10 \mu \mathrm{s}$. These resolutions are sufficient to avoid numerical instabilities at severe deformations. The computational time required, for the simulation of one pulsatile cycle in a network of coronary arteries, was about $22 \mathrm{~min}$. on an Apple Power-Mac G5, 2 GHz. However simulations of the main 175 arteries require considerably more computational time (about 2 to 3 hours per pulsatile cycle). We note that at least three pulsatile cycles are required to obtain steady state conditions.

GENERALly we found that under application of a uniform flow profile at the entrance the core velocity in the vessel increases gradually downstream, indicating the development of a parabolic flow profile. For a Reynolds number of 800 the distances over which the core velocity stabilised in the tube were very much shorter than at 
2000; fully in line with the dependence of the entrance length. However fully developed flow was not present in any of the cases.

IN the following we address the flow limiting effect by the assessment of the pressure derived fractional flow reserve in different stenotic environments (test geometry and LCA). Further, we will discuss the pressure drop $\Delta p$, the flow separation, the wall shear stress and the influence of external wall deformation. In some of the graphs we have shown the interval of possible solutions that may occur during the pulsatile cycle, i.e. the upper and lower envelope denoted by $\operatorname{Max}_{e n v}$ (solid red line) and $\operatorname{Min}_{\text {env }}$ (solid blue line) respectively.

\subsection{Modelling: test geometry}

WE have chosen the test geometry of the tube according to the size of the left main coronary artery with an internal diameter of $5 \mathrm{~mm}$. To observe separation and reattachment we have adapted the length to the maximum extent of the recirculation zone, which was about $300 \mathrm{~mm}$ at $80 \%$ diameter deformation. At $20 \mathrm{~mm}$ downstream of the entrance, the diameter was abruptly decreased $\left(t_{l}=4 \mathrm{~mm}\right)$ by $0-85 \%$, with an extent of $30 \mathrm{~mm}$ (single stenosis), another constriction with the same length follows $30 \mathrm{~mm}$ further downstream (double stenosis). In addition the extent of the single stenosis (short) was varied by a factor of two (medium) and three (long), in the double stenosis we varied the separation distance between one and three dimensions of the stenosis.

THE region around the diameter transition in the test geometries is shown in detail in figure 6.1. The area perturbation $A^{\prime}$, shown on the top of each column, indicates that the arterial deformation caused by pulsatile pressure is much smaller in regions where the tube is squeezed $\left(A^{\prime} / A=1-2 \%\right)$, while in circular segments $A^{\prime} / A=8-9 \%$. Due to incompressibility and mass conservation a sudden jump to a high velocity is seen at the narrowing. It appears that the stenosis influences the mean velocity only over a short distance upstream, while in the case of flow separation the core velocity slowly decays until reattachment, then it starts growing again until the flow is fully developed. The boundary layer separation in the post-stenotic region indicates an emerging top-hat velocity profile from the stenosis, with sideway counter-current flows. The decay in core flow velocity indicates shear widening of the top-hat until reattachment. Further downstream the core velocity is almost constant. It was found that the distance over which the outlet effect occurs is smaller for short stenosis with small deformation and small Reynolds number. 

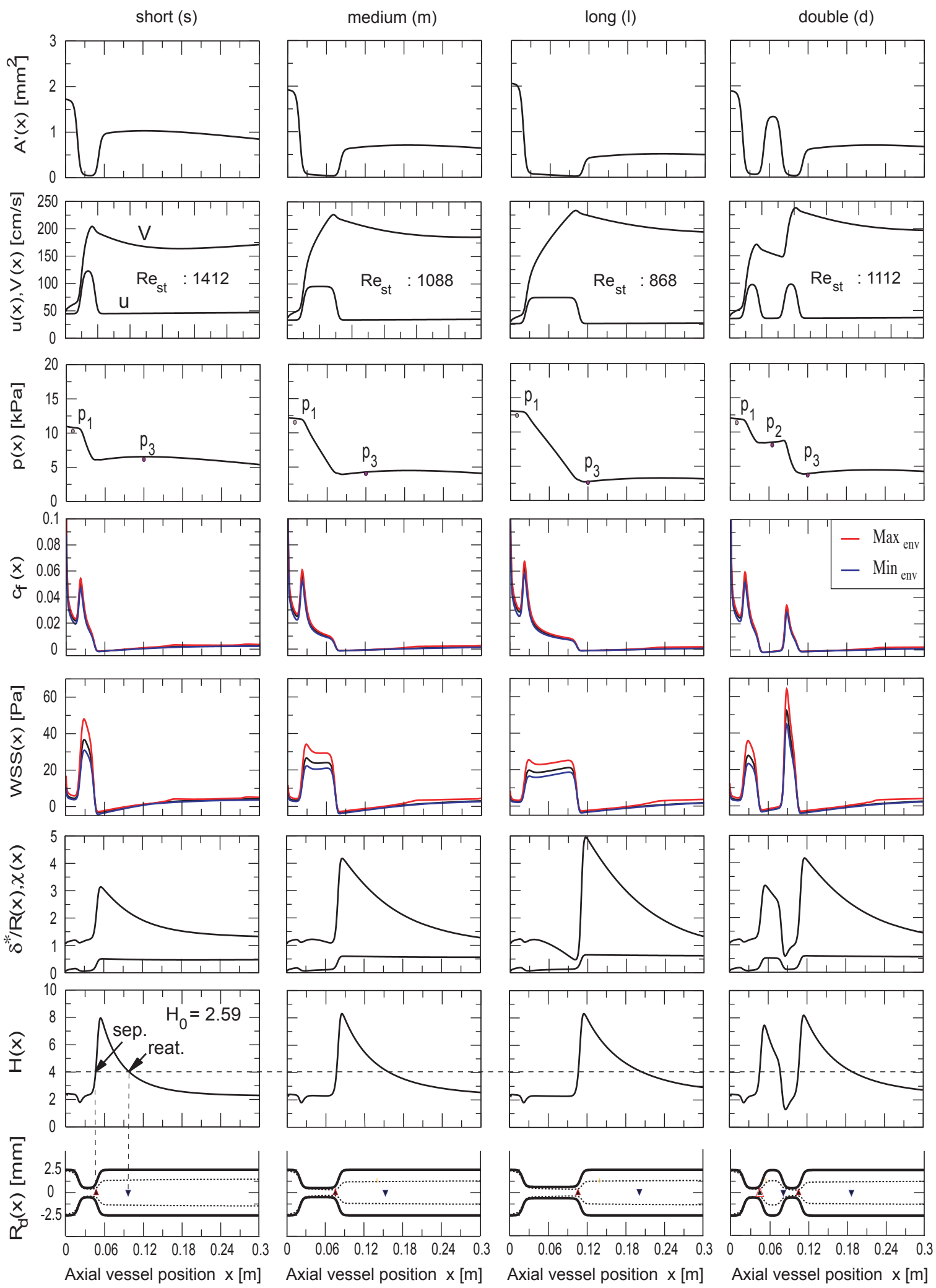

Figure 6.1: The area perturbation, flow velocity, pressure, friction coefficient, the wall shear stress, momentum correction factor, the normalised boundary layer thickness and the shape factor are plotted as a function of position in a vessel with fixed deformation of $\zeta_{0}=0.2$. Three stenoses of different extent $(\mathbf{s}),(\mathbf{m})$ and (l) and a series stenosis (d) are compared. To indicate the flow characteristics in the stenosis we have used the Reynolds number $R e_{s t}$ inside the deformation. 
(a)
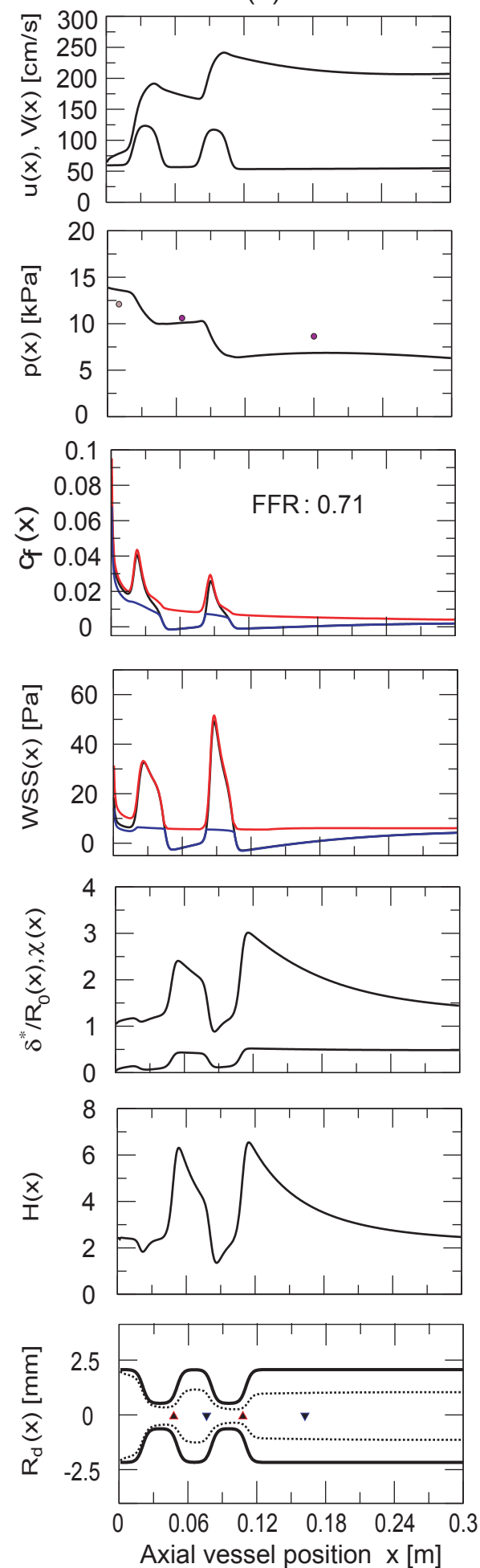

(b)
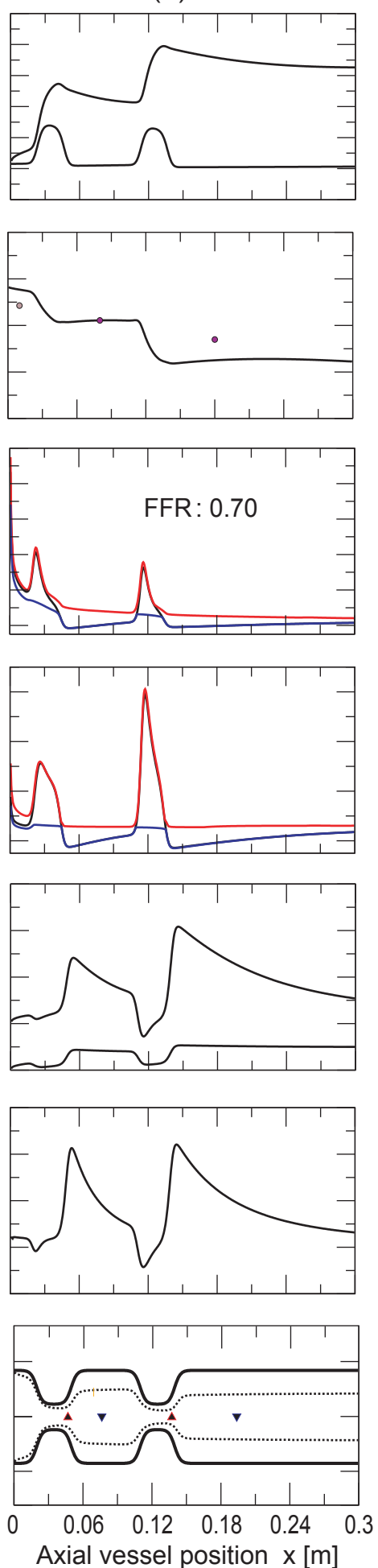

(c)
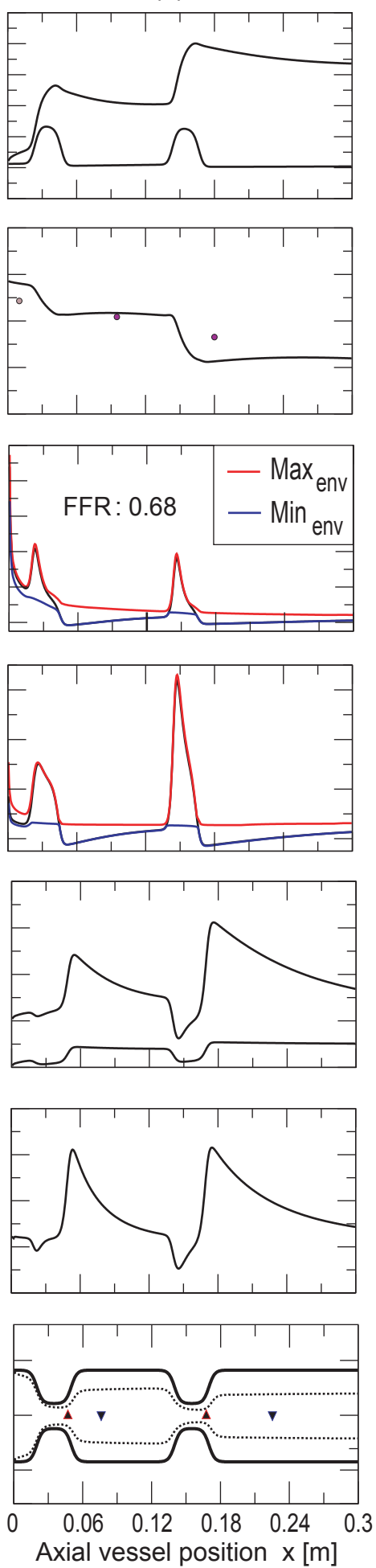

Figure 6.2: In columns (a), (b) and (c) we compare a series of two dynamic stenoses, which are separated by one, two and three stenosis dimensions respectively. From the top we illustrate the flow velocity, the pressure, the friction coefficient, the wall shear stress, the momentum correction coefficient and normalised boundary layer thickness, the shape factor and the actual geometry of the vessel. The boundary layer is illustrated as dashed lines. The FFR decreases with increasing distance between the stenoses. 
THE core velocity influences the frictional losses and the extent of the separation zone in series stenoses, so that series stenoses cannot be represented by two similar building blocks. This becomes more evident through the fact that the entrance flow profile in the second stenosis changes, if the distance of the two stenoses is varied (see figure $6.2(\mathbf{a}-\mathbf{c})$ ). In developing flow conditions the downstream deformation is generally dominant in wall shear stress and frictional forces as well as in the extent of the post-stenotic separation zone. Further, it is likely that a downstream stenosis with equal deformation collapses significantly earlier, because the core flow velocity is increased, so that the transition from subcritical flow $(S<1)$ to supercritical flow $(S>1)$ happens earlier. The following sections will discuss the major haemodynamic conditions in myocardial bridges with reference to figure 6.1 and 6.2 in further detail.

\subsubsection{Pressure drop and flow limitation}

GEOMETRIC influences on the pressure loss across series of stenoses have been studied in [74]. The authors found that the pressure drop across severe stenoses is little affected by the eccentricity of the stenosis, dominantly affected by the severity and length of the stenosis, and affected by the Reynolds number only at low Reynolds number. Further [65] found that the eccentricity of mild stenoses increases the likelihood of collapse of stenotic arteries, because the buckling pressure is reduced.

As in $[27,34]$ we found that the pressure proximal to the deformation is increased if the degree of deformation or the length of deformation is increased (see figure 6.1). Compared to the mean reference pressure (no deformation) of $9.29 \mathrm{kPa}$, the mean pressure at the inlet for a deformation of $\zeta_{0}=0.2$ was $10.28 \mathrm{kPa}, 11.53 \mathrm{kPa}$ and $12.41 \mathrm{kPa}$ in the cases (s), (m) and (l) respectively. Under these conditions the mean flow in the reference artery was $10.36 \mathrm{~cm}^{3} / \mathrm{s}$. For fixed deformation of $\zeta_{0}=0.2$ we found $8.43 \mathrm{~cm}^{3} / \mathrm{s}, 6.38 \mathrm{~cm}^{3} / \mathrm{s}, 4.94 \mathrm{~cm}^{3} / \mathrm{s}$ and $6.66 \mathrm{~cm}^{3} / \mathrm{s}$ in the short, medium, long and double stenosis respectively. The pressure drop for the medium sized stenosis was at $7.52 \mathrm{kPa}$ just below that of the double environment with $7.78 \mathrm{kPa}$, the pressure drop across the short stenosis was $4.15 \mathrm{kPa}$ and across the long stenosis $9.82 \mathrm{kPa}$. In double stenoses the pressure drop across the proximal stenosis was $3.28 \mathrm{kPa}$, which is markedly less than $4.4 \mathrm{kPa}$ at the distal stenosis. Complementary the values for the dynamic case are generally less pronounced, they can be found in table 6.1.

However, the translesional pressure drop in a series of two stenoses in the entrance region of a tube shows further remarkable effects. The simulations indicate that in general the pressure drop cannot be obtained by a summation of pressure drops for single stenosis, since the proximal and distal stenosis influence each other unless the spacing between them exceeds some critical distance, which depends on the Reynolds number and deformation. Therefore several consecutive stenoses along the same epicardial artery require separate determination of stenosis severity. We found that when two stenoses are close together, the pressure drop is approximately equal to that of a 
Table 6.1: Mean flow quantities, pressure drop and fractional flow reserve for test geometry shown in figure 6.1

\begin{tabular}{|c|c|c|c|c|c|c|c|c|c|c|}
\hline mea & unit & \multicolumn{4}{|c|}{ fixed } & \multicolumn{4}{|c|}{ dynamic } & ref \\
\hline & & $(\mathrm{s})$ & $(\mathrm{m})$ & (1) & $(\mathrm{d})$ & $(\mathrm{s})$ & $(\mathrm{m})$ & $\overline{(1)}$ & $\overline{(d)}$ & \\
\hline $\bar{p}_{1}$ & $k P a$ & 10.42 & 11.63 & 12.48 & 11.46 & 9.55 & 9.84 & 10.07 & 9.80 & 9.29 \\
\hline$\triangle p_{1-3}$ & $k P a$ & 4.15 & 7.52 & 9.82 & 7.78 & 1.89 & 2.74 & 3.47 & 2.87 & 1.23 \\
\hline$F F R_{p}$ & $\%$ & 60 & 35 & 21 & 32 & 80 & 72 & 65 & 70 & 86 \\
\hline $\bar{q}_{1}$ & $\mathrm{~cm}^{3} / \mathrm{s}$ & 8.43 & 6.38 & 4.94 & 6.66 & 9.90 & 9.40 & 9.01 & 9.46 & 10.36 \\
\hline $\bar{u}_{1}$ & $\mathrm{~m} / \mathrm{s}$ & 0.43 & 0.33 & 0.25 & 0.34 & 0.50 & 0.48 & 0.46 & 0.48 & 0.53 \\
\hline
\end{tabular}

stenosis with twice the length of a single stenosis (see table 6.1 and figure $6.1(\mathbf{m})$ and (d)). This can be explained by the fact that the friction coefficient in the recirculation zone between the two stenoses is small and consequently the pressure loss over that region is small. However, when the stenoses are separated by more than the length of the stenosis the flow is hardly affected by the second stenosis, because the core flow velocity and likewise the frictional force are increased (see figure $6.2(\mathbf{a}-\mathbf{c})$ ), even though the entrance flow conditions are preserved. Coexistent is the increased reduction in shape factor and momentum correction coefficient, suggesting the non-linear influence in that region. The pressure drop over the downstream deformation is generally pronounced, however the distance between the deformations further increases the pressure drop and consequently the mean FFR is reduced. The values for the cases (a) to (c) are $\triangle p_{a}=3.45 \mathrm{kPa}, \triangle p_{b}=3.64 \mathrm{kPa}$ and $\triangle p_{c}=3.89 \mathrm{kPa}$ for the pressure drop over the downstream deformation and $F F R_{a}=71 \%, F F R_{b}=70 \%$ and $F F R_{c}=68 \%$ for the mean fractional flow reserve across the bridge. We note that these findings are based on the entrance type of flow, where the core flow velocity gradually changes and may not appear in fully developed flow, where the core flow velocity is a constant and identical to the maximum of Hagen-Poiseuille flow velocity.

\subsubsection{Separation and reattachment}

THE core velocity in a uniform tube generally increases downstream of the entrance, however in presence of a stenosis the boundary layer thickness decreases at the inlet and rapidly increases in the diverging part of the constriction (see figure 6.1). Separation occurs under the development of a top hat profile with sideway counter-current flow. At the separation point $(\boldsymbol{\Lambda})$ the boundary layer thickness increases and a sudden jump in $\chi$ is evident, because the integral of the actual velocity $v_{x}^{2}$ over the area of the recirculation is close to zero and in contrast to the mean flow velocity the core velocity is increased. Therefore the non-linear term is pronounced in the separation region, while it is close to unity in converging regions. We note that the momentum correction becomes markedly smaller than before the upstream stenosis. This indicates that the 
entrance profile into the second stenosis is almost flat, but has increased core velocity, while the counter current flow at the wall has disappeared. The reattachment of the boundary layer $(\boldsymbol{\nabla})$ further downstream is caused by momentum diffusion from the inviscid core into the the recirculation zone and the friction between the shear layers. The widening of the central core reduces the flow velocity which causes the pressure to recover. If the core flow is close to the vessel wall it cannot draw fluid through the wall and the core flow itself is attracted towards the wall, attaching itself to the wall. A similar phenomenon occurs with boundary layers. In the theory of laminar boundary layers the condition for separation and reattachment is, as the shape factor $H>4$ and as it drops below the critical value of 4 respectively. Due to the increased momentum correction in that region the pressure in the non-linear case recovers more rapidly than in linearised computations, which causes earlier reattachment and consequently slightly smaller recirculation zones. The extent of the recirculation zone is primarily dependent on vessel deformation and Reynolds number. The separation point moves upstream, while the reattachment point moves downstream if the Reynolds number or deformation increases. However, we found that the extent also correlates with the length of the constriction. Compared to the short deformation in figure 6.1 ( $\mathbf{s}$ ) the tail of the shape factor curve drops below the critical value of 4 (condition for reattachment) by a factor of about two and three later for the medium ( $\mathbf{m}$ ) and long (1) constriction respectively. In other words vessels with the same degree of stenosis, but with the stenosis having different curvatures and lengths, have recirculation regions that differ markedly in their extent. At deformations of $85 \%$ the recirculation zones had an extent of about 20 tube diameters in length. A particularity of series stenoses is that the extent of the recirculation zone in the interconnecting segment is reduced. This is due to early reattachment caused by fluid acceleration in the converging part of the distal stenosis. But nonetheless the core flow velocity and though the Reynolds number in the proximal stenosis is generally smaller than in the distal stenosis.

\subsubsection{Wall shear stress and friction coefficient}

ShEAR stress for a Newtonian fluid is conventionally estimated from gradients of measured or simulated velocity components, and the fluid viscosity. This process involves some approximations which may lead to underestimation on the order of $10 \%-45 \%$, when the lumen is not circular [137]. This large error is claimed to be due to nonlinear velocity profile at the wall and ignorance of the radial derivatives.

For steady flows the location of maximum wall shear is always upstream the neck of the stenosis (see figure 6.1), and moves upstream as the Reynolds number increases. In series stenoses the wall shear stress is significantly increased in the distal stenosis, while the friction coefficient is smaller there (see column (d)). Generally they have their maximum at the entrance of the stenosis and reduce towards the end of the stenosed section. Eventually they become negative after separation of the boundary 
layer. The increased boundary layer thickness in the downstream stenosis suggests lower retarding forces, however, the core flow velocity is increased there so that pressure losses are dominant there. Consequently the second stenosis can be seen as the more vulnerable, in wall shear stress, frictional forces and consequently in flow limitation. Likewise the mean flow velocity in a pressure driven vessel is dependent on the total after-load, the maximum values of wall shear stress are found in short constrictions (see column (s)), because the after-load is smaller and fluid velocity is increased compared to long constrictions (column (l)). Although the wall shear is increased in short constrictions, we observe that the peak of the viscous friction coefficient increases if the length of the constriction is increased. In flow driven vessels however the peak values are independent of the extent, because the flow velocity is equal in all cases (not shown here).

THE wall shear stress is large in the entrance region of the deformation, fades towards the end and is negative in the separation region, so that the development of atherosclerosis is more likely in segments proximal to the deformation. Compared to wall shear stresses in non-diseased vessels $\left(5-10 \mathrm{~N} / \mathrm{m}^{2}\right)$ vulnerable regions are endothelial cells in the throat of a strong deformation. They may experience wall shear stresses in excess of $60 \mathrm{~N} / \mathrm{m}^{2}$. In series stenoses the wall shear stress is largest in the downstream stenosis because the core flow velocity is increased there. Furthermore we note that the wall shear stresses are no longer likely to be distributed evenly around the circumference of the vessel and may be particularly focused on the most vulnerable shoulder regions, marking the transition from normal to diseased artery wall. Further improvements for the prediction of the wall shear stress may be obtained by the introduction of a shear dependent model to predict the local blood viscosity.

\subsubsection{Unsteady solutions}

DESPITE the assumption of strong coupling between the boundary layers and the core flow and the assumption of quasi-stationary evolution of the boundary layers, the time dependent motion of the wall under external deformation reproduces some remarkable characteristics of myocardial bridges. In figure 6.3 we have shown the effect of temporal deformation onto the pressure and flow in the segments $\Omega_{s 1-s 5}$ of a series of two myocardial bridges (see figure 3.1). The time dependence of the two separation zones, one between the two deformations and the other distal to the second deformation shows that separation occurs for deformations greater than about $40 \%$. The separation cycle is present in the time interval of $0.1 s$ to $0.4 s$. The maximum deformation during the cycle was $75 \%$ of $R_{0}$, which was reached at $0.3 \mathrm{~s}$. It is seen that during deformation the separation point moves slightly upstream (lower part of the cycle), while the reattachment point (upper part of the cycle) of the boundary layer moves farther downstream. The upstream separation zone (turquoise cycle) is spread over a region of $49.94 \mathrm{~mm}$ to $80.83 \mathrm{~mm}$, while the downstream separation zone 

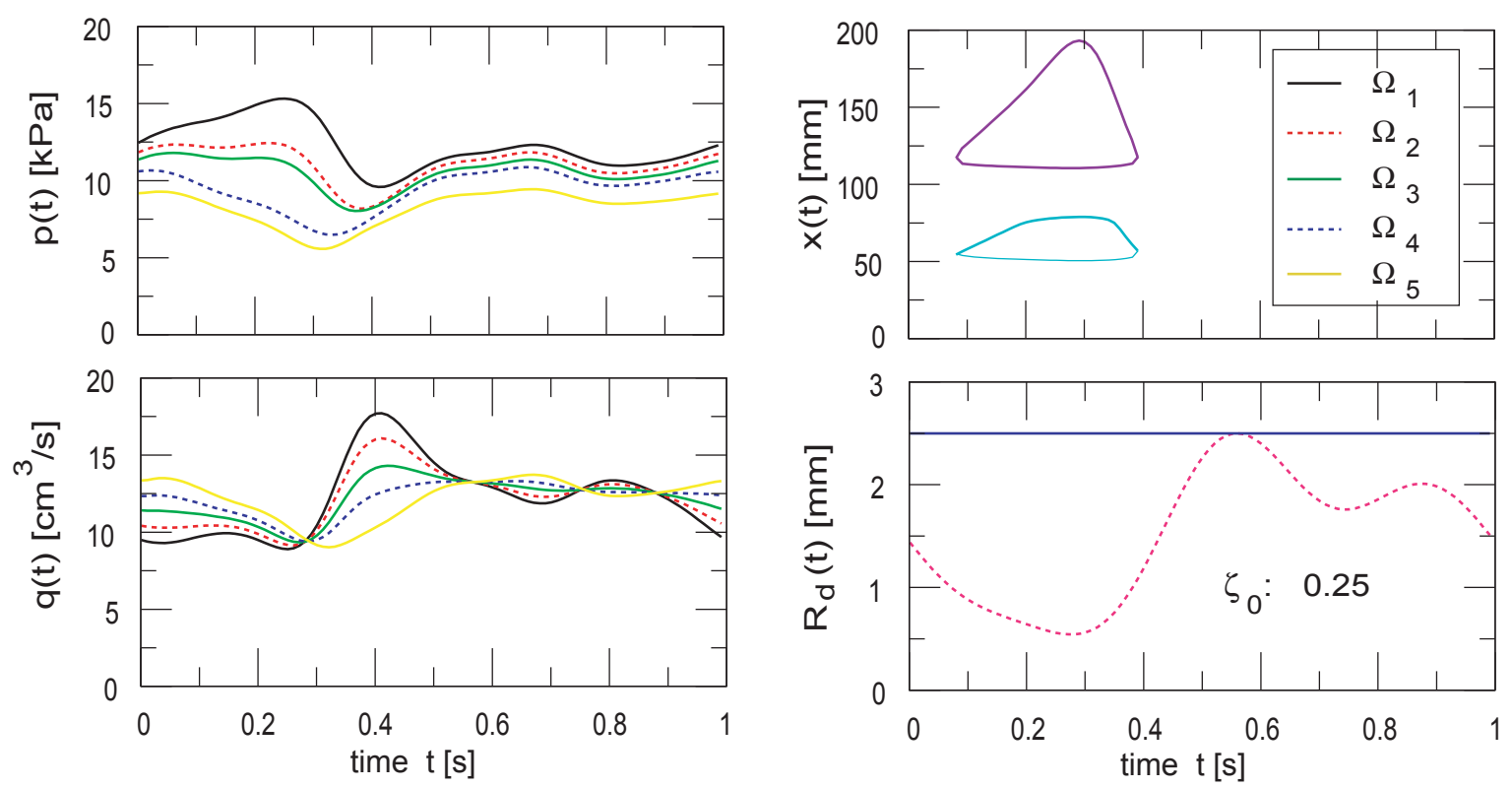

Figure 6.3: The temporal dependence of pressure and volume flow (left) and the separation cycle during deformation (right) of a series of two myocardial bridges given in figure 6.2 (a) are illustrated. The dashed lines represent flow variables in segments with deformation $\left(\Omega_{s 2}\right.$ (red) and $\Omega_{s 5}$ (blue)), while solid lines show the flow variables in segments without deformation $\left(\Omega_{s 1}\right.$ (black) proximal, $\Omega_{s 3}$ (green) in the centre and $\Omega_{s 6}$ (yellow) distal to the stenoses). Separation and reattachment during the cardiac cycle was observed for severe deformation between $0.1 s$ and $0.4 s$ (right).

(purple cycle) is from $109.98 \mathrm{~mm}$ to $193.32 \mathrm{~mm}$. The extensions of the two zones differ, because the upstream reattachment point is forced early due to accelerating fluid at the inlet of the downstream deformation and because the core velocity is generally larger farther downstream. In each case however, a top-hat velocity profile develops in systole, producing large recirculation zones distal to the stenoses, which are delayed into diastole. The back-flow is developing earlier for severe deformation and is strongly dependent on deformation phase. For phase delayed deformation, say, by half the cycle time, the flow patterns are noticeably different (not shown here). The top-hat profile is now present in diastole, the peak velocities and the viscous friction are less pronounced and the reverse flow region is smaller and mainly present in the diastole. The mean FFR was increased to 0.65 compared to 0.6 of the zero phase case. This can be explained by the circumstance that for the phase delayed deformation no appreciable pressure drop was present at the downstream stenosis. 


\subsection{Modelling: physiological basis}

FLOW in the coronary arteries is complicated by several important features. Firstly, flow in the left main coronary artery is of entrance type, because the artery is relatively short. Secondly, the Womersley number is arround 5, so that the viscous forces do not dominate inertial forces and the assumption of Hagen-Poiseuille flow is not applicable. Thirdly, most of the volume flow in the left coronary artery occurs during diastole and high pressure in the myocardium may cause reverse flow during systole. And finally, the time dependent deformation in the myocardial bridge produces secondary flows during the cardiac cycle. The simulation results are based on a coronary network topology presented in $[138,139]$. Parameters and exact course of the arteries are given in table A.1 and figure A.1 in the appendix.
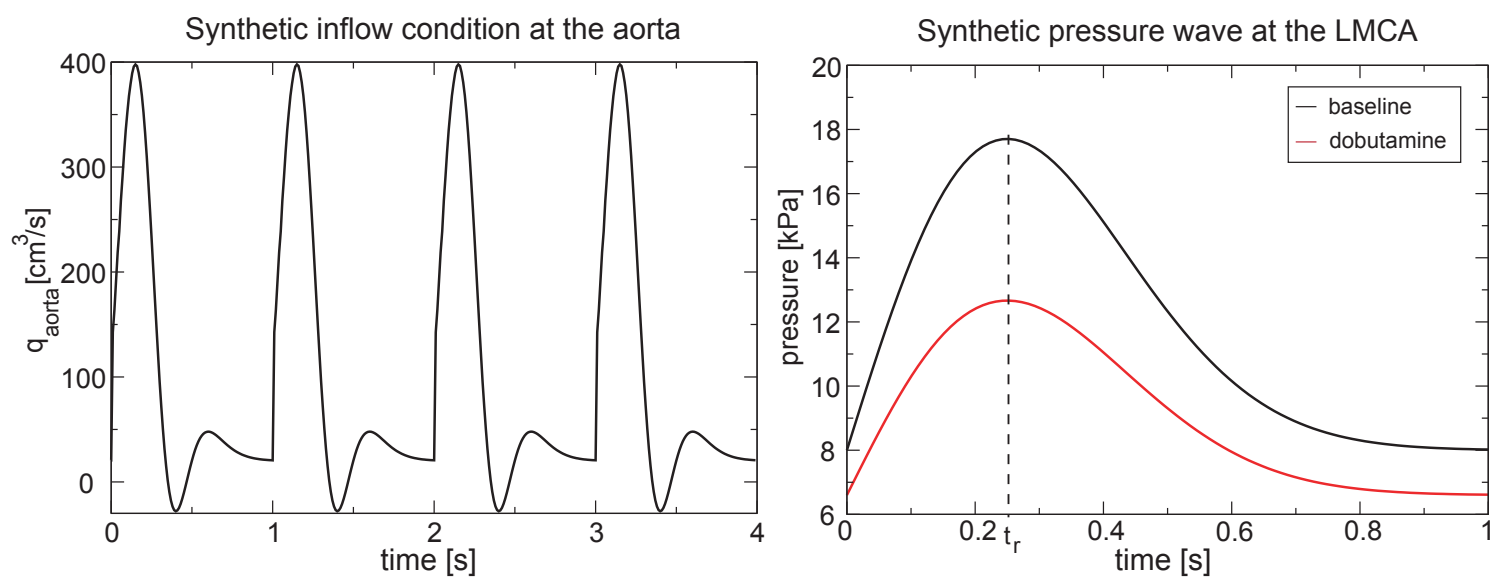

Figure 6.4: Physiological boundary conditions at the inlet are either represented by a synthetic flow wave (left) or by a pressure wave (right). The inflow condition at the aorta is modelled by equation (4.18) and the pressure condition at the inlet of the LMCA is modelled by equation (4.19). The parameters at baseline conditions (black line) and under dobutamine (red line) where chosen according to measurements in [40] and are given in the text.

THE results were obtained for a specific set of parameters [40] under resting conditions (baseline) and conditions of physical exercise, which were provocated with dobutamine. The length of the myocardial bridge for the baseline and dobutamine case were $12 \mathrm{~mm}$ and $24 \mathrm{~mm}$ respectively. The values for the deformation were $\zeta_{b}=0.54$ and $\zeta_{d}=0.32$ (see figure 6.5). We note that as the degree of deformation increases a subsequent change in deformation length is observed in most patients [40]. In principle this property can be modelled, even though the relation is patient specific. In this principle study however, we assume that the deformation length is independent of severity. To assess the dynamics, more physiological waveforms were applied, either to 
the aorta (see figure 6.4 (left)) or to the inlet of the left main coronary artery (LMCA) (see figure 6.4 (right)). The peak Reynolds and Strouhal numbers in the myocardial bridge were 815 and 1069, and 0.021 and 0.016 for the baseline and dobutamine case respectively. The Womersley number was 4.11 .

\subsubsection{Mean pressure drop}

THE mean pressure drop was calculated by subtracting the average of a distal pressure wave, which was taken approximately $3 \mathrm{~cm}$ distal to the myocardial bridge, from the average inlet pressure at the LMCA. The proximal and distal pressure waves for the baseline and while dobutamine challenge are shown in figure 6.5 (b). It is seen that the baseline pressure is less affected, while the pressure under dobutamine challenge shows a pressure notch, which may appear if the deformation is dominant during systole (see earlier discussion on that in [99]). At baseline the mean pressure at the inlet was $p_{p}=11.92 \mathrm{kPa}$ and the mean distal pressure was $p_{d}=10.67 \mathrm{kPa}$, so that the pressure drop was $\triangle p=1.25 \mathrm{kPa}$, which compares well with the value of measurements mentioned above, where $\triangle p=1.19 \mathrm{kPa}$. Under dobutamine challenge the corresponding values are $p_{p}=9.64 \mathrm{kPa}, p_{d}=8.1 \mathrm{kPa}$ and $\triangle p=1.54 \mathrm{kPa}$, which however are close to the measured values, where $\triangle p=1.85 \mathrm{kPa}$. We note that at severe deformations the flow conditions may be turbulent, so that the pressure drop is underestimated. This would explain why the model reproduces the pressure drop at moderate deformations, while it performs worse at severe deformations.

WE have further compared the translesional pressure drop across a series of two myocardial bridges resulting from Hagen-Poiseuille and boundary layer computations in three time series (see figure 6.6), one as reference, without a stenosis (time series 1), one assuming a Hagen-Poiseuille flow with a series stenosis, each of length $8 \mathrm{~mm}$ and deformation $\zeta_{0}=0.25$ (time series 2) and the same series stenosis with the boundary layer method described here (time series 3 ). The instantaneous pictures where taken at four relevant times at $0.05 s, 0.25 s, 0.5 s$ and at $0.9 s$. It is clearly seen that the systolic pressure in time series 1 is uniformly distributed and fades towards the terminals of the network. In time series 2 we notice that during systole the pressure drops in both of the stenoses, so that the LAD branch is less distributed. However the boundary layer computations in time series 3 show that the pressure drop by the assumption of fully developed flow was underestimated in the case of developing flow conditions.

\subsubsection{Pressure-flow relation}

THE pressure-flow relations for different stenotic environments in the coronary arteries are shown in figure 6.7. We have applied a stationary flow rate $q_{L M C A}$ at the 
(a)
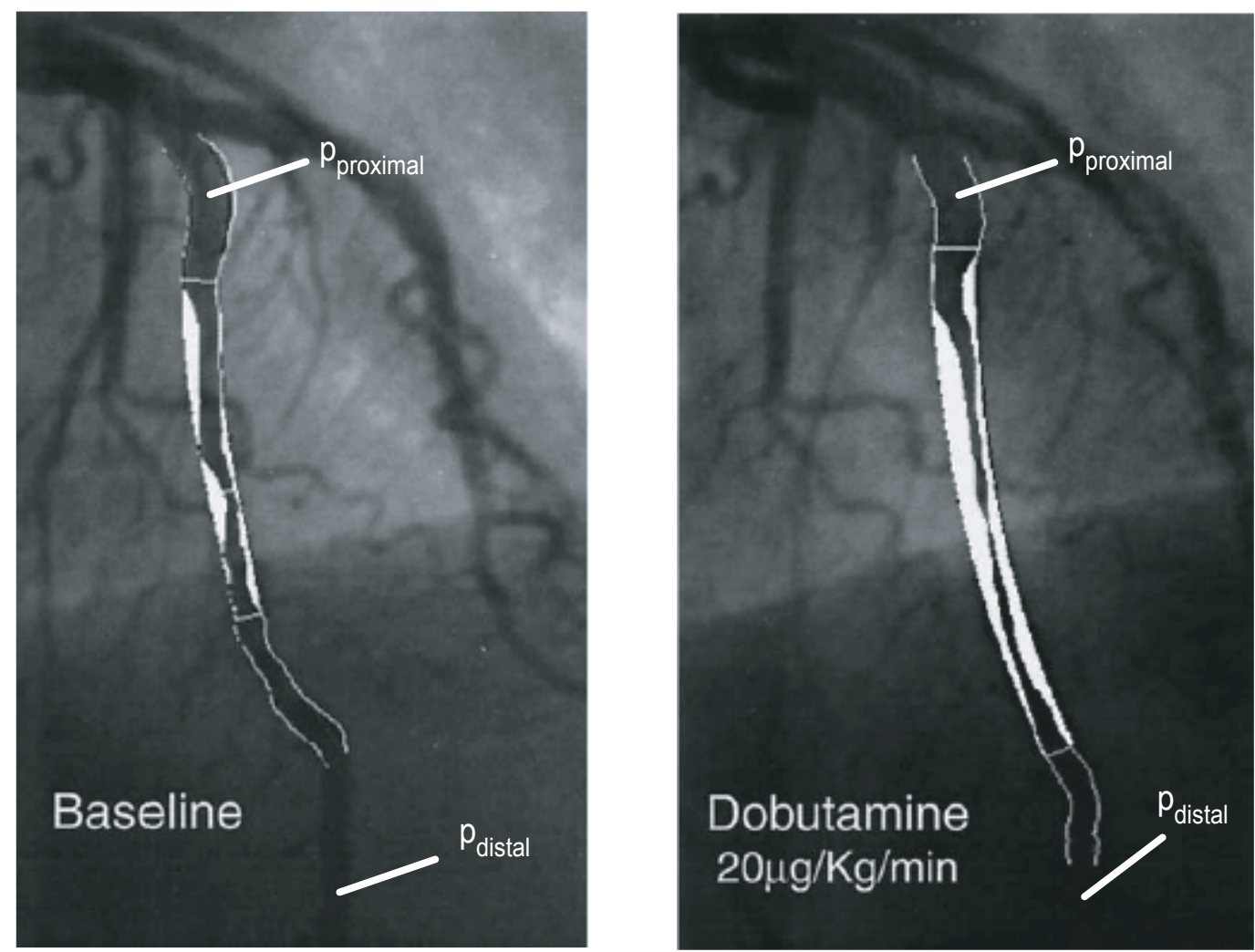

(b)

Baseline

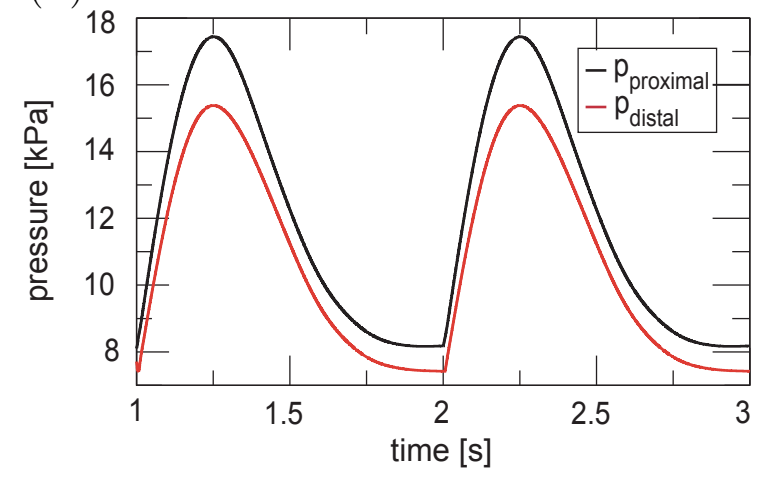

Dobutamine

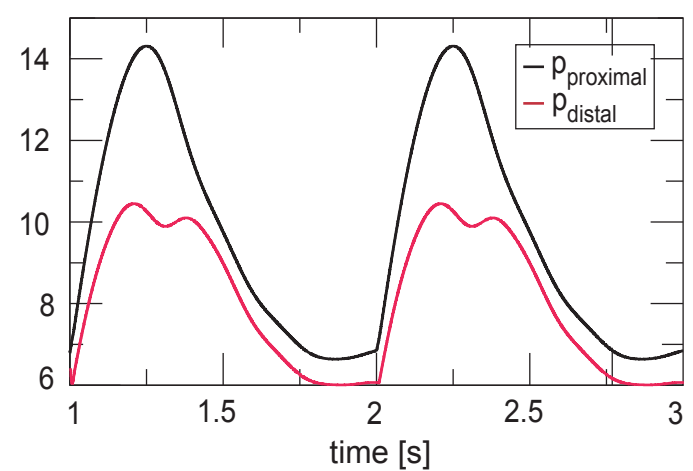

Figure 6.5: (a) shows an angiographic image of a myocardial bridge in baseline conditions (left) and while dobutamine challange (right). Dobutamine produces conditions of maximum hyperemia where the peripheral resistance is minimised. The heart rate and contraction of the heart muscle are increased. The locations for pressure measurement proximal and distal to the myocardial bridge are shown. modified from [40]. (b) shows the simulation of intracoronary pressure at baseline and during dobutamine challenge. The proximal pressure, $p_{\text {proximal }}$ was taken from the inlet of the coronary tree (LMCA), the distal pressure, $p_{\text {distal }}$ in a segment $3 \mathrm{~cm}$ distal to the myocardial bridge (see (a)). At baseline the mean FFR was 0.90, while during dobutamine challenge the mean FFR was 0.84 . The corresponding pressure drops were $\triangle p=1.25 \mathrm{kPa}$ and $\triangle p=1.54 k P a$ respectively. 


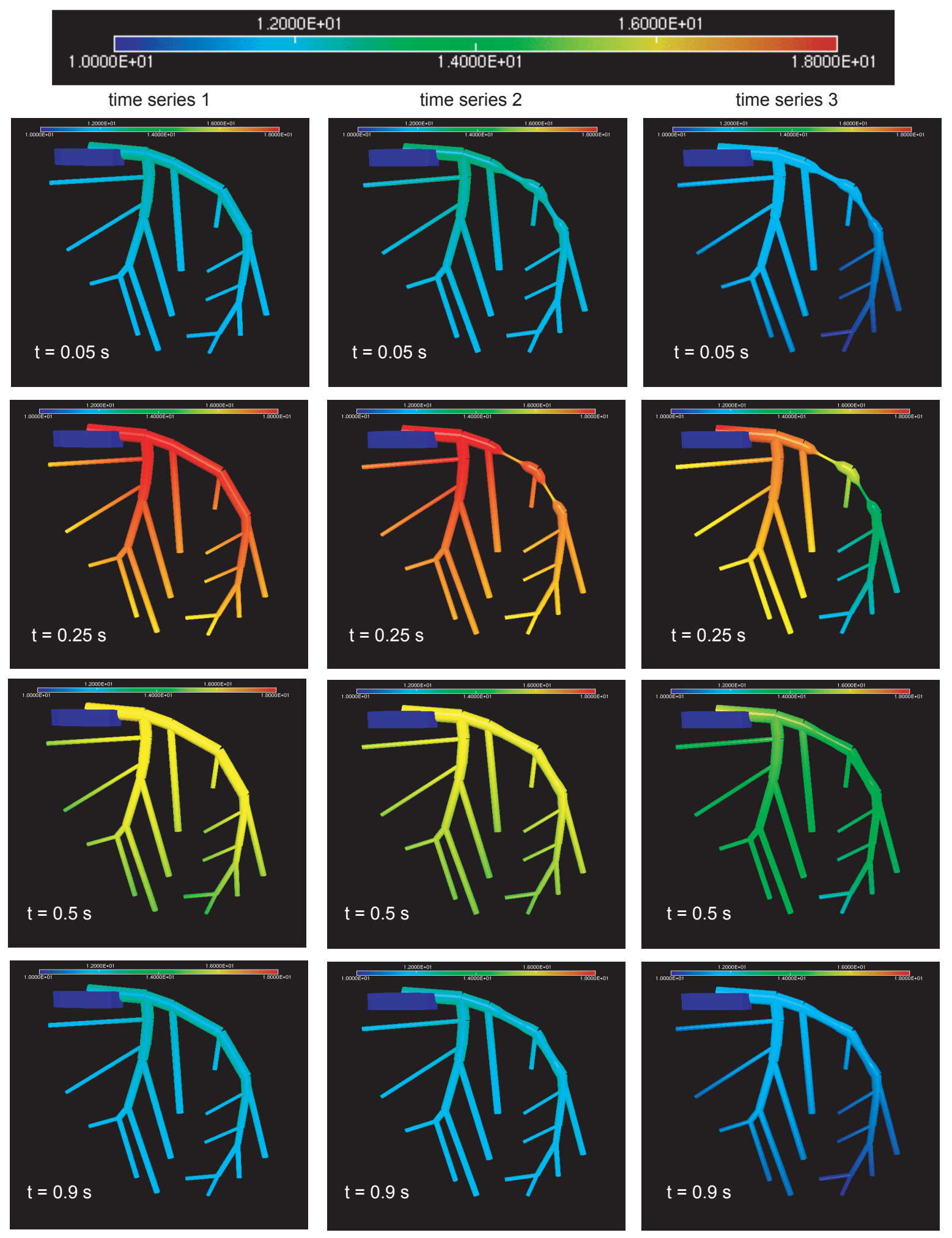

Figure 6.6: Time series of three simulations in the LCA. The pressure distribution in time series 1 is uniformly throughout the series (reference), while in time series 2 the pressure drops in a series of two myocardial bridges (Hagen-Poisuille). Developing flow conditions are illustrated in time series 3 (boundary layer theory). The pressure scale is in $k P a$. 


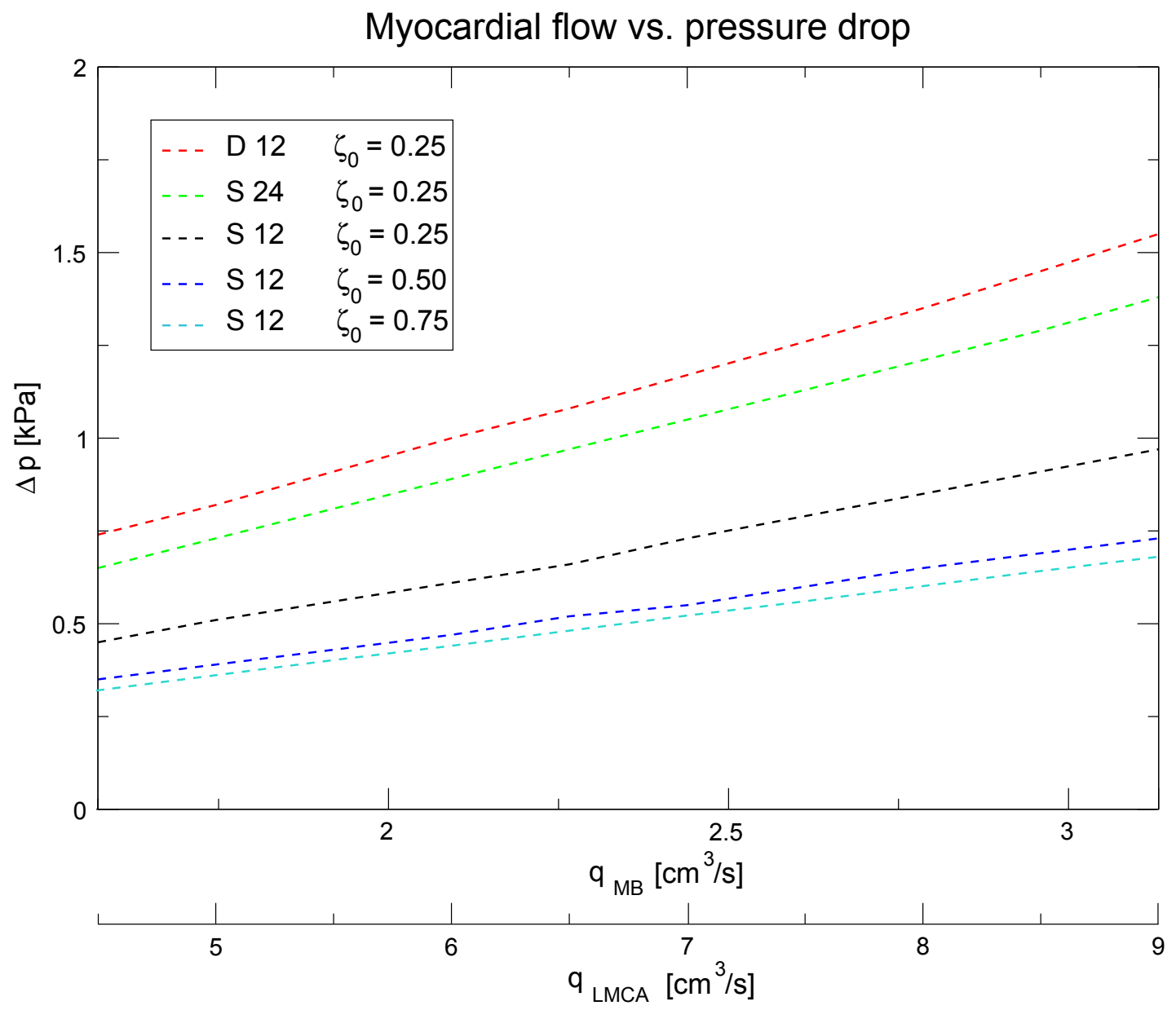

Figure 6.7: The pressure-drop flow characteristics of stenoses with different degree and extent where obtained under stationary flow conditions. We compare two single dynamic stenosis of length $12 \mathrm{~mm}$ (S12) and $24 \mathrm{~mm}$ (S24) with a double stenosis with length of $12 \mathrm{~mm}$ each (D12). Further D12 is compared for three severities $\zeta_{0}=$ $0.25,0.5$ and 0.75 . The relations are essentially linear, extrapolation to lower inflow values leads to the origin.

entrance of the LMCA in the range between $4.5 \mathrm{~cm}^{3} / \mathrm{s}$ and $9 \mathrm{~cm}^{3} / \mathrm{s}$. The resultant mean flow in the myocardial bridge, $q_{M B}$ was between $1.57 \mathrm{~cm}^{3} / \mathrm{s}$ and $3.13 \mathrm{~cm}^{3} / \mathrm{s}$. The pressure-flow relations in this range are essentially linear and extrapolate to the origin at $q_{L M C A}=0$ and $\triangle p=0$. This is consistent with the assumption of long waves in short tubes, where the pressure-drop is linearly dependent on the flow. The single deformation with length $12 \mathrm{~mm}$ is denoted by (S 12) and with length $24 \mathrm{~mm}$ by (S 24). We further show a double deformation with length $12 \mathrm{~mm}$ (D 12). In general it is seen that the pressure drop increases with severity and length of the deformation, however the difference between S 24 and D 12, which have essentially the same deformation length, result from inlet and outlet effects of the double environment. 


\subsubsection{Fractional flow reserve}

THE flow limitation caused by epicardial stenoses is generally expressed by the flow based FFR, which is the ratio of hyperaemic myocardial blood flow in the presence of a stenosis to hyperaemic flow in the absence of a stenosis, $F F R_{q}=q_{s} / q_{n}$, i.e. the flow based FFR is the fraction of hyperaemic flow that is preserved despite the presence of a stenosis in the epicardial coronary artery. However this definition is purely theoretical, because the flow without the stenosis is not known, so that for clinical purposes the ratio of hyperaemic flows with or without a single stenosis is derived from the mean distal coronary pressure, $p_{d}$, to mean proximal pressure, $p_{p}$, both recorded simultaneously under conditions of maximum hyperaemia. Neglecting correction terms the mean pressure-derived fractional flow reserve is $F F R_{p}=p_{d} / p_{p}$.

TAKING the pressure values resulting from boundary layer computations of the previous section, we obtain values for the mean pressure derived FFR of 0.90 and 0.84 for baseline conditions and under dobutamine challenge respectively. These values agree with the measurements in [40], where the values were 0.90 and 0.84 respectively.

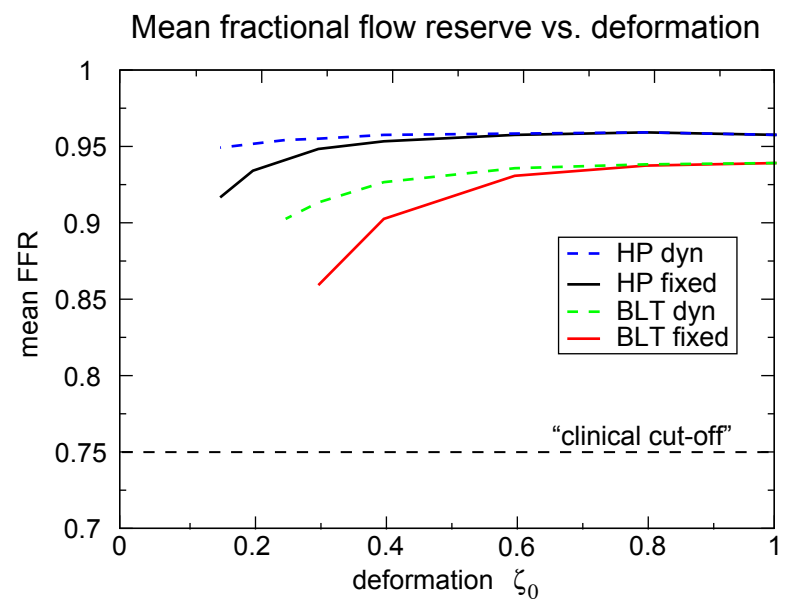

Fractional flow reserve vs. deformation length

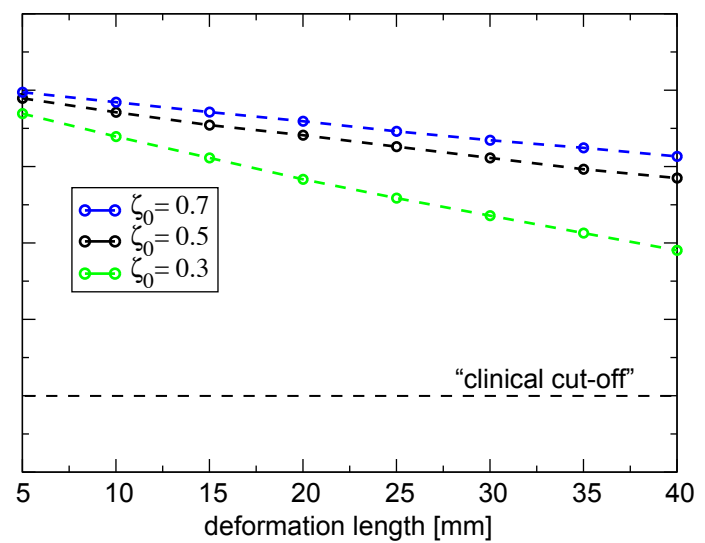

Figure 6.8: The mean pressure derived fractional flow reserve of the baseline environment shown in figure 6.5 (a) is plotted as a function of deformation (left) defined in equation (3.30) and versus the segment length (right). The computations via the boundary layer theory (BLT) are compared to Hagen-Poiseuille (HP) flow profile $(\gamma=2)$. It is seen that the mean pressure derived fractional flow reserve is overestimated under the assumption of fully developed flow. Generally the mean FFR is larger in the dynamic case (dashed lines), because the pressure recovers during the relaxation phase. The segment length of three dynamic lesions at a deformation of $\zeta_{0}=0.7,0.5$ and 0.3 was varied in a physiological range between $5 \mathrm{~mm}$ and $40 \mathrm{~mm}$. The relation is essentially linear, which indicates that for the observed deformations the losses are mainly viscous and that the non-linear term plays a minor role. 
In figure 6.8 we have shown the coronary pressure-derived fractional flow reserve for the baseline case as a measure of coronary stenosis severity (left) and in dependence on deformation length (right). In both plots we have used baseline inflow conditions with either fixed (solid lines) and dynamic walls (dashed lines). Initially we found that in developing flow conditions the FFR is overestimated by the assumption of Hagen-Poiseuille flow (HP), and, as expected, that the mean FFR in dynamic lesions is much larger than in fixed stenotic environment, because the distal pressure recovers during relaxation phase. The graph at the right shows that the mean FFR depends essentially linearly on deformation length for different severities, $\zeta_{0}=0.7,0.5,0.3$, suggesting that the losses are mainly viscous and that the non-linear term plays a minor role for observed deformations.

THE values indicate that the FFR depends on the degree and length of the stenosis. As mentioned earlier the losses in fixed environments are more pronounced. Further, we point out that series stenoses separated by more than the length of the stenosis drop below the cut-off value of 0.75 markedly earlier than single stenosis with the same degree and extent. In the case of two consecutive stenoses, however, the fluid dynamic interaction between the stenoses alters their relative severity and complicates determination of the FFR for each stenosis separately from a simple pressure ratio as in a single stenosis. Consequently the FFR determined for single stenosis is unreliable in predicting to what extent a proximal lesion will influence myocardial flow after complete relief of the distal stenosis and vice versa.

\subsubsection{Influence of wall velocity}

IN contrast to fixed stenoses, the velocity of the wall $v_{w} \neq 0$ in a dynamic environment, i.e. positive during compression and negative while relaxation. At the bottom of figure 6.9 we compare the thickness of the boundary layer during inward (left) and outward motion (right) of the wall to fixed deformations (dashed line). The situation depicts two different states where the influence is close to its maximum. Compared to fixed deformations $\left(v_{w}=0\right)$ the boundary layer thickness in systole is increased in the entrance region of the deformation, while it is decreased in the outlet region, and the situation is vice versa in diastole. In fact this is due to an additional pressure gradient, which causes fluid acceleration or deceleration depending on axial position in the constriction and whether the wall moves inwards or outwards. For example during inwards motion of the wall the fluid is decelerated at the entrance and accelerated at the outlet of the deformation, while the situation is opposite if the vessel wall moves outwards. Due to the symmetry of the indentation there is no additional acceleration or deceleration in the centre of the deformation. The influence of the wall velocity onto the boundary layer properties is more pronounced if $\frac{v_{w}}{V}$ is reasonably large, i.e. if the deformation that the axial flow experiences during passage of the constriction is comparable to the radius of the tube. 

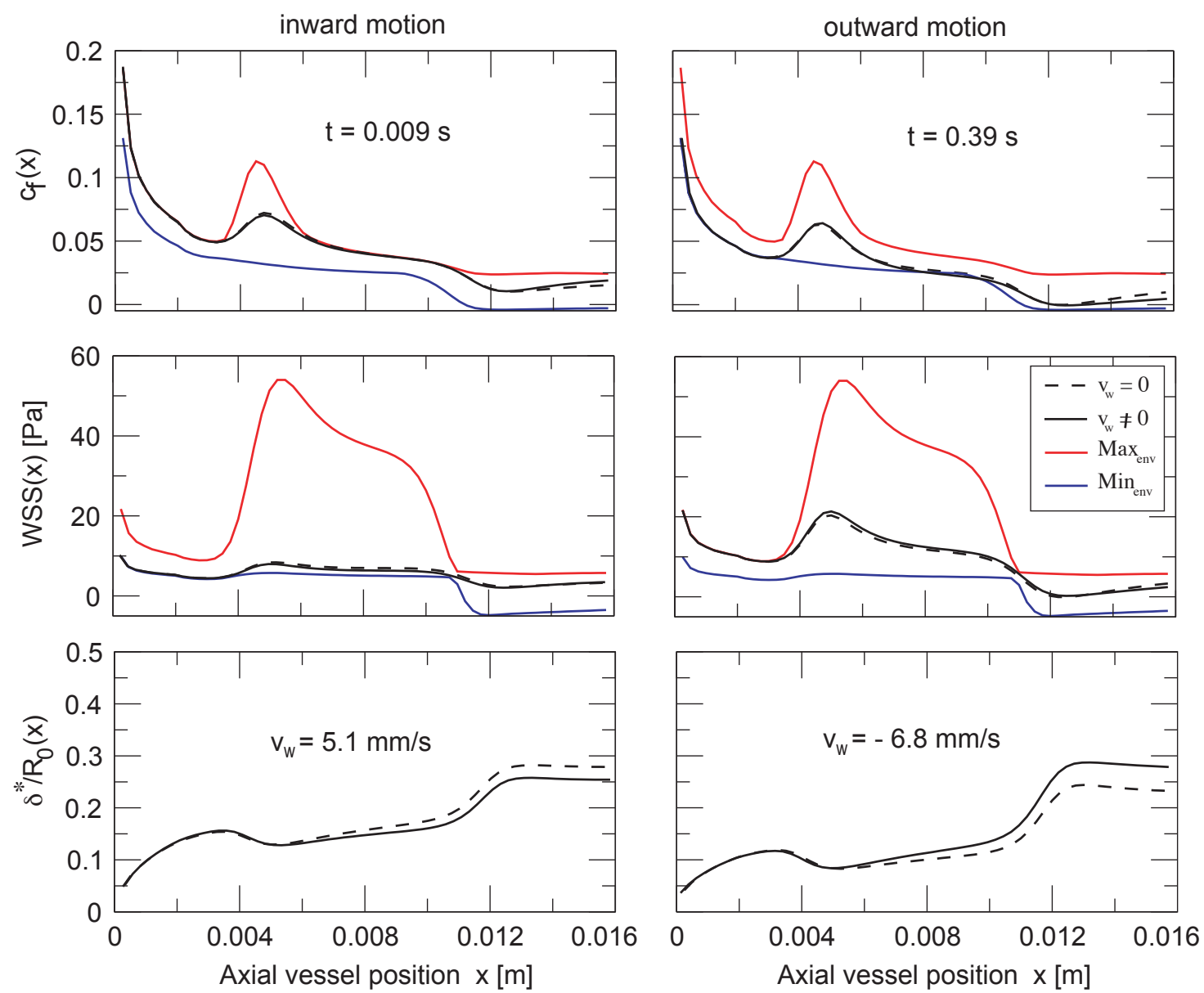

Figure 6.9: The bottom figure shows the normalised thickness of the boundary layer during in- (left) and outward motion (right) of the wall in the dynamic configuration presented in the third animation. Compared to zero wall velocity the boundary layer thickness in systole $\left(v_{w}=5.1 \mathrm{~mm} / \mathrm{s}\right)$ is increased in the entrance region of the deformation while it is decreased in the outlet region, in diastole $\left(v_{w}=-6.8 \mathrm{~mm} / \mathrm{s}\right)$ the situation is vice versa. The influence on viscous friction and wall shear stress is small and thus the difference in pressure loss over the deformation compared to $v_{w}=0$ is small $(\delta p \approx 1 \%$ of $\triangle p)$.

\subsubsection{Wall shear stress oscillation}

WALL shear stress oscillations for baseline conditions and under dobutamine challenge have been observed for various downstream locations. In fixed stenosis the amplitude of the oscillation is much smaller than in dynamic lesions, even though the peak values for fixed lesions are larger (compare envelopes in figure 6.10). 

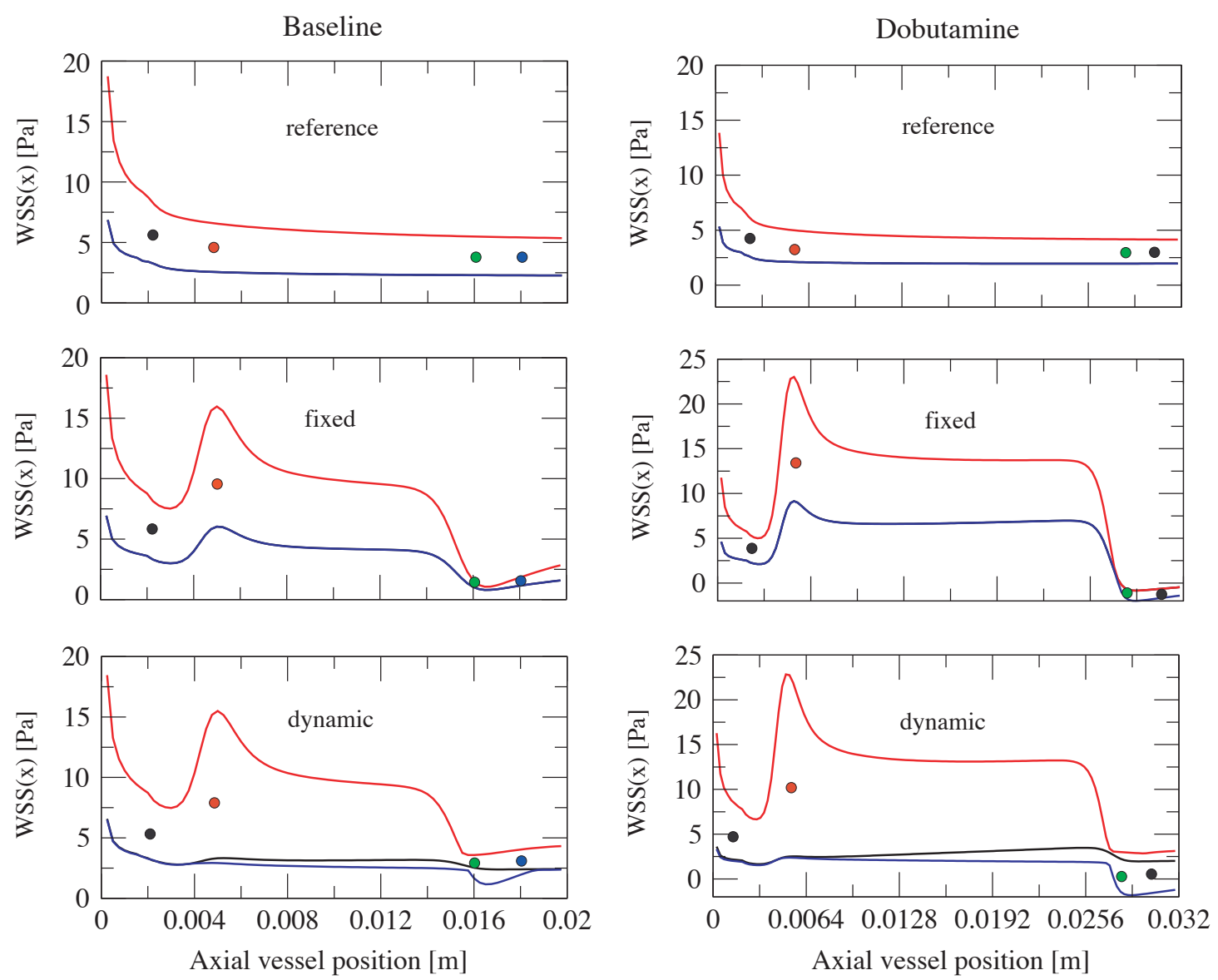

Figure 6.10: Axial wall shear stress distributions at baseline conditions (left) and under dobutamine challenge (right). In the deformation the amplitude of wall shear stress is larger in dynamic lesions (compare envelopes). Observation locations for the oscillations in figure 6.11 are given by coloured $\bullet$ 's.

As previously observed the vulnerable regions are in entrance region of the deformation and fade towards the end of the constriction. Eventually they become negative in the outlet region, as the flow separates (dobutamine). We have marked physiological relevant observation locations by coloured •'s. The amplitude of oscillation depends strongly on the axial position and the actual state of deformation (see figure 6.11). The wall shear stress oscillation is large in the entrance of the deformation, while it is small and negative in outlet regions where the flow tends to separate. The amplitudes in wall shear stress for fixed and dynamic lesions are 9.94 $\mathrm{Pa}, 12.58 \mathrm{~Pa}$ and 13.87 $\mathrm{Pa}, 20.46 \mathrm{~Pa}$ for baseline and dobutamine respectively. The peak values are 15.97 $\mathrm{Pa}, 15.50 \mathrm{~Pa}$ and $22.80 \mathrm{~Pa}, 22.83 \mathrm{~Pa}$ respectively. Values for the reference LAD are below $10 P a$ in both cases. 

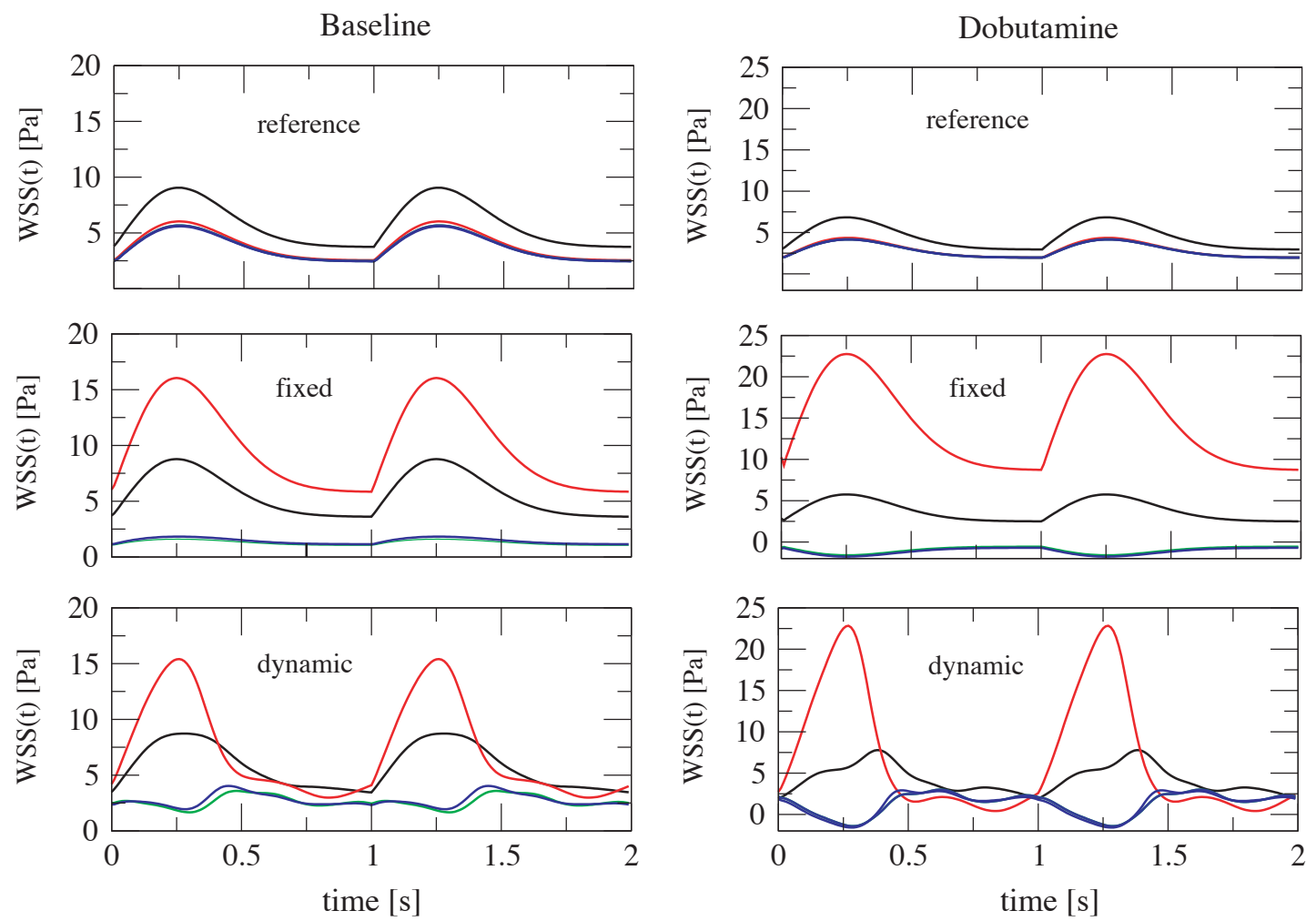

Figure 6.11: Wall shear stress oscillations for several axial positions where recorded at baseline conditions (left) and under dobutamine challenge (right). They are increased in the entrance of the deformation and may be negative in outlet regions where the flow separates.

\subsection{Human arterial tree}

THE general application of the averaged flow equations is to simulate the major arteries in the cardiovascular system $[140,59,23]$ and to understand vascular dysfunction's. The reason to investigate not only parts of the vasculature (like the coronary arteries) is because the boundaries of the network alter the haemodynamic conditions considerably and the choice for inflow is crucial. In contrast the inflow condition to the aorta can be well approximated by equation (4.18) (see figure 6.4). We applied an exponential flow wave with amplitude of $400 \mathrm{~cm}^{3} / \mathrm{s}$ and raising time of $t_{r}=0.25 \mathrm{~s}$ to the aorta, resulting in a total cardiac output (CO) of $4.893 \mathrm{l} / \mathrm{min}$. Due to deficiency of relations for the boundary layer across bifurcation's the frictional term in equation (3.27b) was modelled either by equation (3.55) (Hagen-Poiseuille flow) or by a linear relation for the Stokes boundary layer given in equation (3.62). The momentum correction coefficients were consequently modelled either by equation (3.56) or (3.63). 


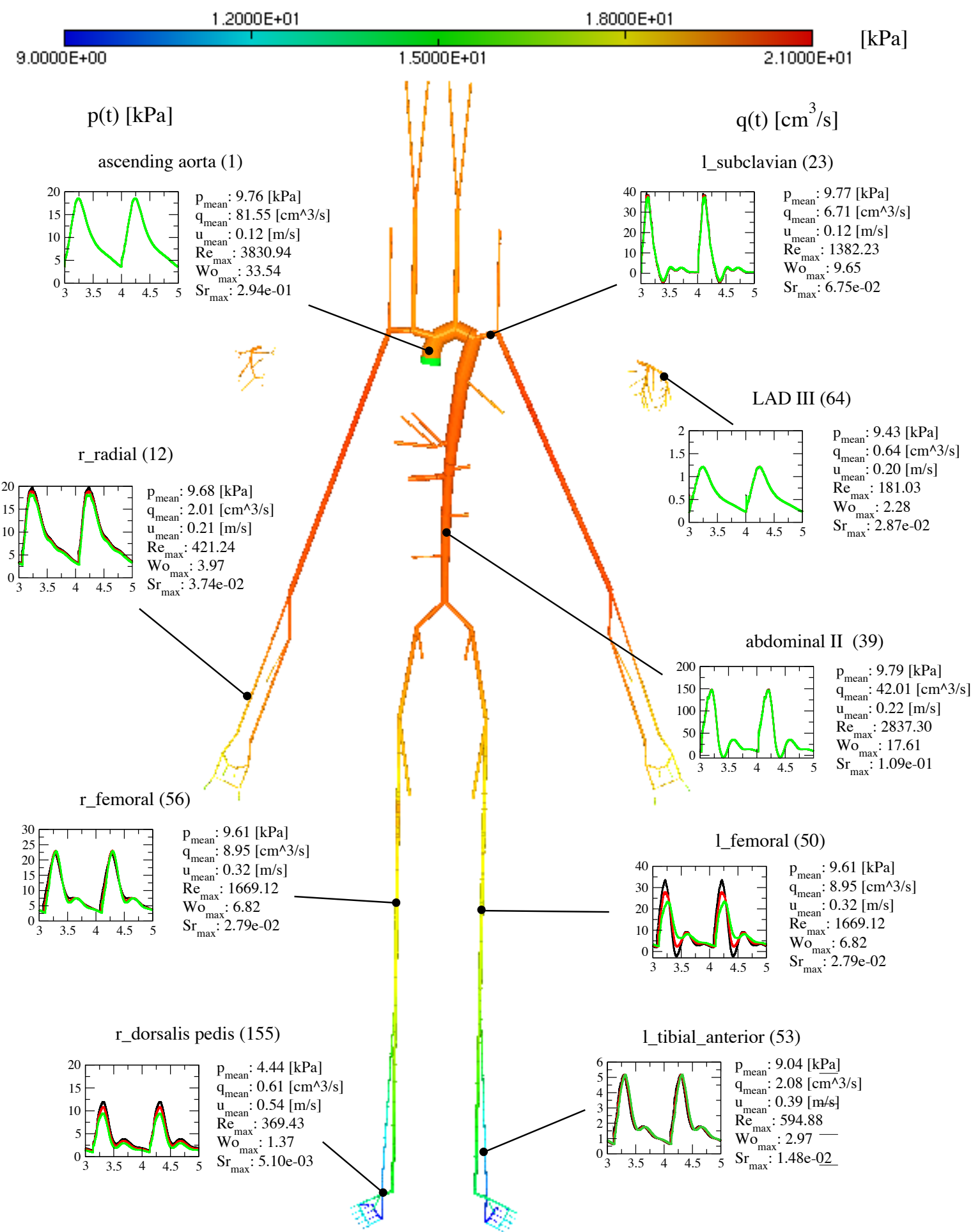

Figure 6.12: The illustration shows the major 175 arteries of the human cardiovascular system. The tubular entities are coloured according to the actual pressure distribution at $t=0.22 \mathrm{~s}$, the unit of the colour bar is $k P a$. The temporal dependence of pressure and flow waves at various locations are shown. 
THE results are based on the network topology mentioned in [20] and a topology of the major 48 coronary arteries presented by [139, 138]. The main palmar arteries of the hand and foot were included, whereas the dimensions and exact course are taken from [141, 142, 143, 144, 145]. The parameters for the major 175 arteries and there course are given in table A.1 and figure A.1 in the appendix. The windkessel parameters for the terminals of the network are found in table A.2. The simulation results are presented in figure 6.12. They show the pressure distribution, pressureflow waveforms and several mean quantities of the haemodynamic conditions in the network. We note that in the present 1D approximation the arterial tree is composed of tubular entities. The branching angles in figure 6.12 serve artistic purposes only.

\subsection{Left coronary arteries}

IN the following we use the flow conditions at the LMCA produced by the simulation of the arterial tree in the previous paragraph as a boundary condition to the LCA to determine the influence of a myocardial bridge in the mid LAD. We observe conditions were the phase and degree of the deformation function, the constriction length and the terminal resistance are varied in a physiological range, and we discuss potential flow limitation due to vessel collapse. The flow velocity, pressure, volume flow and deformation of the myocardial bridge are plotted in relation to a reference EKG (figure 6.13). The thick solid line in (IV) indicates the reference of a normal LAD having peak flow velocities of $30 \mathrm{~cm} / \mathrm{s}$, remaining dashed lines are values taken within the myocardial bridge. Published data from patient studies and experiments in [31, 32, 120] show good qualitative and quantitative agreement to our simulations.

\subsubsection{Pressure notch}

LINEARISED equations are only valid for small deformations, because the flow velocity is small in that case $\left(u_{s 2, s 4}<1 \mathrm{~m} / \mathrm{s}\right)$. However for stronger lumen reduction the flow velocity is significantly increased during deformation and hence the nonlinear term becomes more pronounced. This becomes evident in the characteristic pattern of flow velocity (velocity peak) and pressure (pressure notch) in figure 6.13 (I). The depth of the pressure notch increases with flow velocity and is therefore not persistent during phases of small deformation. Compared to the pressure of the normal LAD the depth of the notch is of the order $1.5 \mathrm{kPa} \leq \triangle p \leq 8 \mathrm{kPa}$ for diameter reductions between $81 \%$ and $96 \%$. For higher deformations ( $>98 \%$ ) the depth of the notch exceeds the intraluminal pressure, i.e. negative transmural pressure is observed, which would generally cause the vessel to collapse. We note that the present model is only valid if the pressure remains positive, which is satisfied for deformations smaller $98 \%$, however estimation of occlusion time may indicate the upper border of flow limitation. 
(I) severity

(II) time shift

(III) length

(IV)termination

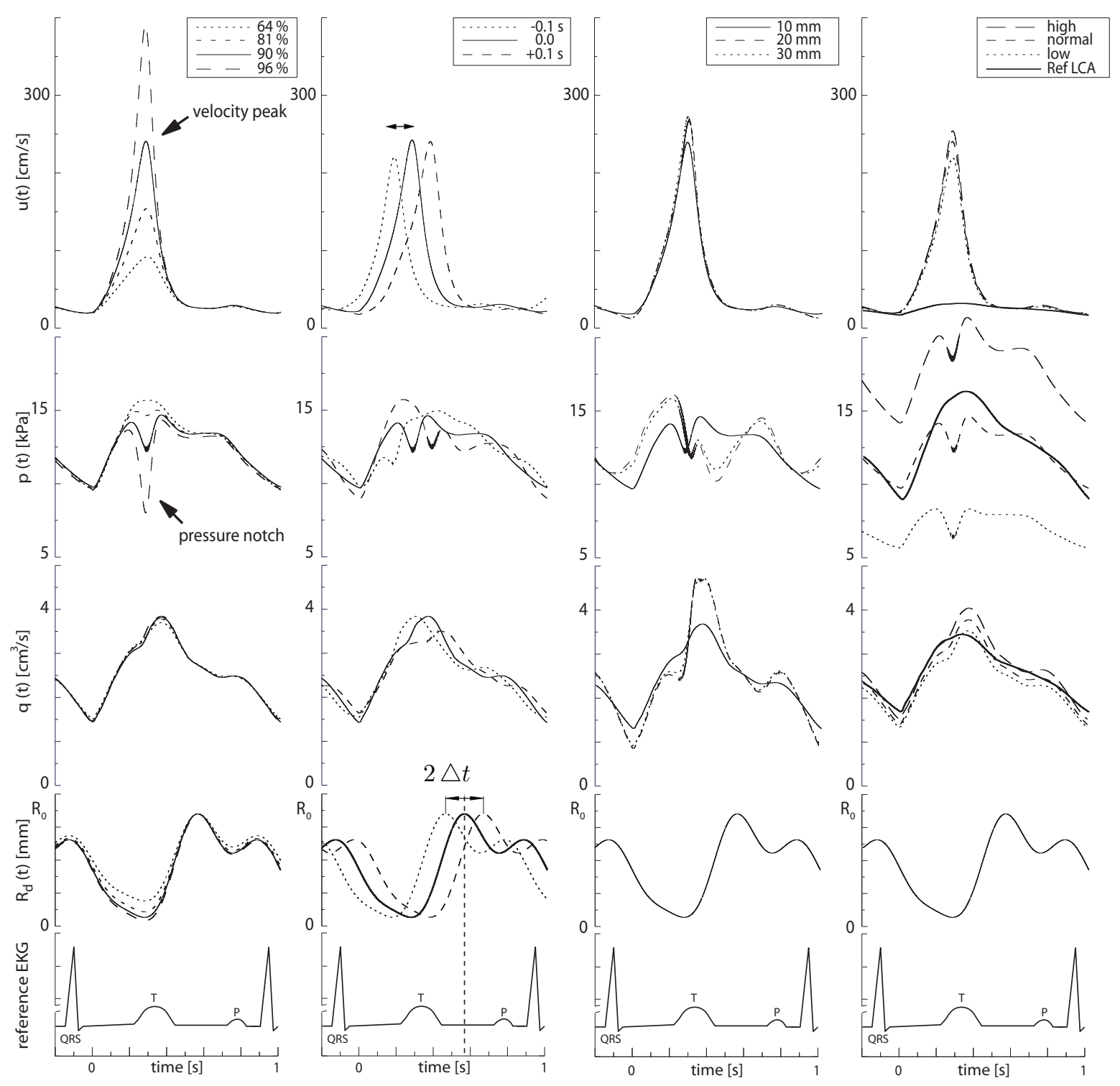

Figure 6.13: The flow velocity, pressure, volume flow and deformation of the myocardial bridge are plotted in relation to a reference EKG. Relaxation following asymmetric compression is delayed into diastole. The reference of a normal LAD (thick solid line in (IV)) is compared to the values taken within the myocardial bridge (dashed lines). In (I) we compare bridges of different severity. During deformation the peak flow velocity is increased (velocity peak) and in the nonlinear case a pressure notch is found. (II) illustrates the effect of time shifting the deformation with respect to the cardiac cycle. Further we change the length of the stenosis in (III) and the peripheral resistance in (IV). 


\subsubsection{Vessel collapse and reopening}

THE vessels in the circulatory system are collapsible under several circumstances: Firstly, the transmural pressure can cause significant collapse of the vessel especially in the downstream region, where the intraluminal pressure may drop below the extramural pressure $[65,66,67,68]$. In such cases, the flow through the tube is no longer dependent upon the difference in pressure between the inlet and outlet, but also on transmural pressure. At negative values the vessel walls cave in, further reduction may cause complete occlusion of the vessel. Due to frictional forces the flow velocity decreases and the kinetic energy will be converted to pressure, which again reopens the artery. Periodic vessel collapse and reopening is known as arterial flutter. Secondly, elevated flow conditions in severe stenosis may reduce transmural pressure, so that the stenosis will collapse [69]. This results in additional flow choking. The critical degree of stenosis where the stenosis will collapse depends on flow rate. As the results in the previous section, it was shown in [69] that the reduction of local pressure is more than $7.5 \mathrm{kPa}$ in a $80 \%$ stenosis and that the flow will be choked at a velocity of $3 \mathrm{~m} / \mathrm{s}$. Thirdly, increased eccentricity caused by cyclic deformation of the vessel increases the likelihood of collapse, because the buckling pressure is temporally reduced. This situation may contribute significantly to flow limitation, so that we will shortly discuss the expected influence. From dynamic simulations we see that severe deformations are present between $0.2 s$ and $0.4 s$. If the deformation during this period is above $80 \%$, elevated flow conditions may cause temporal vessel collapse, which limits the total flow by up to $20 \%$. We note that we have neglected the flexural rigidity in the vessel wall, so that the vessel collapses significantly earlier, i.e as the transmural pressure equals zero. These values are just estimations for the upper limit in flow chocking and may reduce by the introduction of bending resistance of the vessel wall.

\subsubsection{Flow velocity pattern}

To reproduce the characteristic flow velocity pattern found in myocardial bridges we have used a set of parameters given in [31]. The intracoronary Doppler flow velocity pattern given in figure 6.14 was taken within a myocardial bridge. The maximum peak flow velocity in the measurements lies in the range of $u_{s 2, s 4}=85 \mathrm{~cm} / \mathrm{s}-105 \mathrm{~cm} / \mathrm{s}$. The peak flow velocities in our simulations show good quantitative agreement to the measurements. They are $u_{s 1}=24.2 \mathrm{~cm} / \mathrm{s}$ proximal, $u_{s 2, s 4}=86 \mathrm{~cm} / \mathrm{s}$ within, and $u_{s 5}=26.5 \mathrm{~cm} / \mathrm{s}$ distal the bridge. Besides the peak flow velocities the Doppler flow velocity tracing within a myocardial bridge is characterised by an abrupt early diastolic flow acceleration, a rapid mid-diastolic deceleration, and a mid-to-late-diastolic plateau [120]. The mean flow velocity pattern obtained by the simulation agrees well with the in vivo measurements (see overlay in figure 6.14). The acceleration of the fluid is caused by rapid reduction of the cross-sectional area of the vessel during the 
contraction phase, while a rapid deceleration is caused during relaxation. By comparison to static stenosis we found that the anomalous accelerations are dependent on the phase and degree of the deformation function. Maximum peak flow velocities are observed for deformation gradients having opposite phase with respect to the fluid acceleration caused by the pressure gradient, i.e. for maximum deformation during the systole.

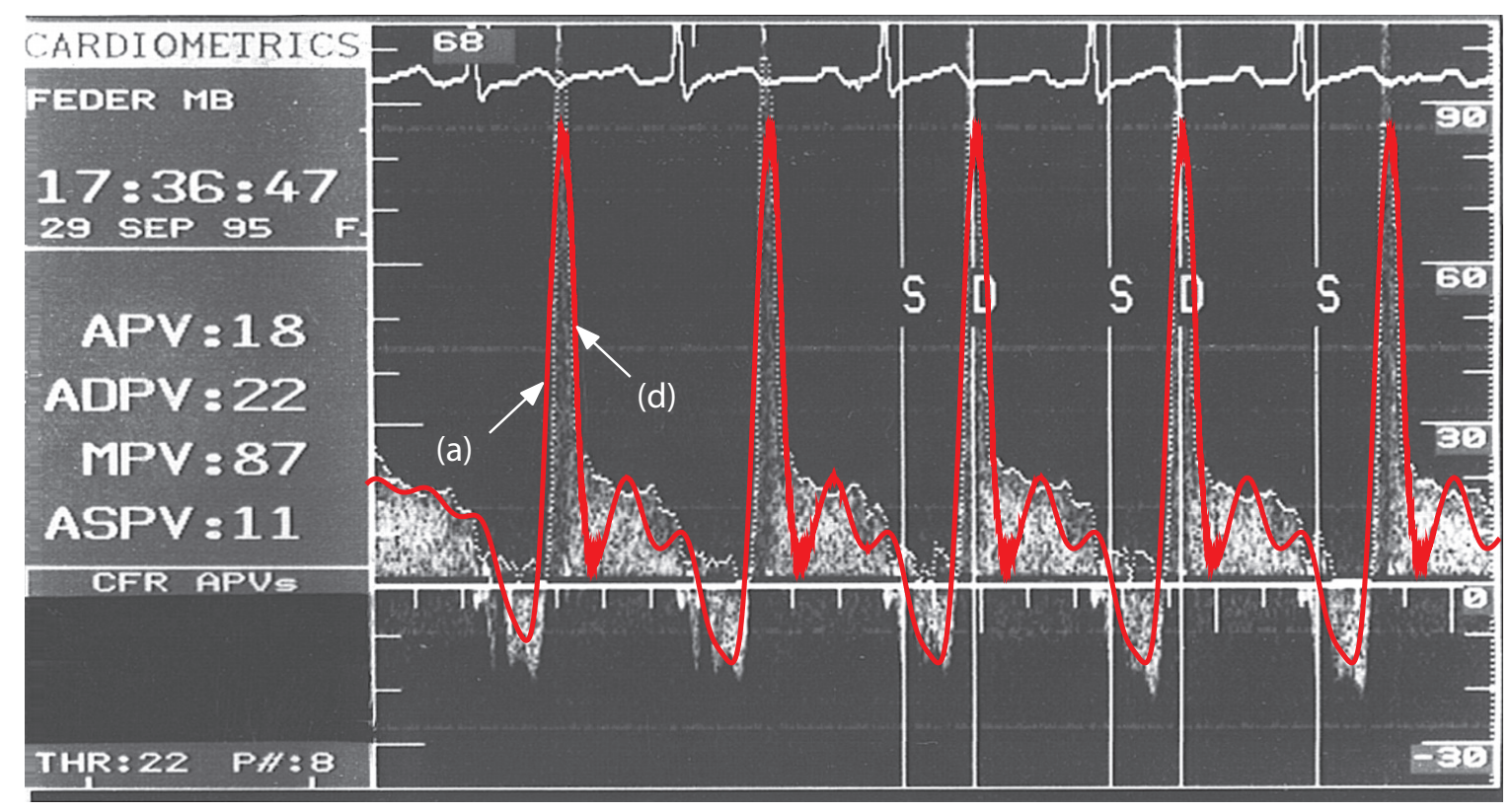

(a) compression

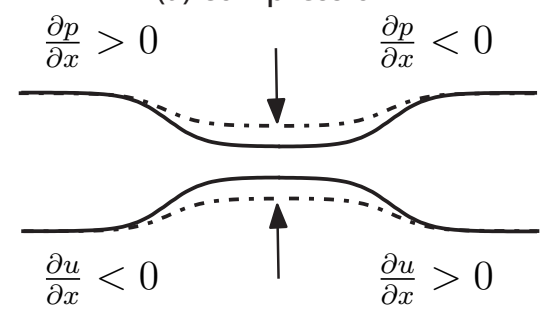

(d) relaxation

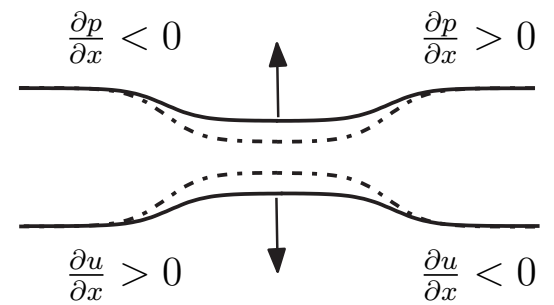

Figure 6.14: Typical intracoronary Doppler flow velocity profile obtained within a myocardial bridge of left anterior descending coronary artery (top). Abrupt diastolic flow velocity acceleration is followed by mid-diastolic plateau and retrograde systolic flow. APV indicates average peak flow velocity; ADPV, average diastolic peak flow velocity; MPV, maximal peak flow velocity; ASPV, average systolic peak flow velocity; $\mathrm{S}$, systole; and $\mathrm{D}$, diastole. All values are $\mathrm{cm} / \mathrm{s}$. (modified from [31]) The mean velocity obtained from the simulation is overlaid as red curve. We have indicated the phases of acceleration by (a) and that of deceleration by (d). Further we show the additional pressure and velocity gradients caused by the deformation (bottom). 
SECONDARY fluid flow during the deformation is caused by momentum transfer between solid and fluid. The amount of fluid displaced depends on the normal diameter and length of the bridge, and the degree of the deformation. In the case that this additional fluid motion exceeds the normal flow, reverse flow at the proximal end of the bridge is observed during inward motion of the wall, while the like for the distal end is observed during relaxation.

\subsubsection{Deformation time shift}

A pressure-flow wave typically requires $20 \mathrm{~ms}$ to travel the distance between the aortic valve and the myocardial bridge. In contrast the deformation of the vessel during systole happens instantaneously. The pressure in the left ventricle however has to overcome the aortic pressure to allow wave ejection, typically this time-span is $80 \mathrm{~ms}$. The phase of deformation with respect to the wave entering the muscle bridge is therefore dependent on the relative distance to the aortic valve and the time required by blood compression in the left ventricle. This however strongly depends on anatomy, so that we investigate the pressure-flow patterns for deformations shifted in time by $\triangle t= \pm 0.1 \mathrm{~s}$ (figure 6.13 (II)). Another reason to investigate time shifted deformations is that a large amount of blood volume is transferred during diastole. The characteristic pattern of flow acceleration and deceleration (velocity peak and pressure notch) change their position with deformation phase. The maximum peak flow velocities are found if the deformation has opposite phase with respect to the flow wave.

\subsubsection{Segment length}

As before the segment length of the myocardial bridge was modified (see figure 6.13 (III)). We note that in contrast to previous simulations we have assumed HagenPoiseuille flow. On the basis of anatomic relevant values we used $10 \mathrm{~mm}, 20 \mathrm{~mm}$ and $30 \mathrm{~mm}$. It was found that the peak flow velocity increases with segment length and that the peak volume flow is significantly increased during relaxation. This indicates that peristaltic transport increases as the segment length is increased. For reasons that the phase of deformation was fixed in this case, we may expect that variation will lead to either drawing or blowing peristaltic transport.

\subsubsection{Peripheral resistance}

FINALLY in figure 6.13 (IV) we have shown the influence of peripheral resistance on flow conditions in a myocardial bridge. The variation was observed in three categories $\left(\triangle R_{t}= \pm 30 \%\right)$ for low, normal and high peripheral resistance. We found that the peak flow velocity, volume flow and mean pressure increase with peripheral resistance. 


\section{Chapter 7}

\section{Discussion, Conclusion and Outlook}

T $\mathrm{N}$ this final chapter, we summarise the main results in reference to their convenience $\mathbf{I}$ in medicine, and we discuss possible future work, either applications in medicine or enhancements to the boundary layer model.

\subsection{Summary of the results}

ONE of the most important results of this study is the development of a transient boundary layer model for arterial blood flow, which allows to calculate the wall shear stress, viscous friction and momentum correction in dependence on time and axial position. Clinical relevant results of separating flow conditions in the entrance region of a time dependent non-uniform vessel geometry (myocardial bridge) in the left coronary arteries were obtained by numerical simulation. The boundary layer method reproduced several important features of myocardial bridges, which clarify recent doubts in the field of study. Besides the clinical findings the method could be used as the basis for one-dimensional network simulations in arterial blood flow, because reasonable approximations for viscous friction and momentum correction are directly available. In the following we shortly summarise the major results before we give a final conclusion.

\section{Flow limitation and pressure drop}

WE found that in developing flow conditions the FFR is overestimated by the assumption of Hagen-Poiseuille flow, and, as expected, that the mean FFR in dynamic lesions is much larger than in fixed stenotic environment, because the distal pressure recovers during relaxation phase. Consequently the pressure drop and flow reduction across fixed stenoses are more pronounced than in myocardial bridges. As in [27, 34] 
we found that the pressure proximal to the deformation is increased if the degree of deformation or the length of deformation is increased. At baseline conditions the mean translesional pressure drop was $\triangle p=1.25 \mathrm{kPa}$, which compares well with the value of measurements in [40], where $\triangle p=1.19 \mathrm{kPa}$. Under dobutamine challenge the pressure drop was $\triangle p=1.54 k P a$, which however is close to the measured value of $\triangle p=1.85 \mathrm{kPa}$. Despite the deviation the values for the mean pressure derived FFR agree favourably with the measurements mentioned above, where the values for baseline and dobutamine challenge were 0.90 and 0.84 , respectively.

\section{Series stenoses}

THE simulations indicate that the pressure drop of series stenoses in developing flow conditions cannot be obtained by a summation of pressure drops for single stenoses, since the proximal and distal stenosis influence each other. Therefore several consecutive stenoses along the same epicardial artery require separate determination of stenosis severity. We found that when two stenoses are close together, the pressure drop is approximately equal to that of a stenosis with twice the length of a single stenosis. However, when the stenoses are separated by more than the length of the stenosis the downstream deformation is generally dominant in wall shear stress and frictional forces as well as in the extent of the post-stenotic separation zone, because the core flow velocity is increased, even though the entrance flow conditions are preserved. Further, it is likely that a downstream stenosis with equal deformation collapses significantly earlier, because the core flow velocity is increased, so that the transition from subcritical flow $(S<1)$ to supercritical flow $(S>1)$ happens earlier.

\section{Wall shear stress}

In steady flow conditions the location of maximum wall shear is always upstream the neck of the stenosis, and moves upstream as the Reynolds number increases. Generally the maximum is at the entrance of the stenosis and fades towards the end of the stenosed section. Eventually it becomes negative after separation of the boundary layer. In series stenoses the wall shear stress is significantly increased in the distal stenosis. Consequently the second stenosis can be seen as the more vulnerable, in wall shear stress, frictional forces and consequently in flow limitation. Compared to wall shear stresses in non-diseased vessels $\left(5-10 \mathrm{~N} / \mathrm{m}^{2}\right)$ vulnerable regions are endothelial cells in the throat of a strong deformation. They may experience wall shear stresses in excess of $60 \mathrm{~N} / \mathrm{m}^{2}$.

\section{Flow separation}

THE boundary layer separation in the post-stenotic region indicates an emerging top-hat velocity profile from the stenosis, with sideway counter-current flows. The 
decay in core flow velocity indicates shear widening of the top-hat until reattachment. It was found that the distance over which the outlet effect occurs is smaller for stenosis with small deformation and small Reynolds number. The extent of the recirculation zone is primarily dependent on vessel deformation and Reynolds number. The separation point moves upstream, while the reattachment point moves downstream if the Reynolds number or deformation increases. However, we found that the extent also correlates with the length of the constriction. In other words vessels with the same degree of stenosis, but with the stenosis having different curvatures and lengths, have recirculation regions that differ markedly in their extent.

DURING a cyclic compression of the artery the separation point moves slightly upstream, while the reattachment point of the boundary layer moves farther downstream. A particularity of series stenoses is that the extent of the recirculation zone in the interconnecting segment is reduced. This is due to early reattachment caused by fluid acceleration in the converging part of the distal stenosis. The non-linear term is pronounced in the separation region, while it is close to unity in converging regions. We note that the momentum correction becomes markedly smaller than before the upstream stenosis (below unity). This indicates that the entrance profile into the second stenosis is almost flat, but has increased core velocity, while the counter current flow at the wall has disappeared.

\section{Influence of wall velocity}

COMPARED to fixed deformations $\left(v_{w}=0\right)$ the boundary layer thickness in systole is increased in the entrance region of the deformation, while it is decreased in the outlet region, and the situation is vice versa in diastole. In fact this is due to an additional pressure gradient, which causes fluid acceleration or deceleration depending on axial position in the constriction and whether the wall moves inwards or outwards. For example during inwards motion of the wall the fluid is decelerated at the entrance and accelerated at the outlet of the deformation, while the situation is opposite if the vessel wall moves outwards. Due to the symmetry of the indentation there is no additional acceleration or deceleration in the centre of the deformation. The influence of the wall velocity onto the boundary layer properties is more pronounced if $\frac{v_{w}}{V}$ is reasonably large, i.e. if the deformation that the axial flow experiences during passage of the constriction is comparable to the radius of the tube. The influence of wall velocity on viscous friction and wall shear stress is small and thus the difference in pressure loss over the deformation with $v_{w} \neq 0$ compared $v_{w}=0$ is small $(\delta p \approx 1 \%)$.

\section{Vessel collapse}

FLOW limitation due to vessel collapse is possible if the intraluminal pressure falls below the extramural pressure. The characteristic flow velocity acceleration and deceleration in myocardial bridges, i.e. the velocity peak causes congruent pressure drop, 
i.e. a pressure notch. The depth of the pressure notch increases with flow velocity and is therefore not persistent during phases of small deformation. Compared to the pressure of the normal LAD the depth of the notch is of the order $1.5 \mathrm{kPa} \leq \triangle p \leq 8 \mathrm{kPa}$ for diameter reductions between $81 \%$ and $96 \%$. For higher deformations $(>98 \%)$ the depth of the notch exceeds the intraluminal pressure, i.e. negative transmural pressure is observed, which would generally cause the vessel to collapse. We found that if elevated flow conditions are present during one fifth of the pulsatile cycle, temporal vessel collapse may limit the total flow by up to $20 \%$. To obtain these estimates we have neglected the flexural rigidity of the vessel wall, so that the vessel collapses significantly earlier, i.e as the transmural pressure equals zero. These values are just estimations for the upper limit in flow chocking and may reduce by the introduction of bending resistance of the vessel wall.

\subsection{Discussion}

THE results have demonstrated that the formation and development of flow separation and reattachment in the post-stenotic region of a time dependent constriction are very complicated, especially secondary fluid motion in the systolic deceleration phase can cause situations of reverse flow. We found that the post-stenotic flow is influenced by a number of factors, including the degree of stenosis, the flow and deformation waveform, and the geometry of the constriction. The calculations in the previous chapter suggest that the degree of deformation of the stenosis alone does not adequately characterise the flow through myocardial bridges, and that geometric and physiological features such as the curvature, extent, and asymmetry of the stenosis, and the shape of the pulsatile waveform have substantial effects on the haemodynamic conditions. The variety of model parameters complicates the analysis and convenient simplification was required. We found that the major contribution to the losses across myocardial bridges is caused by viscous forces, so that the total perfusion to the myocardium is strongly dependent on the Reynolds number, the severity and length of the muscle bridge. The separation vortex in the post stenotic region, however, has small influence for observed deformations, so that the accurate determination of wall shear stress and consequently viscous friction leads to reasonable results for the pressure drop. At severe deformations further examination is required to determine if the turbulent character of the flow increases percentage contribution to the losses.

VARIOUs steady and unsteady numerical simulations were performed, all yielding excellent agreement with available experimental data. The predicted values of fractional flow reserve in dynamic lesions agree well with the clinical findings in [40], however, further quantification in more defined geometries is required. We could also confirm the velocity peak found in qualitative analysis of the Doppler flow profiles within the myocardial bridge [31]. The velocity pattern is characterised by an abrupt early diastolic flow acceleration, a rapid mid-diastolic deceleration, and a mid-to-late- 
diastolic plateau [120]. The acceleration of the fluid is caused by rapid reduction of the cross-sectional area during the contraction phase, while a rapid deceleration is caused during relaxation. By comparison to static stenosis we found that the anomalous accelerations are dependent on the phase and degree of the deformation function. Maximum peak flow velocities are observed for deformation gradients having opposite phase with respect to the fluid acceleration caused by the pressure gradient, i.e. for maximum deformation during the systole. Anyhow we found that the peak pressure values are not consistent with the findings in [31], where a high pressure chamber in the centred segment $\left(\Omega_{3}\right)$ of a series stenoses was observed. We suppose that the pressure catheter was entrapped during the measurement or that this effect is only observed for total occlusion or collapse of the muscle bridge.

REGARDING the wall shear stresses and the development of atherosclerosis the findings are consistent with [27], where the proximal segment is more susceptible to the development of atherosclerotic lesions. As previously noted [34] found that the pressure proximal to the myocardial bridge was higher than the aortic pressure, and concluded that the disturbance of blood flow and high wall shear stress proximal to the myocardial bridge was the main contributor to the development of atherosclerosis in the proximal segment. The observed wall shear stress distributions in this study indicate that the proximal segment is more susceptible to the development of atherosclerosis, firstly because the pressure is increased there and secondly because the wall shear stress oscillations are maximum in the entrance region of the deformation. In contrast bridged segments are relatively spared, because the wall shear stress fades towards the end of the deformation. In a series of myocardial bridges it is likely that the segment between the deformations and distal to the myocardial bridge are protected from atherosclerosis, because the wall shear stress is very low and negative in separated flow regions.

DuE to the assumptions made in the boundary layer model, the approximation fails for the prediction of reverse flow and flow where the boundary layers merge or detach, i.e. fully developed flow. Under these aspects severe deformations cannot be calculated, because the boundary layers merge and detach during compression and relaxation of the vessel respectively. Further, the length of the computational domain is restricted by the entrance length, which depends on Reynolds number. The model also fails to describe conditions where the transmural pressure becomes negative, i.e. flow limitation due to vessel collapse. And finally the pulsatile frequency and the frequency of wall motion has to be sufficiently low (a few $H z$ ), so that the approximation of quasi-stationary evolution of the boundary layers is satisfied.

\subsection{Conclusion}

In the present study we have presented a method for simulation of unsteady blood flow in a time dependent vessel geometry using an integral boundary layer method. 
The strong interaction of the viscous boundary layer and the inviscid core flow proposed by Veldman [83, 84] models the pressure and the extent of the separation region under the assumption of self-similar Falkner-Skan flow profiles. The equations were modified to the flow situation under consideration, capabilities and shortcomings of the method were discussed. Numerical simulations were performed for idealised stenosis geometries with a time dependent, smooth wall contour, but with a physiologically realistic coronary artery flow waveform. It was demonstrated that the proposed method is able to reproduce several important features found in myocardial bridges. The results indicate that physiological consequences may be reproduced also in a clinical setting, however, this remains to be confirmed by in vivo studies. The comparison of our findings with the published data from patient studies in $[120,40]$ supports a potential clinical relevance of our simulation. Moreover, the model was found to be a robust and accurate numerical method to simulate a variety of entrance flow conditions in the cardiovascular system. Besides the advantage of computational time taken for the simulation, the choice of parameters, such as location, length and severity of the lesion are easily determined by coronary angiography. Clinical application may reduce invasive intervention, because the flow limiting effect of different haemodynamic conditions is easily calculated from aortic pressure conditions.

\subsection{Outlook}

THE presented boundary layer model for one-dimensional arterial blood flow opens several possibilities for future research. Those are situated in either model validation in defined fluid dynamical conditions (or in vivo), or enhancement of the present model by the formulation of branching conditions to represent vascular networks. The former is followed by diagnostic and clinical activities, i.e. patient specific modelling of myocardial bridges, while the latter enables realistic one-dimensional modelling of losses and wall shear stresses across bifurcations. On that account the method should comprise the following additional features: (i) possibility to simulate fully developed flow conditions, i.e. merging boundary layers, so that the entrance length or severity of the deformation do not restrict the computations, (ii) extension of the boundary layer approach by time dependent similarity solutions may improve the model at high Womersley and Strouhal numbers, because a time dependent similarity solution of the boundary layer equations includes the Stokes layer, (iii) branching conditions for boundary layer properties at bifurcations, (iv) other types of flows like sources and sinks, i.e. blowing/sucking of the boundary layer, (v) approximation of flexural rigidity of the vessel wall by a non-linear pressure area relation would address losses due to vessel collapse at severe deformations, (vi) shear dependent viscosity may improve predictions of the wall shear stress and consequently frictional losses and finally (vii) inclusion of a turbulence model may extend the range of validity at severe deformations. 


\section{Bibliography}

[1] Reyman HC: Disertatio de vasis cordis propriis. PhD thesis, University Göttingen 1737.

[2] Prandtl L (Ed): Über Flüssigkeitsbewegung bei sehr kleiner Reibung, Verh. III. Int. Math. Kongr., Heidelberg: Teubner Verlag Leipzig 1905.

[3] Womersley J: Flow in the larger arteries and its relation to oscillating pressure. J Physiol 1954, 124:31-32.

[4] Womersley J: Oscillating flow in arteries: effect of radial variation in viscosity on the rate of flow. J Physiol 1955, 127:38-39.

[5] Womersley J: Method for the calculation of velocity rate of flow and viscous drag in arteries when the pressure gradient is known. J Physiol 1955, 127:553-563.

[6] Womersley J: Oscillatory motion of viscous liquid in a thin-walled elastic tube: I. The linear approximation for long waves. Phil Mag 1955, 46:553-563.

[7] Lighthill J: Waves in Fluids. Scientific Computation, Cambridge Mathematical Library 2001.

[8] Bergel DH: Cardiovascular Fluid Dynamics I. Academic Press London 1972.

[9] Bergel DH: Cardiovascular Fluid Dynamics II. Academic Press London 1972.

[10] Bertram C: Biological Fluid Dynamics. Springer-Verlag Berlin 1995.

[11] Caro CG, Pedley TJ, Schroter RC, Seed WA: The mechanics of the circulation. Oxford University Press 1978.

[12] Dinnar U: Cardiovascular Fluid Dynamics. CRC Press Inc. 1981.

[13] Fung YC: Biodynamics Circulation. Springer-Verlag New York 1984.

[14] Noordergraaf A: Circulatory System Dynamics. Academic Press London 1978. 
[15] Pedley TJ: The fluid dynamics of large blood vessels. Cambridge University Press 1980.

[16] Zamir M: The Physics of Pulsatile Flow. Biological Physics Series, SpringerVerlag Heidelberg 2000.

[17] Formaggia L, Lamponi D, Tuveri M: Numerical Modelling of 1D Arterial Networks Coupled with Lumped Parameters Description of the Heart 2000 .

[18] Olufsen MS: Structured tree outflow condition for blood flow in larger systemic arteries. Am Physiol Soc 1999, 45:H257-H268.

[19] Olufsen MS, Nadim A: On deriving lumped modells for blood flow and pressure in systemic arteries. Math Biosci Eng 2004, 1:61-80.

[20] Olufsen MS: Modeling the arterial System with Reference to an Anestesia Simulator. PhD thesis, IMFUFA Roskilde University 1998.

[21] Peterson LH: The Dynamics of Pulsatile Blood Flow. Circ Res 1954, 2(127-139).

[22] Sherwin S, Formaggia L, Peiro J, Franke V: Computational modelling of 1D blood flow with variable mechanical properties and its application to the simulation of wave propergation in the human arterial system. Int J Numer Meth Fluids 2003, 43:673-700.

[23] Sherwin SJ, Franke V, Peiro J: One-dimensional modelling of a vascular network in space-time variables. J Eng Math 2003, 47:217-250.

[24] Westerhof N, Bosman, DeVries CJ, Noordergraaf A: Pressure and flow in the systemic arterial system. J Biomech 1972, 5:629-641.

[25] Westerhof N, Elzinga G, Sipkema P: An artificial arterial system for pumping herarts. J Appl Physiol 1971, 31:776-781.

[26] Westerhof N, Bosman F, De Vries CJ, Noordergraaf A: Analog studies of the human systemic arterial tree. J Biomech 1969, 2:121-143.

[27] Alegria JR, Herrmann J, Holmes DR, Lerman A, Rihal CS: Myocardial bridging. Eur Heart $J$ 2005, 26:1159-1168.

[28] Bourassa MG, Butnaru A, Lesperance J, Tardif JC: Symptomatic Myocardial Bridges: Overview of Ischemic Mechanisms and Current Diagnostic and Treatment Strategies. J Am Coll Cardiol 2003, 41(3):351-359. 
[29] Angelini P, Tivellato M, Donis J: Myocardial Bridges: a review. Prog Cardiovasc Dis 1983, 26:75-88.

[30] de Winter RJ, Kok WEM, Piek JJ: Coronary atherosclerosis within a myocardial bridge, not a benign condition. Heart 1998, 80:91-93.

[31] Klues H, Schwarz E: Disturbed intracoronary hemodynamics in myocardial bridging. Circ 1997, 96(9):2905-2913.

[32] Möhlenkamp S, Hort W, Ge J, Erbel R: Update on Myocardial Bridging. Circ 2002, 106(20):2616-2622.

[33] Herrmann J, Higano ST, Lenon RJ, Rihal CS, Lerman A: Myocardial bridging is associated with alteration in coronary vasoreactivity. Eur Heart $J$ 2004, 25:2134-2142.

[34] Ge J, Jeremias A, Rupp A, Abels M, Baumgart D, Lui F, Haude M, Görge G, von Birgelen C, Sack S, Erbel R: New signs characteristic of myocardial bridging demonstrated by intracoronary ultrasound and Doppler. Eur Heart J 1999, 20:1707-1716.

[35] White CW, Wright CB, Doty DB: Does visual interpretation of the coronary arteriogram predict the physiological importance of a coronary stenosis? $N$ Engl J Med 1984, 310:819-824.

[36] Vogel RA: Assessing stenosis significance by coronary angiography. Are the best variables good enough? J Am Coll Cardiol 1988, 12:692-693.

[37] De Bruyne B, Bartunek J, Sys S: Simultaneous coronary pressure and flow velocity measurements in humans. Circ 1996, 94:1842-1849.

[38] Pijls NHJ, Van Gelder B, Van der Voort P: Fractional flow reserve: a useful index to evaluate the influence of an epicardial coronary stenosis on myocardial blood flow. Circ 1995, 92:183-193.

[39] De Bruyne B, Pijls N, Heyndrickx GR, Hodeige D, Kirkeeide R, Gould L: Pressure-Derived Fractional Flow Reserve to Assess Serial Epicardial Stenoses. Circ 2000, 101:1840-1847.

[40] Escaned J, Cortes J, Flores A, Goicolea J: Importance of Diastolic Fractional Flow Reserve and Dobutamine Challenge in Physiologic Assessment of Myocardial Bridging. J Am Coll Cardiol 2003, 42(2):226-233.

[41] Pijls NHJ, De Bruyne B, Peels K, Van der Voort P, Bonnier HJRM, Bartunek J, Koolen JJ: Measurement of Fractional Flow Reserve to asses the functional severity of Coronary-Artery Stenoses. N Engl J Med 1996, 334(26):1703-1708. 
[42] Siebes M, Chamuleau SAJ, Meuwissen M: Influence of hemodynamic conditions on fractional flow reserve: parametric analysis of underlying model. Am J Physiol Heart Circ Physiol 2002, 283:H1462 - H1470.

[43] Pijls NHJ, De Bruyne B, Smith L, Aarnoudse W, Barbato E, Bartunek J, Bech GJW, van de Vosse FN: Coronary Thermodilution to Assess Flow Reserve. Validation in Humans. Circ 2002, :2482-2486.

[44] Hau WK: Fractional flow reserve and complex coronary pathologic conditions. Eur Heart $J$ 2004, 25:723-727.

[45] Long Q, Xu XY, Ramnarine KV, Hoskins P: Numerical investigation of physiologically realistic pulsatile flow through arterial stenosis. $J$ Biomech 2001, 34:1229-1242.

[46] Tu C, Deville M, Dheur L, Vanderschuren L: Finite Element Simulation of Pulsatile Flow through Arterial Stenosis. J Biomech 1992, 25(10):11411152.

[47] Payne SJ: Analysis of the effects of gravity and wall thickness in a model of blood flow through axisymmetric vessels. Med Biol Eng Comput 2004, 42:799-806.

[48] Canic S, Kim EH: Mathematical analysis of the quasilinear effects in hyperbolic model of blood flow through compliant axi-symmetric vessels. Math Meth Appl Sci 2003, 26(14):1161-1186.

[49] Barnard ACL, Hunt WA, Timlake WP, Varley E: A Theory of Fluid Flow in Compliant Tubes. Biophys J 1966, 6:717-724.

[50] Quarteroni A, Formaggia L: Handbook of Numerical Analysis: Computational Models for the Human Body. Elsevier Amsterdam 2004.

[51] Drzewiecki G, Field S, Moubarak I, Li JKJ: Vessel growth and collapsible pressure-area relationship. Am J Physiol 1997, 42:H2030-H2043.

[52] A two-Layer Model of the Static Behaviour of Blood Vessel Walls. 2004.

[53] Veress AI, Vince DG, Anderson PM, Cornhill JF, Herderick EE, Klingensmith JD, Kuban BD, Greenberg NL, Thomas JD: Vascular mechanics of the coronary artery. Z Kardiol 2000, 89(2):II/92-II/100.

[54] Vito RP, Dixon SA: Blood Vessel Constitutive Models-1995-2002. Annu Rev Biomed Eng 2003, 5:413-439. 
[55] Remington JW, Wood EH: Formation of Peripheral Pulse Contour in Man. J Appl Physiol 1956, 9:433-442.

[56] Berger DS, Li JKJ, Noordergraaf A: Differential effects of wave reflections and peripheral resistance on aortic blood pressure: a model-based study. Am J Physiol 1994, 266:H1626-H1642.

[57] Stergiopulos N, Westerhof BE, Westerhof N: Total arterial inertance as the fourth element of the windkessel model. Am J Physiol 1999, 276:H81H88.

[58] Wang TJ, Flewitt JA, Shrive NG, H PK, Tyberg JV: Systemic venous circulation. Waves propagating on a windkessel: relation of arterial and venous windkessels to systemic vascular resistance. AJP Heart 2006, 290:154-162.

[59] Olufsen MS, Peskin CS, Larsen J: Numerical Simulation and Experimental Validation of Blood Flow in Arteries with Structured-Tree Outflow Conditions. Ann Biomed Eng 2000, 28:1281-1299.

[60] Cameron JD, Dart AM: Exercise training increases total systemic arterial compliance in humans. Am J Physiol 1994, 266(H693-H701).

[61] Laskey WK, Parker HG, Ferrari VA, Kussmaul WG, Noordergraaf A: Estimation of total systemic arterial compliance in humans. J Appl Physiol 1990, 69(112-119).

[62] LIU Z, BRIN KP, YIN CFP: Estimation of total arterial compliance: an improved method and evaluation of current methods. Am J Physiol Heart Circ Physiol 1986, 251:H588-H600.

[63] Quick CM, Berger DS, A N: Apparent arterial compliance. AJP Heart 1998, 274(1393-1403).

[64] Stergiopulos N, Meister JJ, Westerhof N: Evaluation of methods for estimation of total arterial compliance. Am J Physiol 1995, 268:H1540-H1548.

[65] Akoi T, Ku DN: Collapse of diseased arteries with eccentric cross section. J Biomech 1993, 26(2):133-142.

[66] Heil M: Stokes flow in collapsible tubes: computation and experiment. J Fluid Mech 1997, 353:285-312.

[67] Ku DN: Blood Flow in Arteries. Ann Rev Fluid Mech 1997, 29:399-434. 
[68] Pedley TJ: Modelling Flow and Oscillations in Collapsible Tubes. Theo Comp Fluid Dyn 1998, 10:277-294.

[69] Downing JM, Ku DN: Effects of Frictional Losses and Pulsatile Flow on the Collapse of Stenotic Arteries. J Biomech Eng 1997, 119:317-324.

[70] Berger SA, Jou LD: Flows in Stenotic Vessels. Annu Rev Fluid Mech 2000, 32:347-382.

[71] Sud VK, Srinivasan RS, Charles JB, Bungo MW: Mathematical modelling of the human cardiovascular system in the presence of stenosis. Phys Med Biol 1993, 38:369-378.

[72] Young DF: Fluid mechanics of arterial of stenoses. J Biomech Eng 1979, 101:439-448.

[73] Pijls N, De Bruyne GJW Band Bech, Liistro F, Heyndrickx GR, Bonnier HJRM, Koolen JJ: Coronary Pressure Measurement to Assess the Hemodynamic Significance of Serial Stenoses Within One Coronary Artery. Circ 2000, 102:2371-2377.

[74] Seely BD, Young DF: Effect of geometry on pressure losses across models of arterial stenoses. J Biomech 1976, 9:439-448.

[75] Schlichting H, Gersten K: Boundary-Layer-Theory. Springer-Verlag Berlin, 8 edition 2003.

[76] White FM: Viscous Fluid Flow. McGraw-Hill International Editions, London 1991.

[77] Lorzhois S, Lagree PY, Marc-Vergnes JP, Cassot F: Maximum Wall Shear Stress in Arterial Stenoses: Application to the Internal Carotid Arteries. J Biomech Eng 2000, 122:1-6.

[78] Provenzano PP, Rutland CJ: A boundary layer model for wall shear stress in arterial stenosis. Biorheol 2002, 39:743-754.

[79] Reese JM, Thompson DD: Shear stress in arterial stenoses: a momentum integral model. J Biomech 1998, 31(11):1051-1057.

[80] Kalse SGC, Bijl H, van Oudheusden BW: A One-Dimensional ViscousInviscid Strong Interaction Model for Flow in Indentet Channels With Seperation an Reattachment. J Biomed Eng 2003, 125:355-362.

[81] Morgan BE, Young DF: An integral method for the analysis of flow in arterial stenoses. Bull Math Biol 1974, 36:39-53. 
[82] Ikeda T, Matsuzaki Y: A One-Dimensional Unsteady Seperable and Reattachable Flow Model for Collapsible Tube-Flow Analysis. $J$ Biomech Eng 1999, 121:153-159.

[83] Veldman AEP: New, Quasi-simultaneous Method to calculate Interacting Boundary Layers. AIAA Journal 1981, 19:79-85.

[84] Veldman AEP: A numerical Method for calculation of Laminar, Incompressible, Boundary Layers With Strong Viscous-Inviscid Interaction. Tech. Rep. 79023U, Dutch National Aerospace Laboratory (NLR) 1979.

[85] He DN: Unsteady Entrance Flow Development in a Straight Tube. $J$ Biomech Eng 1994, 116:355-360.

[86] Smith FT, Purvis R, Dennis SCR, Jones MA, Ovenden NC, Tadjfar M: Fluid flow through various branching tubes. J Eng Math 2003, 47:277-298.

[87] Cave FD, Charlesworth D: Arterial stenosis: an experiment to determine how best to quantify the hydraulic effects of a stenosis. Phys Med Biol 1981, 26(6):1087-1097.

[88] Bhatti MS: Laminar flow in the entrance region of elliptical ducts. $A m$ Soc Mech Eng 1983, 105:290-296.

[89] Theoretical Fluid Mechanics Meeting, 2nd, Albuquerque, NM, June 15-18: Modeling friction factors in non-circular ducts for developing laminar flow., AIAA 1998.

[90] Fleming DP, Sparrow EM: Flow in the Hydrodynamic Entrance Region of Ducts of Arbitrary Cross Section. J Heat Trans 1969, 91:345-354.

[91] Shah RK: A Correlation for laminar Hydrodynamic Entery Length Solutions for Circular and Non-Circular Ducts. J Fluids Eng 1978, 100:177179 .

[92] Sparrow EM, Lin SH: The Developing Laminar Flow and Pressure Drop in the Entrance Region of Annular Ducts. J Basic Eng 1964, 86:827-834.

[93] Yilmaz T: General Equations for Pressure Drop for Laminar Flow in Ducts of Arbitrary Cross Sections. Transactions of the ASME, J Energ Res Tech 1990, 112(220-223).

[94] Lui H, Yamaguchi T: Computer modeling of fluid dynamics related to a myocardial bridge in a coronary artery. Biorheol 1999, :373-390. 
[95] Pedley TJ, Stephanoff KD: Flow along a channel with a time-dependent indentation in one wall: the generation of vorticity waves. J Fluid Mech 1985, 160:337-367.

[96] Ralph WE, Pedley TJ: Flow in a channel with moving indentation. $J$ Fluid Mech 1988, 190:87-112.

[97] Ralph WE, Pedley TJ: Flow in a channel with a time-dependent indentation in one wall. $J$ Biomech Eng 1990, 112:468-475.

[98] Matsuzaki Y, Matsumoto T, Ikeda T, Kitagawa T: Experiments on Steady and Oscillatory Flows at Moderate Reynoldsnumbers in a Quasi TwoDimensional Channel with a Throat. J Biomech Eng 1998, 120:594-601.

[99] Cances E, Gerbeau JF (Eds): Oscillatory flow in a tube with time-dependent wall deformation and its application to myocardial bridges, Volume 14, ESAIM: Proceedings 2005.

[100] Bernhard S, Möhlenkamp S, Tilgner A: Transient integral boundary layer method to calculate the pressure drop in a time dependent vessel geometry applied to myocardial bridges. Biomedical Engineering online 2006.

[101] Bessems D, Rutten MCM, van de Vosse FN: A wave propagation model of blood flow in large vessels using an approximate velocity profile function. 2006. [(submitted)].

[102] Mendez-Nunez LR, Carroll JJ: Comparison of Leapfrog, Smolarkiewicz, and MacCormack Schemes Applied to Nonlinear Equations. Monthly Weather Rev 1993, 121(2):565-578.

[103] Hixon R: Evaluation of a High-Accuracy MacCormack-Type Scheme Using Benchmark Problems. NASA 1997.

[104] Fung YC: Biomechanics Mechanical Properties of Living Tissues. SpringerVerlag, Heidelberg 1981.

[105] Waters SL, Pedley TJ: Oscillatory flow in a tube of time-dependent curvature. Part 1. Perturbation to flow in a stationary curved tube. J Fluid Mech 1999, 383:327-352.

[106] Waters SL, Pedley TJ: Flow in a tube with non-uniform, time-dependent curvature: governing equations and simple examples. J Fluid Mech 1996, 32:237-265. 
[107] Abe H, Hayashi K, Sato M: Data Book on Mechanical Properties of Living Cells, Tissues, and Organs. Springer-Verlag Heidelberg 1996.

[108] KJ G, CJ T: Mechanical Forces: Their Effects on Cells and Tissues. SpringerVerlag Berlin Heidelberg 1997.

[109] Hasegawa H, Kanai H, Hoshimiya N, Koiwa Y: Evaluating the regional elastic modulus of a cylindrical shell with nonuniform wall thickness. J Med Ultrasonics 2004, 31:81-90.

[110] Findley WN, Lai SL, Onaran K: Creep and Relaxation of Nonlinear Viscoelastic Meterials. Dover Publications, Inc. 1989.

[111] Zang L: Die arterielle Sofortdehnung. PhD thesis, Universität Regensburg 1999.

[112] Wootton DM, Ku DN: Fluid Mechanics of Vascular Systems, Diseases, and Thrombosis. Annu Rev Biomed Eng 1999, 1:299-329.

[113] Chaitman BR, Fisher LD, Bourassa MG, K D, Rogers WJ, Maynard C, Tyras DH, Berger RL, Judkins MP, Ringqvist I, Mock MB, Killip T: Effect of coronary bypass surgery on survival patterns in subsets of patients with left main coronary artery disease. Report of the Collaborative Study in Coronary Artery Surgery (CASS). Am J Cardiol 1981, 48(4):765-777.

[114] Gould KL: Quantification of coronary artery stenosis in vivo. Circ Res 1985, 57:341-353.

[115] Gould KL: Pressure-flow characteristics of coronary stenoses in unsedated dogs at rest and during coronary vasodilation. Circ Res 1978, 43:242-253.

[116] Yearwood TL, Misbach GA, Chandran KB: Experimental fluid dynamics of aortic stenosis in a model of the human aorta. Clin Phys Physiol Meas 1989, 10:11-24.

[117] Mates RE, Gupta RL, Bell AC, Klocke FJ: Fluid dynamics of coronary artery stenosis. Circ Res 1978, 42:152-162.

[118] Pontrelli G, Rossoni E: Numerical modelling of the pressure wave propagation in the arterial. Int J Numer Meth Fluids 2003, 43:651-671.

[119] Ge J, Erbel R, Rupprecht HJ, Koch J, Kaerney P, Gorge G, Haude M, Meyer $\mathrm{J}$ : Comparison of intravascular ultrasound and angiography in the assessment of myocardial bridging. Circ 1994, 89:1725-1732. 
[120] Möhlenkamp S, Eggebrecht H, T E, Münzberger S, Schweizer T, Quast B, Erbel R: Muskelbrücken der Koronararterien: mögliche ischämierelevante Normvarianten. Herz 2005, 30:37-47.

[121] Timoshenko SP, Woinowsky-Kriger S: Theory of Plates and Shells. McGrawHill, New York 1959.

[122] Stergiopulos N, Young DF, Rogge TR: Computer simulation of arterial flow with applications to arterial and aortic stenosis. J Biomech 1992, 25:1477-1488.

[123] Segers P, Dubois F, Wachter D, Verdonck P: Role and relevancy of a cardiovascular simulator. J Cardiovasc Eng 1998, 3:48-56.

[124] Podesser BK, Neumann F, Neumann M, Schreiner W, Wollenek G, Mallinger R: Outer Radius-Wall Thickness Ratio, a Postmortem Quantitative Histology in Human Coronary Arteries. Acta Anat 1998, :63-68.

[125] McDonald D: Blood Flow in Arteries. Edward Arnold, second edn edition 1974.

[126] Pedersen EM, Shung HW, Burlson AC, Yoganathan AP: Two-dimensional velocity measurements in a pulsatile flow model of the normal abdominal aorta simulating different hemodynamic conditions. J Biomech 1993, 26(10):1237-1247.

[127] Ludlow DK, Clarkson PA, Bassom AP: New similarity solutions of the unsteady incompressible boundary-layer equations. $J$ Mech appl Math 2000, 53(2):175-206.

[128] Hartree DR: On an equation occurring in Falkner and Skan's approximate treatment of the equations of the boundary layer. Proc Camb Phil Soc 1937, 33:223-239.

[129] Hartmann M: Zeitlich modulierte Statistik der periodisch gestörten turbulenten Kanalströmung. PhD thesis, Universität Göttingen 2001.

[130] Bodley WE: The Non-Linearities of Arterial Blood Flow. Phys Med Biol 1971, 16(4):663-672.

[131] Wibmer M: One-dimensional Simulation of Arterial Blood Flow with Applications. PhD thesis, Technische Universität Wien 2004.

[132] Streeter VL, Keitzer WF, Bohr DF: Pulsatile Blood Flow. New York: McGrawHill 1964. 
[133] Garabedian PR: Partial Differential Equations. New York: John Wiley and Sons 1964.

[134] Raines J, Jaffrin M, Shapiro A: A computer simulation of arterial dynamics in the human leg. J Biomech 1974, 7:77-91.

[135] Press WH, Teukolsky SA, Vetterling WT, Flannery PB: Numerical Recipes in C. Cambridge University Press 1992.

[136] Roache J: Fundamentals of Computational Fluid Dynamics. Hermosa Publishers, 2 edition 1998.

[137] Luo Z, Yang WJ, Stein DP: Errors in the estimate of arterial wall shear rated that result from curve fitting of velocity profiles. $J$ Biomech Eng 1993, 26:383-390.

[138] Dodge JT, Brown BG, Bolson EL, Dodge HT: Intrathoracic Spatial Location of Specified Coronary Segments of the Normal Human Heart. Circ 1988, 78(5):1167-1180.

[139] Dodge JT, Brown BG, Bolson EL, Dodge HT: Lumen Diameter of Normal Human Coronary Arteries. Circ 1992, 86:232-246.

[140] Kumar K: Anatomy of the Human Coronary Arterial Pulsation. J Anat Soc India 2003, 52:24-27.

[141] Fazan VPS, Borges CT, da Silva JH, Caetano AG, Filho OAR: Superficial palmar arch: an arterial diameter study. J Anat 2004, 204:307-311.

[142] Gelberman RH, Panagis JS, Taleisnik J, Baumgaertner M: The arterial anatomy of the human carpus. PartI: The extraosseous vascularity. $J$ Hand Surg 1983, 8:367-375.

[143] Lippert H: Variabilität der Hand- und Fußarterien. Handchir 1984, 16:254-258.

[144] Mezzogiorno A, Passiatore C, Mezzogiorno V: Anatomic Variations of the Deep Palmar Arteries in Man. Acta Anat 1994, 149:221-224.

[145] Yamada T, Gloviczki P, Bower TC, Naessens JM, Carmichael SW: Variations of the Arterial Anatomy of the Foot. Am J Surg 1993, 166:130-135. 


\section{Appendix A}

\section{Cardiovascular system parameters}

$\mathrm{T}$ HE network topology used throughout this study is based on the major 55 arteries mentioned in [20] and a topology of the major 48 coronary arteries presented in [139, 138]. Further the main palmar arteries of the hand and foot were included, whereas the dimensions and exact course are taken from [141, 142, 143, 144, 145]. In figure A.1 we show the arteries of the human cardiovascular system (top left) and the major arteries used in the model (top right). We give the mean transit time to various locations of the vasculature. For example wave reflections caused by the microvasculature in the feet are delayed by about half a period, when they arrive at the heart. The normal anatomic distribution of the coronary arteries (bottom) are based on 83 angiographies presented by Dodge et al. They proposed a terminology, which we used for our model of the coronary arteries. The left coronary arteries (LCA) are essentially composed of the left main coronary artery (LMCA), which bifurcates into the left anterior descending artery (LAD) and the left circumflex artery (LCxA). The main branches of the LAD include the $1^{\text {st }}, 2^{\text {nd }}$ and $3^{\text {rd }}$ diagonal branch (D1, D2, D3) and the $1^{\text {st }}, 2^{\text {nd }}$ and $3^{\text {rd }}$ septal branch (S1, S2, S3). The main branches of the LCxA include the $1^{\text {st }}$ and $2^{\text {nd }}$ obtuse marginal branches (OM1, OM2) and the serial node artery $(\mathrm{SN})$. The single trunk of the right coronary arteries (RCA) branches into the conus branch (CB), the serial node (SN), the right ventricle (RV), the acute marginal $(\mathrm{AM})$, the posterior descending artery $(\mathrm{PD})$ and the atrioventricular node ablation (AVNA). The exact intrathoratic location and course of each one of the 48 arterial segments and branches of the coronary arteries are illustrated at the bottom of the figure. The parameters for each vessel segment can be found in table A.1, the parameters for the windkessel boundary conditions are listed in table A.2. 


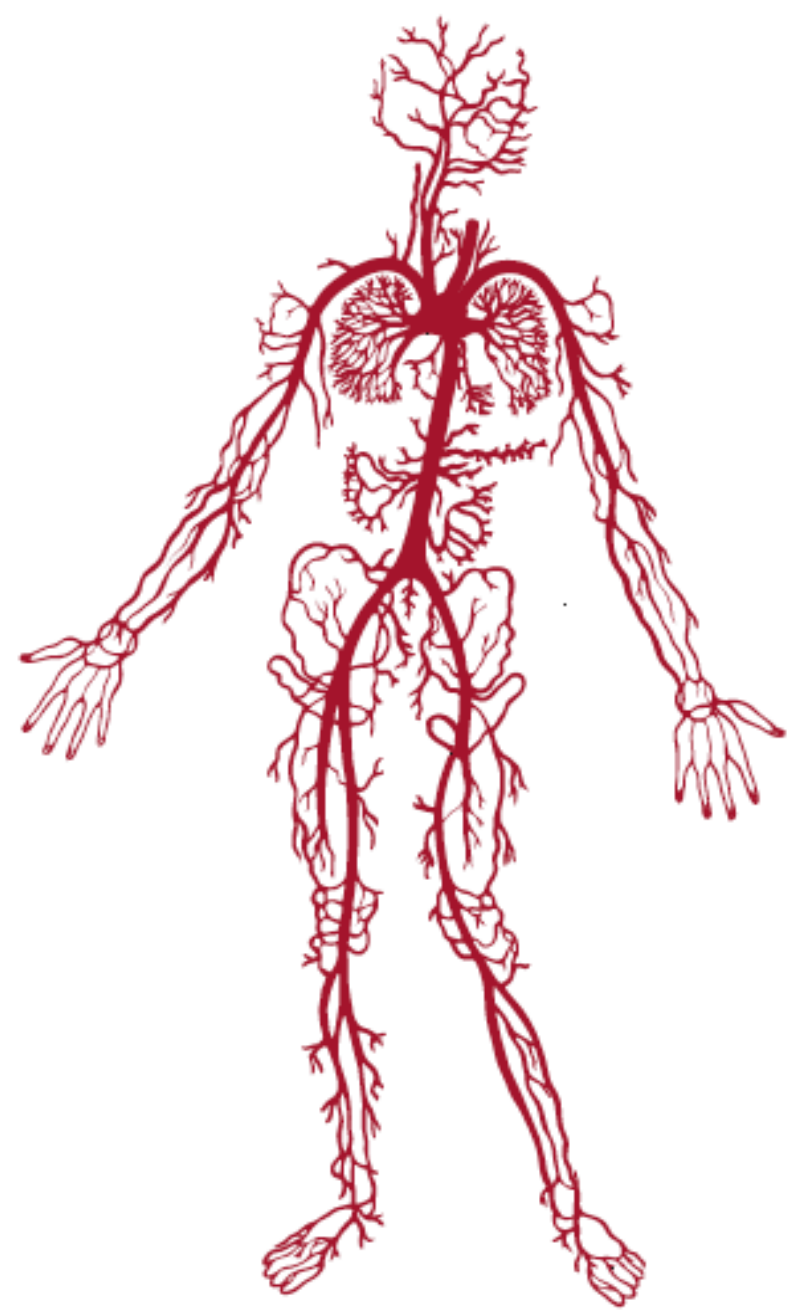

human arterial system

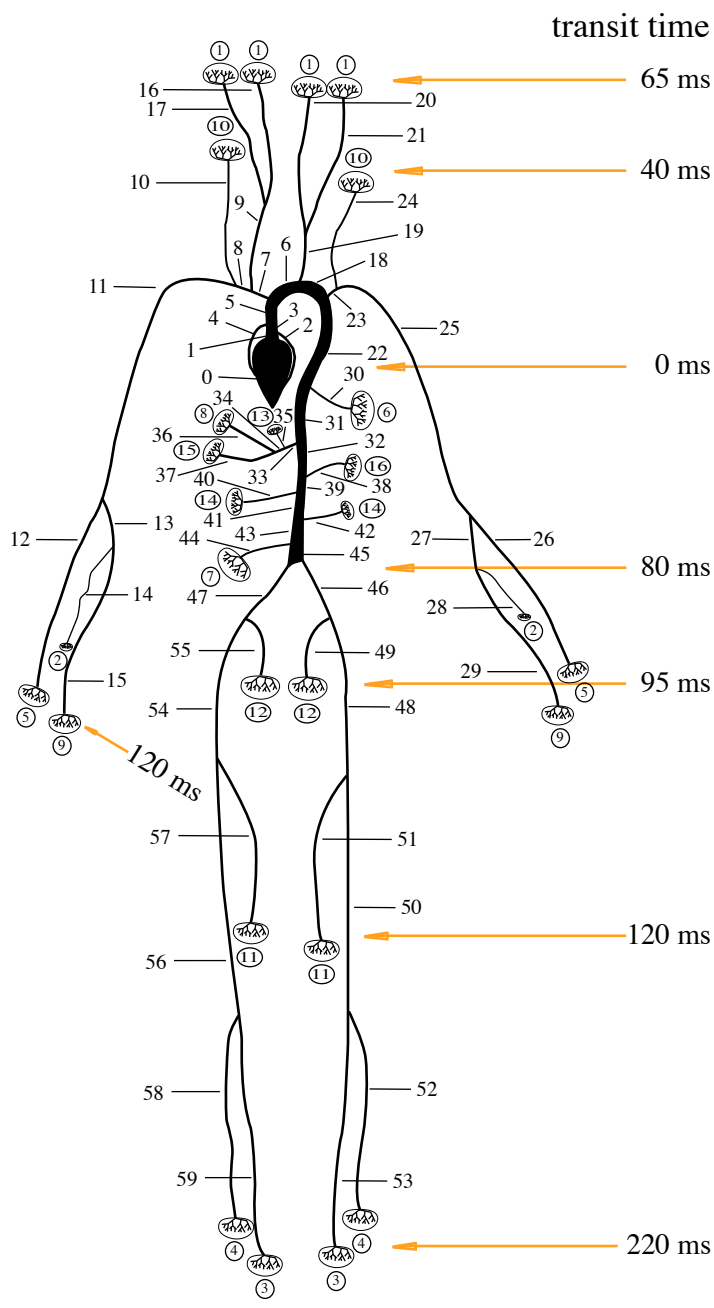

model arterial system
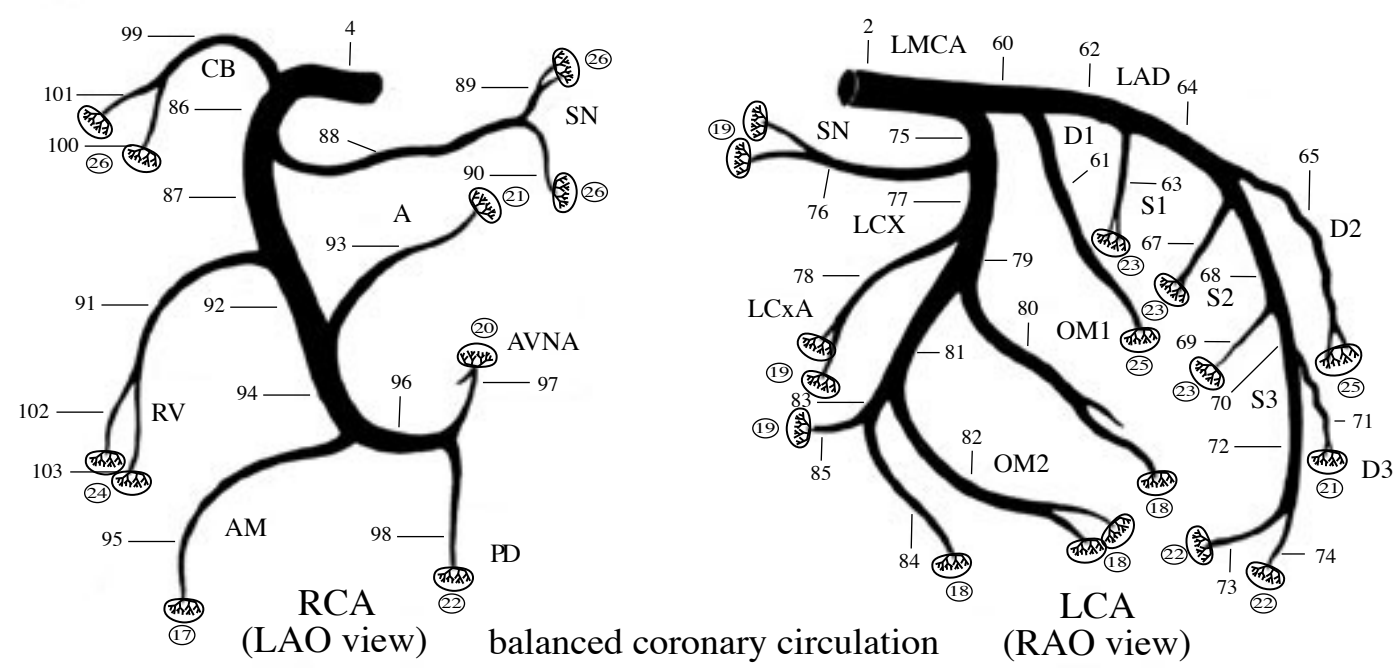

Figure A.1: The illustration shows the major arteries of the human cardiovascular system (top left). The model arterial system (top right) shows the location and exact course of the major arteries given in table A.1 and terminals in table A.2. The topology of the right and left coronary arteries is given on the bottom of the figure. 
Table A.1: Parameters of the major 175 arteries in the human cardiovascular system (compare to figure A.1).

\begin{tabular}{|c|c|c|c|c|c|c|c|}
\hline No. & Name & $\begin{array}{c}R_{0} \\
{[m m]}\end{array}$ & $\begin{array}{c}h_{0} \\
{[\mathrm{~mm}]}\end{array}$ & $\begin{array}{c}l \\
{[\mathrm{~mm}]}\end{array}$ & $\begin{array}{c}E_{0} \\
{[M P a]}\end{array}$ & $\begin{array}{c}c \\
{[m / s]}\end{array}$ & $\begin{array}{c}Z \\
{\left[M P a s / m^{3}\right]}\end{array}$ \\
\hline 0 & left ventricle & 20.00 & 1.98 & 20.0 & 0.87 & 7.39 & 6.21 \\
\hline 1 & ascending aorta I & 14.70 & 1.63 & 10.0 & 0.78 & 7.39 & 11.49 \\
\hline 2 & LMCA & 2.25 & 0.50 & 17.0 & 0.45 & 7.91 & 524.86 \\
\hline 3 & ascending aorta II & 14.40 & 1.61 & 10.0 & 0.77 & 7.39 & 11.97 \\
\hline 4 & $\mathrm{RC} \mathrm{I}$ & 2.0 & 0.46 & 12.0 & 0.47 & 8.28 & 695.43 \\
\hline 5 & ascending aorta III & 14.10 & 1.59 & 20.0 & 0.77 & 7.39 & 12.49 \\
\hline 6 & aortic arch I & 13.53 & 1.55 & 30.0 & 0.75 & 7.39 & 13.56 \\
\hline 7 & brachiocephalic & 6.20 & 0.95 & 35.0 & 0.57 & 7.39 & 64.59 \\
\hline 8 & r subclavian I & 4.23 & 0.75 & 35.0 & 0.49 & 7.40 & 138.87 \\
\hline 9 & r carotid & 3.70 & 0.68 & 177.0 & 0.47 & 7.41 & 181.86 \\
\hline 10 & r vertebral & 1.88 & 0.45 & 148.0 & 0.49 & 8.54 & 811.51 \\
\hline 11 & r subclavian II & 4.03 & 0.72 & 423.0 & 0.48 & 7.40 & 153.07 \\
\hline 12 & $\mathrm{r}$ radial & 1.74 & 0.43 & 235.0 & 0.52 & 8.93 & 990.41 \\
\hline 13 & r ulnar I & 2.15 & 0.49 & 68.0 & 0.45 & 8.04 & 583.97 \\
\hline 14 & $\mathrm{r}$ interosseous & 0.91 & 0.28 & 80.0 & 1.11 & 14.74 & 5978.20 \\
\hline 15 & r ulnar II & 2.03 & 0.47 & 173.0 & 0.46 & 8.23 & 670.53 \\
\hline 16 & $\mathrm{r}$ int carotid & 1.77 & 0.43 & 178.0 & 0.51 & 8.84 & 947.24 \\
\hline 17 & r ext carotid & 1.77 & 0.43 & 178.0 & 0.51 & 8.84 & 947.24 \\
\hline 18 & aortic arch II & 13.0 & 1.51 & 30.0 & 0.74 & 7.39 & 14.69 \\
\hline 19 & 1 carotid & 3.70 & 0.68 & 174.0 & 0.47 & 7.41 & 181.86 \\
\hline 20 & l int carotid & 1.77 & 0.43 & 178.0 & 0.51 & 8.84 & 947.24 \\
\hline 21 & l ext carotid & 1.77 & 0.43 & 178.0 & 0.51 & 8.84 & 947.24 \\
\hline 22 & thoracic aorta I & 11.94 & 1.43 & 60.0 & 0.72 & 7.39 & 17.42 \\
\hline 23 & l subclavian I & 4.23 & 0.75 & 35.0 & 0.49 & 7.40 & 138.87 \\
\hline 24 & 1 vertebral & 1.88 & 0.45 & 148.0 & 0.49 & 8.54 & 811.51 \\
\hline 25 & I subclavian II & 4.03 & 0.72 & 422.0 & 0.48 & 7.40 & 153.07 \\
\hline 26 & 1 radial & 1.74 & 0.43 & 235.0 & 0.52 & 8.93 & 990.41 \\
\hline 27 & l ulnar I & 2.15 & 0.49 & 68.0 & 0.45 & 8.04 & 583.97 \\
\hline 28 & 1 interosseous & 0.91 & 0.28 & 80.0 & 1.11 & 14.74 & 5978.20 \\
\hline 29 & l ulnar II & 2.03 & 0.47 & 173.0 & 0.46 & 8.23 & 670.53 \\
\hline 30 & intercostals & 2.0 & 0.46 & 80.0 & 0.47 & 8.28 & 695.43 \\
\hline 31 & thoracic aorta II & 10.71 & 1.34 & 95.0 & 0.69 & 7.39 & 21.65 \\
\hline 32 & abdomina lI & 8.61 & 1.17 & 53.0 & 0.64 & 7.39 & 33.49 \\
\hline 33 & celiac I & 3.90 & 0.71 & 15.0 & 0.48 & 7.41 & 163.52 \\
\hline
\end{tabular}




\begin{tabular}{|c|c|c|c|c|c|c|c|}
\hline No. & Name & $\begin{array}{c}R_{0} \\
{[m m]}\end{array}$ & $\begin{array}{c}h_{0} \\
{[\mathrm{~mm}]}\end{array}$ & $\begin{array}{c}l \\
{[\mathrm{~mm}]}\end{array}$ & $\begin{array}{c}E_{0} \\
{[M P a]}\end{array}$ & $\begin{array}{c}c \\
{[m / s]}\end{array}$ & $\begin{array}{c}Z \\
{\left[M P a s / m^{3}\right.}\end{array}$ \\
\hline 34 & celiac II & 2.20 & 0.49 & 10.0 & 0.45 & 7.97 & 553.13 \\
\hline 35 & hepatic & 2.20 & 0.49 & 65.0 & 0.45 & 7.97 & 553.13 \\
\hline 36 & gastric & 1.80 & 0.44 & 70.0 & 0.50 & 8.75 & 906.90 \\
\hline 37 & splenic & 2.75 & 0.57 & 63.0 & 0.44 & 7.57 & 335.95 \\
\hline 38 & superior mesenteric & 4.35 & 0.76 & 60.0 & 0.50 & 7.40 & 131.29 \\
\hline 39 & abdominal II & 7.72 & 1.09 & 10.0 & 0.61 & 7.39 & 41.66 \\
\hline 40 & 1 renal & 2.60 & 0.55 & 33.0 & 0.44 & 7.63 & 379.21 \\
\hline 41 & abdominal III & 7.56 & 1.07 & 70.0 & 0.61 & 7.39 & 43.44 \\
\hline 42 & r renal & 2.60 & 0.55 & 33.0 & 0.44 & 7.63 & 379.21 \\
\hline 43 & abdominal IV & 7.40 & 1.06 & 60.0 & 0.60 & 7.39 & 45.34 \\
\hline 44 & inferior mesenteric & 1.60 & 0.40 & 50.0 & 0.56 & 9.44 & 1237.73 \\
\hline 45 & abdominal V & 6.01 & 0.93 & 70.0 & 0.56 & 7.39 & 68.74 \\
\hline 46 & r common illiac & 3.68 & 0.68 & 58.0 & 0.47 & 7.41 & 183.86 \\
\hline 47 & 1 common illiac & 3.68 & 0.68 & 57.0 & 0.47 & 7.41 & 183.86 \\
\hline 48 & l external illiac & 3.47 & 0.66 & 145.0 & 0.46 & 7.43 & 207.15 \\
\hline 49 & l internal illiac & 2.0 & 0.46 & 50.0 & 0.47 & 8.28 & 695.43 \\
\hline 50 & 1 femoral & 2.99 & 0.60 & 443.0 & 0.44 & 7.49 & 281.50 \\
\hline 51 & l deep femoral & 2.55 & 0.54 & 125.0 & 0.44 & 7.66 & 395.69 \\
\hline 52 & 1 tibial posterior & 2.47 & 0.53 & 343.0 & 0.44 & 7.71 & 424.59 \\
\hline 53 & 1 tibial anterior & 1.30 & 0.35 & 330.0 & 0.71 & 11.06 & 2196.77 \\
\hline 54 & r external illiac & 3.47 & 0.66 & 145.0 & 0.46 & 7.43 & 207.15 \\
\hline 55 & $\mathrm{r}$ internal illiac & 2.0 & 0.46 & 50.0 & 0.47 & 8.28 & 695.43 \\
\hline 56 & $\mathrm{r}$ femoral & 2.99 & 0.60 & 443.0 & 0.44 & 7.49 & 281.50 \\
\hline 57 & $\mathrm{r}$ deep femoral & 2.55 & 0.54 & 125.0 & 0.44 & 7.66 & 395.69 \\
\hline 58 & r tibial posterior & 2.47 & 0.53 & 343.0 & 0.44 & 7.71 & 424.59 \\
\hline 59 & r tibial anterior & 1.30 & 0.35 & 330.0 & 0.71 & 11.06 & 2196.77 \\
\hline 60 & LAD I & 1.90 & 0.45 & 8.0 & 0.48 & 8.49 & 790.12 \\
\hline 61 & diagonal artery I & 1.10 & 0.32 & 35.0 & 0.88 & 12.68 & 3517.99 \\
\hline 62 & LAD II & 1.80 & 0.44 & 16.0 & 0.50 & 8.75 & 906.90 \\
\hline 63 & septal artery I & 0.70 & 0.24 & 12.0 & 1.46 & 17.77 & 12177.72 \\
\hline 64 & LAD III & 1.70 & 0.42 & 16.0 & 0.53 & 9.06 & 1052.84 \\
\hline 65 & diagonal artery II & 0.95 & 0.29 & 28.0 & 1.05 & 14.26 & 5305.51 \\
\hline 66 & LAD IV & 1.50 & 0.39 & 5.0 & 0.60 & 9.88 & 1475.35 \\
\hline 67 & septal artery II & 0.55 & 0.21 & 14.0 & 1.78 & 20.51 & 22770.07 \\
\hline 68 & LAD V & 1.20 & 0.34 & 12.0 & 0.78 & 11.80 & 2752.34 \\
\hline 69 & septal artery III & 0.55 & 0.21 & 12.0 & 1.78 & 20.51 & 22770.07 \\
\hline 70 & LAD VI & 1.0 & 0.30 & 6.0 & 0.99 & 13.69 & 4597.96 \\
\hline 71 & diagonal artery III & 0.80 & 0.26 & 12.0 & 1.28 & 16.22 & 8510.53 \\
\hline
\end{tabular}




\begin{tabular}{|c|c|c|c|c|c|c|c|}
\hline No. & Name & $\begin{array}{c}R_{0} \\
{[m m]}\end{array}$ & $\begin{array}{c}h_{0} \\
{[m m]}\end{array}$ & $\begin{array}{c}l \\
{[m m]}\end{array}$ & $\begin{array}{c}E_{0} \\
{[M P a]}\end{array}$ & $\begin{array}{c}c \\
{[m / s]}\end{array}$ & $\begin{array}{c}Z \\
{\left[M P a s / m^{3}\right.}\end{array}$ \\
\hline 72 & LAD VII & 0.85 & 0.27 & 13.0 & 1.19 & 15.52 & 7213.59 \\
\hline 73 & LAD VIII & 0.70 & 0.24 & 10.0 & 1.46 & 17.77 & 12177.72 \\
\hline 74 & diagonal artery IV & 0.65 & 0.23 & 8.0 & 1.56 & 18.62 & 14803.17 \\
\hline 75 & LCX I & 1.80 & 0.44 & 7.0 & 0.50 & 8.75 & 906.90 \\
\hline 76 & Sinuatrial artery & 0.75 & 0.25 & 30.0 & 1.36 & 16.97 & 10130.13 \\
\hline 77 & LCX II & 1.70 & 0.42 & 8.0 & 0.53 & 9.06 & 1052.84 \\
\hline 78 & LACX & 0.65 & 0.23 & 30.0 & 1.56 & 18.62 & 14803.17 \\
\hline 79 & LCX IV & 1.60 & 0.40 & 5.0 & 0.56 & 9.44 & 1237.73 \\
\hline 80 & marginal artery I & 1.05 & 0.31 & 35.0 & 0.93 & 13.17 & 4010.02 \\
\hline 81 & LCX V & 1.30 & 0.35 & 17.0 & 0.71 & 11.06 & 2196.77 \\
\hline 82 & marginal artery II & 1.0 & 0.30 & 32.0 & 0.99 & 13.69 & 4597.96 \\
\hline 83 & LCX VI & 1.0 & 0.30 & 5.0 & 0.99 & 13.69 & 4597.96 \\
\hline 84 & marginal artery III & 0.85 & 0.27 & 22.0 & 1.19 & 15.52 & 7213.59 \\
\hline 85 & LCX VII & 0.70 & 0.24 & 10.0 & 1.46 & 17.77 & 12177.72 \\
\hline 86 & $\mathrm{RC}$ II & 1.90 & 0.45 & 5.0 & 0.48 & 8.49 & 790.12 \\
\hline 87 & RC III & 1.85 & 0.44 & 12.0 & 0.49 & 8.62 & 845.36 \\
\hline 88 & SN I & 0.55 & 0.21 & 30.0 & 1.78 & 20.51 & 22770.07 \\
\hline 89 & SN II & 0.45 & 0.18 & 10.0 & 2.01 & 22.65 & 37568.24 \\
\hline 90 & SN III & 0.40 & 0.17 & 10.0 & 2.13 & 23.83 & 50016.06 \\
\hline 91 & RV I & 1.0 & 0.30 & 25.0 & 0.99 & 13.69 & 4597.96 \\
\hline 92 & RC IV & 1.20 & 0.34 & 10.0 & 0.78 & 11.80 & 2752.34 \\
\hline 93 & A I & 0.80 & 0.26 & 22.0 & 1.28 & 16.22 & 8510.53 \\
\hline 94 & $\mathrm{RC} \mathrm{V}$ & 1.10 & 0.32 & 15.0 & 0.88 & 12.68 & 3517.99 \\
\hline 95 & $\mathrm{AM}$ & 0.65 & 0.23 & 30.0 & 1.56 & 18.62 & 14803.17 \\
\hline 96 & RC VI & 1.0 & 0.30 & 12.0 & 0.99 & 13.69 & 4597.96 \\
\hline 97 & AVNA & 0.40 & 0.17 & 10.0 & 2.13 & 23.83 & 50016.06 \\
\hline 98 & PD I & 0.45 & 0.18 & 13.0 & 2.01 & 22.65 & 37568.24 \\
\hline 99 & CB I & 1.0 & 0.30 & 18.0 & 0.99 & 13.69 & 4597.96 \\
\hline 100 & CB II & 0.50 & 0.19 & 9.0 & 1.89 & 21.55 & 28945.74 \\
\hline 101 & CB III & 0.50 & 0.19 & 7.0 & 1.89 & 21.55 & 28945.74 \\
\hline 102 & RV II & 0.35 & 0.16 & 10.0 & 2.25 & 25.08 & 68756.97 \\
\hline 103 & RV III & 0.35 & 0.16 & 12.0 & 2.25 & 25.08 & 68756.97 \\
\hline 104 & LUA & 1.50 & 0.39 & 40.0 & 0.60 & 9.88 & 1475.35 \\
\hline 105 & LRA & 1.50 & 0.39 & 38.0 & 0.60 & 9.88 & 1475.35 \\
\hline 106 & LSPA I & 1.30 & 0.35 & 20.0 & 0.71 & 11.06 & 2196.77 \\
\hline 107 & LDPA I & 1.20 & 0.34 & 40.0 & 0.78 & 11.80 & 2752.34 \\
\hline 108 & LSPA VI & 1.30 & 0.35 & 20.0 & 0.71 & 11.06 & 2196.77 \\
\hline 109 & LDPA III & 1.20 & 0.34 & 40.0 & 0.78 & 11.80 & 2752.34 \\
\hline
\end{tabular}




\begin{tabular}{|c|c|c|c|c|c|c|c|}
\hline No. & Name & $\begin{array}{c}R_{0} \\
{[m m]}\end{array}$ & $\begin{array}{c}h_{0} \\
{[m m]}\end{array}$ & $\begin{array}{c}l \\
{[\mathrm{~mm}]}\end{array}$ & $\begin{array}{c}E_{0} \\
{[M P a]}\end{array}$ & $\begin{array}{c}c \\
{[m / s]}\end{array}$ & $\begin{array}{c}Z \\
{\left[M P a s / m^{3}\right]}\end{array}$ \\
\hline 110 & LSPA II & 1.0 & 0.30 & 16.0 & 0.99 & 13.69 & 4597.96 \\
\hline 111 & LDIG II & 0.60 & 0.22 & 20.0 & 1.66 & 19.54 & 18224.85 \\
\hline 112 & LDIG I & 0.60 & 0.22 & 25.0 & 1.66 & 19.54 & 18224.85 \\
\hline 113 & LDPA II & 0.80 & 0.26 & 22.0 & 1.28 & 16.22 & 8510.53 \\
\hline 114 & LSPA III & 1.0 & 0.30 & 7.0 & 0.99 & 13.69 & 4597.96 \\
\hline 115 & LDIG III & 0.60 & 0.22 & 20.0 & 1.66 & 19.54 & 18224.85 \\
\hline 116 & LDIG IV & 0.60 & 0.22 & 25.0 & 1.66 & 19.54 & 18224.85 \\
\hline 117 & LDPA IV & 0.80 & 0.26 & 22.0 & 1.28 & 16.22 & 8510.53 \\
\hline 118 & RUA & 1.50 & 0.39 & 40.0 & 0.60 & 9.88 & 1475.35 \\
\hline 119 & RRA & 1.50 & 0.39 & 38.0 & 0.60 & 9.88 & 1475.35 \\
\hline 120 & RSPA I & 1.30 & 0.35 & 20.0 & 0.71 & 11.06 & 2196.77 \\
\hline 121 & RDPA I & 1.20 & 0.34 & 40.0 & 0.78 & 11.80 & 2752.34 \\
\hline 122 & RSPA VI & 1.30 & 0.35 & 20.0 & 0.71 & 11.06 & 2196.77 \\
\hline 123 & RDPA III & 1.20 & 0.34 & 40.0 & 0.78 & 11.80 & 2752.34 \\
\hline 124 & RSPA II & 1.0 & 0.30 & 7.0 & 0.99 & 13.69 & 4597.96 \\
\hline 125 & RDIG II & 0.60 & 0.22 & 20.0 & 1.66 & 19.54 & 18224.85 \\
\hline 126 & RDIG I & 0.60 & 0.22 & 25.0 & 1.66 & 19.54 & 18224.85 \\
\hline 127 & RDPA II & 0.80 & 0.26 & 22.0 & 1.28 & 16.22 & 8510.53 \\
\hline 128 & RSPA III & 1.0 & 0.30 & 7.0 & 0.99 & 13.69 & 4597.96 \\
\hline 129 & RDIG III & 0.60 & 0.22 & 20.0 & 1.66 & 19.54 & 18224.85 \\
\hline 130 & RDIG IV & 0.60 & 0.22 & 25.0 & 1.66 & 19.54 & 18224.85 \\
\hline 131 & RDPA IV & 0.80 & 0.26 & 22.0 & 1.28 & 16.22 & 8510.53 \\
\hline 132 & 1 plantaris lateralis I & 2.20 & 0.49 & 20.0 & 0.45 & 7.97 & 553.13 \\
\hline 133 & 1 dorsalis pedis & 1.30 & 0.35 & 63.0 & 0.71 & 11.06 & 2196.77 \\
\hline 134 & I plantaris lateralis II & 0.80 & 0.26 & 30.0 & 1.28 & 16.22 & 8510.53 \\
\hline 135 & 1 plantaris lateralis III & 1.90 & 0.45 & 40.0 & 0.48 & 8.49 & 790.12 \\
\hline 136 & I plantaris lateralis IV & 1.10 & 0.32 & 15.0 & 0.88 & 12.68 & 3517.99 \\
\hline 137 & 1 metatarseae plantaris I & 1.25 & 0.35 & 10.0 & 0.75 & 11.41 & 2453.09 \\
\hline 138 & 1 metatarseae plantaris II & 1.15 & 0.33 & 10.0 & 0.83 & 12.22 & 3103.53 \\
\hline 139 & I digitales communes I & 0.60 & 0.22 & 15.0 & 1.66 & 19.54 & 18224.85 \\
\hline 140 & 1 metatarseae plantaris III & 1.15 & 0.33 & 10.0 & 0.83 & 12.22 & 3103.53 \\
\hline 141 & 1 digitales communes II & 0.60 & 0.22 & 15.0 & 1.66 & 19.54 & 18224.85 \\
\hline 142 & 1 metatarseae plantaris IV & 1.15 & 0.33 & 10.0 & 0.83 & 12.22 & 3103.53 \\
\hline 143 & 1 digitales communes III & 0.60 & 0.22 & 15.0 & 1.66 & 19.54 & 18224.85 \\
\hline 144 & 1 metatarseae plantaris $\mathrm{V}$ & 1.15 & 0.33 & 12.0 & 0.83 & 12.22 & 3103.53 \\
\hline 145 & I digitales communes IV & 0.60 & 0.22 & 15.0 & 1.66 & 19.54 & 18224.85 \\
\hline 146 & l dorsal metatarsal I & 1.20 & 0.34 & 12.0 & 0.78 & 11.80 & 2752.34 \\
\hline 147 & 1 arcuate I & 1.60 & 0.40 & 10.0 & 0.56 & 9.44 & 1237.73 \\
\hline
\end{tabular}




\begin{tabular}{|c|c|c|c|c|c|c|c|}
\hline No. & Name & $\begin{array}{c}R_{0} \\
{[\mathrm{~mm}]}\end{array}$ & $\begin{array}{c}h_{0} \\
{[\mathrm{~mm}]}\end{array}$ & $\begin{array}{c}l \\
{[\mathrm{~mm}]}\end{array}$ & $\begin{array}{c}E_{0} \\
{[M P a]}\end{array}$ & $\begin{array}{c}c \\
{[\mathrm{~m} / \mathrm{s}]}\end{array}$ & $\begin{array}{c}Z \\
{\left[M P a s / m^{3}\right]}\end{array}$ \\
\hline 148 & 1 arcuate II & 1.40 & 0.37 & 10.0 & 0.65 & 10.42 & 1785.43 \\
\hline 149 & 1 dorsalis pedis I & 0.60 & 0.22 & 15.0 & 1.66 & 19.54 & 18224.85 \\
\hline 150 & 1 arcuate III & 1.20 & 0.34 & 10.0 & 0.78 & 11.80 & 2752.34 \\
\hline 151 & l dorsalis pedis II & 0.60 & 0.22 & 15.0 & 1.66 & 19.54 & 18224.85 \\
\hline 152 & I dorsalis pedis IV & 0.60 & 0.22 & 20.0 & 1.66 & 19.54 & 18224.85 \\
\hline 153 & l dorsalis pedis III & 0.60 & 0.22 & 15.0 & 1.66 & 19.54 & 18224.85 \\
\hline 154 & $\mathrm{r}$ plantaris lateralis I & 2.20 & 0.49 & 20.0 & 0.45 & 7.97 & 553.13 \\
\hline 155 & $\mathrm{r}$ dorsalis pedis & 1.30 & 0.35 & 63.0 & 0.71 & 11.06 & 2196.77 \\
\hline 156 & $\mathrm{r}$ plantaris lateralis II & 0.80 & 0.26 & 30.0 & 1.28 & 16.22 & 8510.53 \\
\hline 157 & $\mathrm{r}$ plantaris lateralis III & 1.20 & 0.34 & 40.0 & 0.78 & 11.80 & 2752.34 \\
\hline 158 & $\mathrm{r}$ plantaris lateralis IV & 0.60 & 0.22 & 15.0 & 1.66 & 19.54 & 18224.85 \\
\hline 159 & r metatarseae plantaris I & 1.25 & 0.35 & 10.0 & 0.75 & 11.41 & 2453.09 \\
\hline 160 & r metatarseae plantaris II & 1.15 & 0.33 & 10.0 & 0.83 & 12.22 & 3103.53 \\
\hline 161 & $\mathrm{r}$ digitales communes I & 0.60 & 0.22 & 15.0 & 1.66 & 19.54 & 18224.85 \\
\hline 162 & $\mathrm{r}$ metatarseae plantaris III & 1.15 & 0.33 & 10.0 & 0.83 & 12.22 & 3103.53 \\
\hline 163 & $\mathrm{r}$ digitales communes II & 0.60 & 0.22 & 15.0 & 1.66 & 19.54 & 18224.85 \\
\hline 164 & $\mathrm{r}$ metatarseae plantaris IV & 1.15 & 0.33 & 10.0 & 0.83 & 12.22 & 3103.53 \\
\hline 165 & $\mathrm{r}$ digitales communes III & 0.60 & 0.22 & 15.0 & 1.66 & 19.54 & 18224.85 \\
\hline 166 & r metatarseae plantaris $\mathrm{V}$ & 1.15 & 0.33 & 12.0 & 0.83 & 12.22 & 3103.53 \\
\hline 167 & $\mathrm{r}$ digitales communes IV & 0.60 & 0.22 & 15.0 & 1.66 & 19.54 & 18224.85 \\
\hline 168 & $\mathrm{r}$ dorsal metatarsal I & 1.20 & 0.34 & 12.0 & 0.78 & 11.80 & 2752.34 \\
\hline 169 & $\mathrm{r}$ arcuate I & 1.60 & 0.40 & 10.0 & 0.56 & 9.44 & 1237.73 \\
\hline 170 & $\mathrm{r}$ arcuate II & 1.40 & 0.37 & 10.0 & 0.65 & 10.42 & 1785.43 \\
\hline 171 & $\mathrm{r}$ dorsalis pedis $\mathrm{I}$ & 0.60 & 0.22 & 15.0 & 1.66 & 19.54 & 18224.85 \\
\hline 172 & $\mathrm{r}$ arcuate III & 1.20 & 0.34 & 10.0 & 0.78 & 11.80 & 2752.34 \\
\hline 173 & $\mathrm{r}$ dorsalis pedis II & 0.60 & 0.22 & 15.0 & 1.66 & 19.54 & 18224.85 \\
\hline 174 & $\mathrm{r}$ dorsalis pedis IV & 0.60 & 0.22 & 20.0 & 1.66 & 19.54 & 18224.85 \\
\hline 175 & $\mathrm{r}$ dorsalis pedis III & 0.60 & 0.22 & 15.0 & 1.66 & 19.54 & 18224.85 \\
\hline
\end{tabular}

$r=$ right, $l=$ left, int $=$ internal, ext = external, Latin numbers denote the segment number. 
Table A.2: Parameters for the windkessel boundary conditions at the terminals of the vascular system (affiliations to terminals in figure A.1).

\begin{tabular}{|c|c|c|c|c|}
\hline No. & group name & affiliations & $\begin{array}{c}R_{t} \\
{\left[k g s^{-1} m^{-4}\right]}\end{array}$ & $\begin{array}{c}C \\
{\left[m^{4} s^{2} / k g\right]}\end{array}$ \\
\hline 1 & carotid & $16,17,20,21$ & $13.90 * 10^{9}$ & $1.34 * 10^{-11}$ \\
\hline 2 & interosseous & 14,28 & $8.43 * 10^{9}$ & $0.22 * 10^{-11}$ \\
\hline 3 & tibial anterior & $\begin{array}{l}149,151,152,153 \\
171,173,174,175\end{array}$ & $5.59 * 10^{9}$ & $3.33 * 10^{-11}$ \\
\hline 4 & tibial posterior & $\begin{array}{l}134,136,139,141 \\
143,145,156,158, \\
161,163,165,167\end{array}$ & $4.77 * 10^{9}$ & $3.90 * 10^{-11}$ \\
\hline 5 & radial & $115,116,129,130$ & $5.28 * 10^{9}$ & $3.52 * 10^{-11}$ \\
\hline 6 & intercostals & 30 & $1.39 * 10^{9}$ & $13.38 * 10^{-11}$ \\
\hline 7 & inferior mesenteric & 44 & $6.88 * 10^{9}$ & $2.70 * 10^{-11}$ \\
\hline 8 & gastric & 36 & $5.41 * 10^{9}$ & $3.44 * 10^{-11}$ \\
\hline 9 & ulner & $111,112,125,126$ & $6.01 * 10^{9}$ & $3.10 * 10^{-11}$ \\
\hline 10 & vertebral & 10,24 & $5.28 * 10^{9}$ & $3.52 * 10^{-11}$ \\
\hline 11 & femoral & 51,57 & $4.77 * 10^{9}$ & $3.90 * 10^{-11}$ \\
\hline 12 & iliac & 49,55 & $7.94 * 10^{9}$ & $2.34 * 10^{-11}$ \\
\hline 13 & hepatic & 35 & $3.63 * 10^{9}$ & $5.13 * 10^{-11}$ \\
\hline 14 & renal & 40,42 & $1.13 * 10^{9}$ & $16.46 * 10^{-11}$ \\
\hline 15 & splenic & 37 & $2.32 * 10^{9}$ & $8.02 * 10^{-11}$ \\
\hline 16 & superior mesenteric & 38 & $0.93 * 10^{11}$ & $20.0 * 10^{-11}$ \\
\hline 17 & $\mathrm{AM}$ & 95 & $1.49 * 10^{11}$ & $1.80 * 10^{-11}$ \\
\hline 18 & $\mathrm{OM}$ & $80,82,84$ & $9.66 * 10^{9}$ & $0.11 * 10^{-11}$ \\
\hline 19 & $\mathrm{SN}$ & $76,78,85$ & $6.9 * 10^{9}$ & $1.40 * 10^{-12}$ \\
\hline 20 & AVNA & 97 & $1.36 * 10^{10}$ & $5.2 * 10^{-13}$ \\
\hline 21 & $\mathrm{D}, \mathrm{A}$ & 71,93 & $3.83 * 10^{10}$ & $2.6 * 10^{-13}$ \\
\hline 22 & LAD, PD & $73,74,98$ & $7.47 * 10^{10}$ & $2.2 * 10^{-13}$ \\
\hline 23 & $\mathrm{~S}$ & $63,67,69$ & $1.0 * 10^{11}$ & $3.3 * 10^{-13}$ \\
\hline 24 & RV & 102,103 & $2.05 * 10^{11}$ & $2.9 * 10^{-14}$ \\
\hline 25 & $\mathrm{D}$ & 61,65 & $1.13 * 10^{10}$ & $7.9 * 10^{-13}$ \\
\hline 26 & $\mathrm{SN}, \mathrm{CB}$ & $89,90,100,101$ & $3.52 * 10^{10}$ & $5.9 * 10^{-14}$ \\
\hline
\end{tabular}




\title{
LEBENSLAUF
}

\author{
Stefan Bernhard
}

\section{Persönliche Angaben}

\author{
Name: $\quad$ Stefan Bernhard \\ Geburtstag: $\quad$ 8. Dezember, 1975 \\ Geburtsort: Darmstadt \\ Nationalität: deutsch \\ Geschlecht: männlich \\ Familienstand: verheiratet mit Regina \\ Kinder: $\quad$ Simon geb. 15.12.2004 \\ Mutter: $\quad$ Brigitte Bernhard geb. Kamleiter \\ Vater: $\quad$ Prof. Dr. Wilfred Bernhard
}

\section{Ausbildung}
1982 - 1985 Grundschule Altbach
1985 - 1986 Grundschule am Gautor Oppenheim
1986 - 1992 Gymnasium zu St. Katharinen Oppenheim
1992 - 1993 Alexander-von-Humboldt Schule Rüsselsheim
1. 8. 1993 Mittlere Reife
1994 - 1998 Berufsbildende Schule I Mainz - Fachoberschule
18. 7. 1997 Industriemechaniker in Geräte- und Feinwerktechnik
25. 6. 1998 Fachhochschulreife in Metalltechnik
1998 - 2000 Studium der Physikalischen Technik an der FH-Wiesbaden
5. 8. 2000 Vordiplom Physikalische Technik (FH)
2000 - 2001 Auslandsstudium University of Central Lancashire UK
1. 6. 2001 Abschluss B.Sc. (Hons) Physics UCLA UK
2001 - 2002 Studium an der Göttingen Graduate School of Physics
15. 11. 2002 Examen Master of Science in Physik
15. 2. 2003 Promotionsstudium in Strömungsmechanik 